\title{
Tierhaltung im mykenischen Reich von Pylos: Die Cn-Serie der Linear B-Texte
}

\section{Inhaltsverzeichnis}

1. Klassifizierung der Tierideogramme................ 7

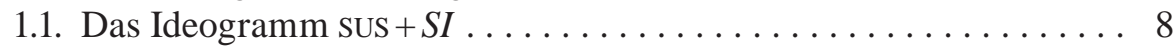

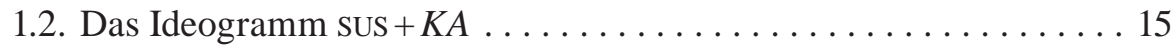

1.3. Kastrierte und unkastrierte männliche Schweine $\ldots \ldots \ldots \ldots 17$

1.4. Kastrierte und unkastrierte männliche Rinder. . . . . . . . 18

1.5. Kastrierte und unkastrierte Tiere in den Linear B-Texten . . . . . . . 20

1.6. Interpretation der Ideogramme sus, BOS, OvIS, CAP und EQU . . . . . 24

1.6.1. Interpretation als Jungtiere? . . . . . . . . . . . . . 24

1.6.2. Interpretation als kastrierte Tiere . . . . . . . . . . . 26

1.6.3. Interpretation als unkastrierte Tiere . . . . . . . . . . . . 27

1.7. Das Ideogramm ovis + TA .................... 36

1.7.1. Interpretation als Mastschafe ................. 36

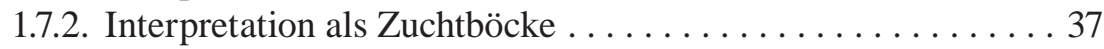

1.7.3. Das Ideogramm CAP $+E \ldots \ldots \ldots \ldots \ldots \ldots \ldots \ldots \ldots \ldots$

1.8. Conclusio . . . . . . . . . . . . . . . . . . . . . . . . . . 39

2. Die mit der Tierhaltung verbundenen Personen $\ldots . \ldots \ldots \ldots \ldots \ldots . . \ldots 0$

2.1. Die pylische Cn-Serie, die „Collectors“ und „Hirten“ sowie der

Vergleich zu den Aufzeichnungen der KN D-, Da-Dv-Serien . . . . 41

2.1.1. Tiere mit den zusätzlichen Angaben pa-ra-jo, wo-ne-we, „Collectors"-Namen und solche ohne nähere Spezifikation. . . 41 2.1.1.1. pa-ra-jo ovis ${ }^{\mathrm{m}} \ldots \ldots \ldots \ldots \ldots \ldots \ldots \ldots \ldots \ldots . \ldots 41$ 2.1.1.2. wo-ne-we ovis ${ }^{\mathrm{m}}$. . . . . . . . . . . . . . . . . 45 2.1.1.3. „Collectors“/,,Besitzer“, „Hirten“ und Herden . . . . . . 47 2.1.1.4. „Hirten“ und Schafe, Ziegen, Schweine in Pylos . . . . . 55

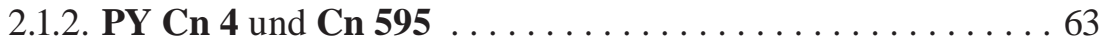

2.1.3. we-re-ke, Schafe und Ziegen $\ldots \ldots \ldots \ldots \ldots \ldots \ldots 64$

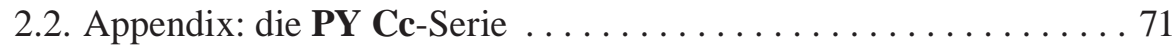

Literaturverzeichnis . . . . . . . . . . . . . . . . . . . . . . . . 71

Die Linear B-Texte der PY Cn-Serie verzeichnen Haustiere unterschiedlichster Art - vorwiegend Schafe, aber auch Ziegen, Schweine und Rinder. ${ }^{1}$ Die diese Tiere kennzeichnenden Ideogramme, welche im ersten Teil der Arbeit ausführlich besprochen werden sollen, sind in den Texten mit verschiedenen Orts- und/oder Personennamen eingetragen. Weiters ist die Einteilung der py-

${ }^{1}$ An dieser Stelle möchte ich meinen Dank an all diejenigen aussprechen, durch deren Hilfe die Verfassung dieses Artikels ermöglicht wurde: Mag. Caroline Dürauer, Mag. Dr. Josef Fischer, Prof. Dr. Sigrid Jalkotzy, Mag. Dr. Georg Nightingale, Prof. Dr. Oswald Panagl, Lic. phil. Günther E. Thüry, Mag. Dr. Jörg Weilhartner; mein besonderer Dank gilt Prof. Dr. John T. Killen für seine hilfreichen und weiterführenden Anmerkungen und Literaturhinweise. 
lischen C-Serie in sechs Untergruppen durch M. Doria ${ }^{2}$ zu nennen, welche als eine Grundlage für die Interpretation der Textinhalte im zweiten Teil des vorliegenden Werkes dient. Ausgehend von dieser Gliederung M. Dorias sind folgende Texte anzuführen, die uns sowohl Auskunft über die vom pylischen Palast gehaltenen Haustierarten als auch einen Einblick in die Organisation der Viehhaltung geben können:

1) Tafeln, die einen Ortsnamen und einen „Hirten“-Namen verzeichnen sowie die Angaben pa-ra-jo beziehungsweise wo-ne-we oder einen „Collector“Namen enthalten können: PY Cn 40, Cn 45, Cn 254 + frr. [+] Cn 272, Cn 599, Cn 600, Cn 643, Cn 655, Cn $719+$ fr. (alle S 4 - Hand 21 bzw. S 719 - H 1); Cn 453 [+] 836, Cn 925 (S 925 - H 1); vermutlich aufgrund der Hand und des Stylus ebenfalls zu dieser Kategorie gehörig: PY Cn 938, Cn 962 (S 4 H 21); Cn 286, Cn $437+$ Xn 490, Cn $485+$ Xn 1076, Cn $570+f r .+$ Xn 1445, Cn 702 [+] Cn 1461, Xn 1059 [+] Cn 1066 + Xn 1472, Cn 1075 (alle S $719-\mathrm{H}$ 1); Cn $491+\mathbf{C n} \mathbf{1 1 6}+$ frr. (S $925-\mathrm{H} 1)^{3}$

2) Tafeln, die den Ausdruck we-re-ke aufweisen: PY Cn 131, Cn 202, Cn 328, Cn $441[+]$ Cn 1069 (alle S 131 - H 1); vermutlich aufgrund der Hand und des Stylus ebenfalls zu dieser Kategorie von Texten gehörig: Cn $285+$ fr., Cn 436 + frr., Cn 491 + Cn 1116 + frr. (alle S 131 - H 1). ${ }^{4}$ Da nach den eindeutig zuzuordnenden Texten der Kategorien 1) und 3) ersichtlich ist, dass der Hand 1 für bestimmte Kategorien von Aufzeichnungen einzelne Styli zugeordnet werden können, lässt sich die Einteilung zu diesen Kategorien wohl auch für die angeführten fragmentarischen Texte vermuten. ${ }^{5}$

3) Tafeln, die das Ideogramm für Schaf (OvIS) mit dem Silbenzeichen TA (= ovis + TA) verzeichnen: PY Cn 4, Cn 595 (beide Texte wurden vom Schreiber 4 - Hand 21 [S 4 - H 21] angefertigt).

4) Die Tafel PY Cn 418, auf der unterschiedliche Tiere pa-ro we-u-da-ne-we aufgelistet sind (Ciii).

5) Die beiden Tafeln PY Cn 3 und Cn 608, welche Mastschweine (sus $+S I$ ) beziehungsweise Rinder (BOs) verzeichnen und beide vom Schreiber 608 Hand 1 gefertigt wurden.

6) Die Tafeln PY Cr 591 und Cr 868 [+] Cr 875, auf denen des Ideogramm für Hirschen CERV angeführt ist.

\footnotetext{
2 Doria 1958.

3 Joins nach Melena 1994-95, 100, 274f., 280f., 283; St. Hiller führte für diese Kategorie Tafeln an, die den Ausdruck a-ko-ra enthalten, s. Hiller-Panagl 1976, 135. Das Wort a-ko-ra alleine ist für die Zuordnung der Texte zur Kategorie 1) jedoch nicht ausreichend. Vielmehr von Bedeutung sind die zusätzlichen Angaben, die neben den Ortsnamen und den „Hirten“Namen erfolgen oder nicht erfolgen.

${ }^{4}$ Joins nach Melena 1994-95, 95, 97, 275, 279f.; neben den aufgezählten Tafeln finden sich noch einige Texte, die aufgrund ihres fragmentarischen Zustandes keiner dieser Kategorien zugeordnet werden können: PY Cn 155, Cn 201 (beide S 155 - Ci); Cn 314, Cn 440 (Ci); Cn 1286 (Ciii). Der Text von PY Cn 1197 wurde ebenfalls vom selben Verfasser (S 131 - H 1) geschrieben und könnte aufgrund der Textstruktur (ON, PN, Tierideogramme) zu den we-re-ke-Tafeln gerechnet werden. Doch fehlt gerade diese Angabe, da die Tafel nach dem Ortsnamen abgebrochen ist und Jörg Weilhartner machte mich noch darauf aufmerksam, dass eine sehr geringe Anzahl von Tieren angeführt ist.

5 Vgl. Palaima 1988, 30 Fn. 37: „Stylus groups often meet the criteria for 'sets' of tablets“; Panagl 1979, 313f: „Typical features proving the 'set' character of a group of tablets are:

- an introductory line concerning the contents of more than one tablet;

- common find spot;

- assignment to a single 'hand';

- corresponding formulas;

- (sometimes also) identical size and format.“
} 
Über die Interpretationen der Aufzeichnungen der pylischen Cn-Serie ist mannigfache Literatur anzuführen, die hier nur auszugsweise vorgestellt werden kann: M. DoRIa Le inscrizioni della classe Cn di Pilo (1958), M. LANG Cnflocks (1966), P. HR. ILIEVSKI A Re-examination of the PY Cn Tablets (1968), ST. HILlER Schafe in Pylos in Hiller-Panagl Die frühgriechischen Texte aus mykenischer Zeit (1976), L. GODART Les collecteurs dans le monde égéen (1992), P. Halstead Lost Sheep? On the Linear B Evidence for breeding Flocks at Mycenaean Knossos and Pylos (1990-91), J. T. KILLEN Records of Sheep and Goats at Mycenaean Knossos and Pylos (1993). ${ }^{6}$

Im Gegensatz zu den Texten der knossischen D-Serien, die ausschließlich Aufzeichnungen über Schafe und deren Wollleistungen enthalten, sind in der pylischen Cn-Serie, wie bereits angeführt auch andere Tierarten (Ziegen, Schweine und Rinder ${ }^{7}$ ) verzeichnet. Ein weiterer Unterschied dieser Serien besteht darin, dass die PY Cn-Serie eine weit geringere Anzahl von Tieren auflistet als dies in den KN D-Serien der Fall ist. ${ }^{8}$ Auch ist in zahlreichen Texten der D-Serien die von den Schafen erwartete Wollmenge durch das Ideogramm LANA (Wolle) angegeben. Ähnliche Eintragungen sind in den pylischen Linear B-Texten, namentlich jenen der $\mathbf{C n}$-Tafeln, nicht vorhanden. Für das Verständnis der Aussagen der pylischen Cn-Serie und deren Unterschiede zu den knossischen D-Serien ist es ratsam sich zunächst allgemein mit den Tierideogrammen und ihrer Interpretation zu beschäftigen. Dabei müssen neben den Ideogrammen der beiden angeführten Serien auch weitere Ideogramme aus den pylischen, knossischen und thebanischen Texten herangezogen werden, welche Tiere repräsentieren.

\section{Klassifizierung der Tierideogramme}

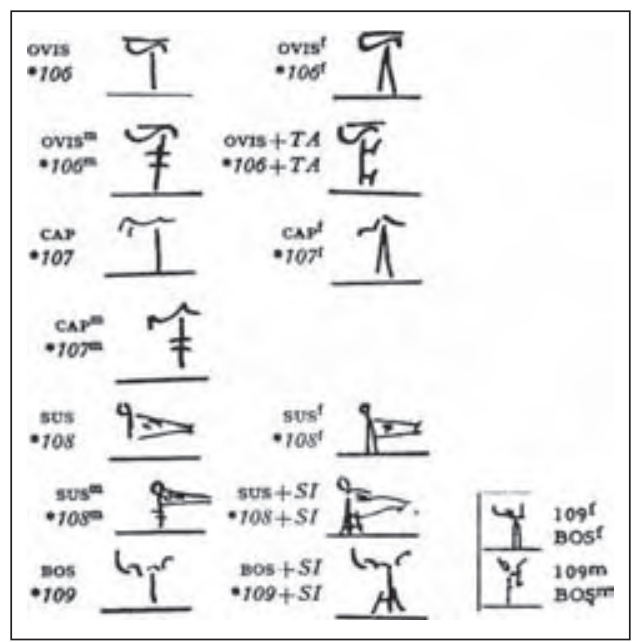

Tab. 1: Tierideogramme (aus Bennett-Olivier 1973, 77; BOS $^{\mathrm{f}}$ und BOS ${ }^{\mathrm{m}}$ aus Bartoněk 2003, 114).

Die Tabelle 1 zeigt die unterschiedlichen Tierideogramme, welche vier wichtige Haustierarten der mykenischen Zeit darstellen. Männliche Tiere wurden durch die Angabe zweier horizontaler Striche gekennzeichnet, weibliche da-

\footnotetext{
${ }^{6}$ Für die ausführliche Literaturangabe s. in der Bibliographie unter den jeweiligen Autoren. 7 Hierbei ist anzumerken, dass das Rinderideogramm jedoch nur in den Texten PY Cn 3 und Cn 418 zu finden ist.

${ }^{8}$ Siehe S. 72 u. Fn. 294.
} 
gegen durch einen zusätzlichen vertikalen Strich. Trotz der als sicher zu geltenden Deutung der Ideogramme $-{ }^{*} 108^{\mathrm{m}}\left(\mathrm{sus}^{\mathrm{m}}\right)$ als männliches und $* 108^{\mathrm{f}}$

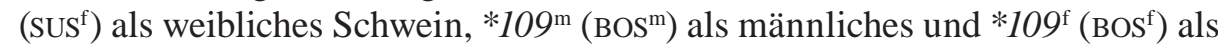
weibliches Rind, ${ }^{*} 106^{\mathrm{m}}\left(\right.$ OVIS $\left.^{\mathrm{m}}\right)$ als männliches und $* 106^{\mathrm{f}}\left(\right.$ OVIS $\left.^{\mathrm{f}}\right)$ als weibliches Schaf, ${ }^{*} 107^{\mathrm{m}}\left(\mathrm{CAP}^{\mathrm{m}}\right)$ als männliche und ${ }^{*} 107^{\mathrm{f}}\left(\mathrm{CAP}^{\mathrm{f}}\right)$ als weibliche Ziege - sind doch immer noch einige Fragen offen:

- Welchen Geschlechts waren die Schweine und Rinder, deren Ideogramme mit der Ligatur SI näher definiert wurden?

- Welchen Geschlechts waren die Tiere, deren Ideogramme ohne zusätzliche Kennzeichnung - zwei horizontalen für Strichen für männlich oder einem zweiten vertikalen Strich für weiblich - also sus, BOS, OVIS, CAP - geschrieben wurden?

- Wie sind die als ovis + TA bezeichneten Schafe zu deuten?

In den folgenden Kapiteln soll daher ausführlich auf die Interpretation dieser drei Fragen eingegangen werden.

\subsection{DAS IDEOGRAMM SUS $+S I$}

Beginnen wir zunächst mit den als sus $+S I$ bezeichneten Schweinen. Das mit der Ligatur SI versehene Ideogramm sus ist auf den Tafeln PY Cn 608, Ua 25.1, Un 2.6, Un 138.4 sowie auf den thebanischen Tonplomben TH Wu 52.a und Wu 68. $\alpha$ zu finden. Keine Hinweise sind auf den Linear B-Texten von Knossos enthalten, obwohl die Ideogramme sus (Schwein), sus ${ }^{f}$ (weibliches Schwein) und sus ${ }^{\mathrm{m}}$ (männliches Schwein) vor allem in der knossischen CoSerie vorkommen.

PY

\begin{tabular}{|c|c|c|c|}
\hline \multirow[t]{11}{*}{ Cn 608} & .1 & \multicolumn{2}{|c|}{ jo-a-se-so-si , si-a } \\
\hline & .2 & o-pi-da-mi-jo & \\
\hline & 3 & pi-*82 & sus+SI 3 \\
\hline & .4 & me-ta-pa & sus $+S I 3$ \\
\hline & .5 & pe-to-no & sus $+S I 6$ \\
\hline & .6 & pa-ki-ja-si & sus+SI 2 \\
\hline & .7 & a-pu $u_{2}$-we & sus $+S I 2$ \\
\hline & .8 & a-ke-re-wa & $\mathrm{sus}+S I 2$ \\
\hline & .9 & e-ra-te-i & sus $+S I$ \\
\hline & .10 & ka-ra-do-ro & $\mathrm{sus}+S I 2$ \\
\hline & .11 & ri-jo & sUS+SI 2 \\
\hline
\end{tabular}

PY

Un 2 .1 pa-ki-ja-si , mu-jo-me-no, e-pi , wa-na-ka-te , S2 - H1 a-pi-e-ke, o-pi-te-ke-e-u

HORD 16 T 4 CYP $+P A$ T 1 v 3 o v 5

FAR 1 T 2 OLIV 3 T $2 * 132$ s $2 M E$ s 1

NI 1 BOS 1 ovis $^{\mathrm{m}} 26$ ovIs $^{t} 6 \mathrm{CAP}^{\mathrm{m}} 2 \mathrm{CAP}^{t} 2$

sus $+S I 1$ sus $^{t} 6$ vin 20 s $1 * 1462$

PY

Un 138 .1 pu-ro, qe-te-a, , pa-ro, du-ni-jo

$\mathrm{S} 138-\mathrm{H} 42$ HORD 18 T 5 pO-qa OLIV 4 T 3 v 5

VIN 13 ovIs $^{\mathrm{Im}} 15$ WE 8 ovIs' $^{\prime} 1$ CAP $^{\mathrm{mm}} 13$ sUS 12

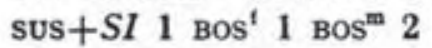

me-za-wo-ni HORD 4 T 8 v 1 ka-pa oliv 7 
PY
Ua $17 \quad .1$
]1 T 7 VIN 47 S 1 v 5
17 ovis $^{t} 7$ WE 17 CAP $^{\text {m }} 31$ sus $^{t} 20$
$\mathrm{S} 138-\mathrm{H} 42$
17 lat.inf.
]14 [
$17 \mathrm{v}$.
PY
$\begin{array}{ccccc}\text { Ua } 25 & .1 & \text { sus }+ \text { SI } 3 & \text { BOS }^{t} 2 & \text { BOS }^{\mathrm{m}} 8 \\ & .2 & \text { ovIs }^{\mathrm{m}} 67 & \end{array}$
25 v. HORD 29 T 8 v 2
$\mathrm{TH}$

$52(W u)$

$\begin{array}{ll}. \alpha & \text { SUS+SI } \\ \cdot \beta & a-k o-r a-j o \\ . \gamma & \text { vacat }\end{array}$

$\mathrm{TH}$

68 (Wu) supra sigillum $\mathbf{F}[5]$

(8)

$\begin{array}{lll}. \alpha & \text { SUS+SI } & \text { supra sigillum } F[5] \\ . \beta & a-k o-r a-j o & \\ . \gamma & \text { vacat } & \end{array}$

Das Ideogramm sus der angeführten Texte ist jeweils mit dem Zeichen SI determiniert. Dieses SI wird als die verkürzte Form des auf PY Cn 608.1 ebenfalls verzeichneten Wortes si- $a_{2}$-ro angesehen, welches wiederum mit dem klassisch-griechischen Wort $\sigma 1 \alpha \lambda$ ó $\omega$ in Zusammenhang gebracht wird, das „mäs-

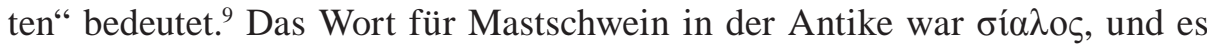

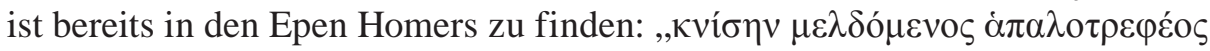

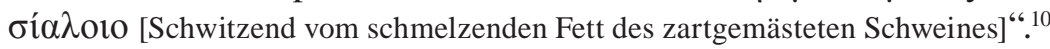

M. Ventris und J. CHADWICK interpretierten in Documents in Mycenaean Greek die Tafel PY Cn 608, auf Grundlage der Einleitungsworte jo-a-se-so-si si- $a_{2}$-ro o-pi-da-mi-jo („How the local inhabitants will fatten fat hogs“) der ersten beiden Zeilen, als einen Vorschreibungstext. ${ }^{11}$ Der Palast von Pylos erteilte demnach an die Bewohner verschiedener Gemeinden (o-pi-da-mi-jo) den Auftrag zum Mästen von Schweinen. Die auf der Tafel genannten Orte befinden sich allesamt in der Diesseitigen Provinz (de-we-ro- $\left.a_{3}-k o-r a-i-j a\right)$ des pylischen Reiches. ${ }^{12}$ Die Anzahl der zu mästenden Schweine variiert von 6 Stück in pe-to-no (Zeile .5), je 3 in pi-*82 (Zeile .3), me-ta-pa (Zeile .4) und e-ra-to

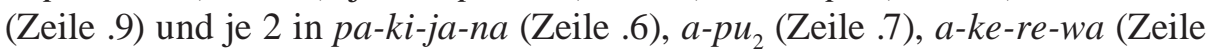
.8), ka-ra-do-ro (Zeile .10) und ri-jo (Zeile .11). Dabei scheint die Anzahl der Mastschweine in einer direkten Relation zur Leistungskapazität der einzelnen Ortschaften gestanden zu haben. So erscheinen dieselben Ortsnamen auf den Tafeln PY Vn 19, Vn 20 und Texten der pylischen Ma-Serie (s. Tab. 2), auf denen die Angaben über die Mengenverhältnisse verschiedener Produkte adäquat zu jenen von PY Cn 608 sind. ${ }^{13}$

\footnotetext{
${ }^{9}$ Ventris-Chadwick 1973, 582; Aura Jorro 1993, s.v. si- $a_{2}-$ ro.

${ }^{10}$ Hom.Il.21,363.

11 Ventris-Chadwick 1973, $205 f$.

12 Dickinson 1994, 82.

13 Ventris-Chadwick 1973, 142f., 415f.; Bennet 1998, 117ff., 120 Tab. 1; Bennett-Olivier 1973, $192 f$.
} 


\begin{tabular}{|llllllll|}
\hline No. & Ma & Name & $\bullet 146$ & ka-ke-we & Vn 20 & Cn 608 & Vn 19 \\
1 & 225 & pi॰82 & 28 & 1 & 50 & 3 & $?$ \\
2 & 90 & me-ta-pa & 28 & 1 & 50 & 3 & $?$ \\
3 & 120 & pe-to-no & 63 & 2 & 100 & 6 & 200 \\
4 & 221 & pa-ki-ja-pi & 22 & 1 & 35 & 2 & 80[ \\
5 & 124 & a-pu, $-w e$ & 23 & 1 & 35 & 2 & 60 \\
6 & 222 & a-ke-re-wa & 23 & & 30 & 2 & 40 \\
$7 \mathrm{~A}$ & & & & & 50 & 3 & $?$ \\
8 & 346 & ka-ra-do-ro & 17 & 1 & 40 & 2 & $?$ \\
9 & 193 & ri-jo & 18 & & 20 & 2 & $?$ \\
\hline
\end{tabular}

Tab. 2: Verhältnis der Angaben der PY Ma-Serie zu PY Cn 608, Vn 19 und Vn 20 (nach Bennett-Olivier 1973, 192).

Bevor mit einer eingehenden Interpretation des Ideogrammes sus + SI und den damit bezeichneten Mastschweinen begonnen wird, sollen kurz noch die weiteren Texte vorgestellt werden, in denen das Zeichen sus + SI enthalten ist.

Beginnen wir zunächst mit jenen der pylischen U-Serien. Die Tafel PY Un 2 verzeichnet Abgaben verschiedenster Art. J. CHADWICK interpretierte die ersten beiden Zeilen des Textes pa-ki-ja-si mu-jo-me-no e-pi wa-na-ka-te a-pi$e$-ke o-pi-te-ke-e-u als „on the occasion of the king's initiation at Pakijana“. ${ }^{14}$ Dieser Deutung folgten J. T. KILLEN ${ }^{15}$ sowie Chr. Piteros, J.-P. Olivier und J. L. MelenA: „à pa-ki-ja-na, à l'occasion de l’initiation du wanax, le ,préposé aux teukhea' a rassemblé“' ${ }^{16}$ Benötigt wurden die auf Un 2 angeführten Tiere, der Honig $(M E)$, die Kommodität *146 und die pflanzlichen Produkte also für eine Feierlichkeit, die in Verbindung mit dem wanaks von Pylos zu sehen ist. Unter den im Text aufgelisteten Tieren befindet sich ein Mastschwein (Zeile .6), wodurch sich zeigt, dass dieses eindeutig für eine festliche Gelegenheit bestimmt war.

Wiederum ein Mastschwein ist auf der Tafel PY Un 138 in Zeile .4 verzeichnet. Dieser Text enthält in der Zeile 1 die Worte pu-ro qe-te- $a_{2}$ pa-ro du-ni-jo, in den Zeilen .2-.4 folgt die Auflistung pflanzlicher Produkte (Getreide, Oliven, Wein) und verschiedener Tiere (weibliche, männliche und junge Schafe, männliche Ziegen, Schweine, ein Mastschwein, ein weibliches und ein männliches Rind). Die Zeile .5 beginnt mit einem Personennamen im Dativ, me-za-wo-ni, auf den die Ideogramme HORD und ka-pa OLIV folgen. ${ }^{17}$ Der in Zeile .1 genannte Mann namens du-ni-jo wird als der für die Aufbewahrung oder Lieferung der Kommoditäten verantwortliche Palastfunktionär gedeutet. ${ }^{18} \mathrm{R}$. PALMER interpretierte die in derselben Zeile geschriebenen Worte puro qe-te- $a_{2}$ als „paid by the palace“ ${ }^{19}$ Die Güter waren demnach von oder bei dem Palastfunktionär du-ni-jo (pa-ro du-ni-jo), der für die Aufstellung und Verwaltung derselben zuständig gewesen sein musste. Auch R. PALMER sprach sich dafür aus, den Namen me-za-wo-ni ebenfalls mit dem in der Zeile .1 angeführten Wort pa-ro zu verbinden. ${ }^{20}$ Und TH. PALAima ging ebenfalls davon aus, dass sich die Angabe qe-te- $a_{2}$ pa-ro auf den Namen me-za-wo-ni bezieht. ${ }^{21}$

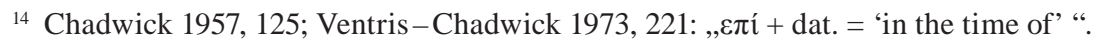

${ }^{15}$ Killen 1999, 332; Killen 1979, $169 f$.

${ }^{16}$ Piteros-Olivier-Melena 1990, 179.

${ }_{17}$ Zur Interpretation von me-za-wo-ni s. Olivier 1960, 118.

${ }_{18}$ Lejeune 1957, 130, 136; Lindgren 1973 II, 41; Hutton 1990-91, 119; R. Palmer 1994, 96f.; Palaima 2000, 266.

${ }_{19}$ R. Palmer 1994, 96; eine ausführliche Diskussion zu qe-te-º s. bei Hutton 1990-91, $105 \mathrm{ff}$.

${ }^{20}$ R. Palmer 1994, 97; vgl. Ventris-Chadwick 1973, 220.

${ }^{21}$ Palaima 2000, 267.
} 
Dies bedeutet, dass das in der Zeile .5 genannte Getreide und die Oliven von oder bei *me-za-wo waren.

Der Palast selbst war nach der Angabe pu-ro qe-te- $a_{2}$ anscheinend der Verpflichtete, die beiden Männer du-ni-jo und *me-za-wo dagegen mit der Verwaltung der palatialen Güter betraut. Nachdem kein Empfänger genannt wird, ist zu vermuten, dass der Palast selbst als solcher zu sehen ist. Es handelt sich bei der Aufzeichnung von PY Un 138 vermutlich um die Niederschrift einer ,palastinternen“ Transaktion. Die pflanzlichen Produkte und Tiere sollten demnach aus der Verwahrung und Verwaltung der beiden Palastfunktionäre ${ }^{22}$ zur tatsächlichen Verwendung an den Palast übergeben werden. Wir können wohl davon ausgehen, dass die meisten Tiere nicht im Palastareal selbst, sondern außerhalb desselben in Palastställen oder auf Palastweiden gehalten wurden. ${ }^{23}$ Dass auf Un 138 keine Angabe von Hirtennamen erfolgt, scheint darauf hinzuweisen, dass sie sich bereits unter der Verwaltung des du-ni-jo befanden. Sie waren zusammen mit den pflanzlichen Produkten (unter du-ni-jo und *me$z a-w o$ ) wohl bereits für eine spätere Verwendung durch den Palast vorgesehen. Der Vorgang der Lieferung lässt sich auf den thebanischen Tonplomben feststellen. ${ }^{24}$ TH Wu 51. $\beta . \gamma$, Wu 65. $\beta . \gamma$ und Wu 96. $\beta . \gamma$ verzeichnen die Worte te-qa-de qe-te- $a_{2}$. Der Allativ te-qa-de (nach Theben) zeigt, dass die auf den Seiten.$\alpha$ angeführten Tiere (sus ${ }^{\mathrm{m}}$, sus ${ }^{\mathrm{f}}$, ovIs ${ }^{\mathrm{f}}$ ) an den thebanischen Palast gesandt wurden. Die Nennung von te-qa-de scheint vor allem in in Zusammenhang mit dem Begriff qe-te- $a_{2}$ zu stehen. So zeigt sich mit dieser Angabe auf Wu 51, Wu 65 und Wu 96 der Vorgang der Lieferung von Tieren, welche aus dem Bestand des Palastes von Theben stammten, wohl in Verwahrung und unter der Verwaltung eines Palastfunktionärs ${ }^{25}$ gewesen waren und nun nach Theben geliefert wurden (,paid by the palace ${ }^{\text {“26) }}$. Die Angabe von te-qa-de auf den thebanischen Tonplomben und jene von pu-ro auf PY Un 138 zeigt somit, dass sich die Tiere bis auf Abruf an einem Platz außerhalb der Paläste befunden haben.

Der Palast von Pylos ist also derjenige, aus dessen Bestand die Tiere und pflanzlichen Güter, welche auf Un 138 verzeichnet sind, genommen wurden. Eine Frage, die sich in Bezug auf die Lieferung der Kommoditäten von Un 138 stellt ist jedoch, wozu sie vom Palast von Pylos benötigt wurden. J. T. KILLEN sah auf der Tafel Un 138 Güter verzeichnet, die für ein „ceremonial

${ }^{22}$ Es könnte sich bei *me-za-wo ebenfalls um einen Palastfunktionär, vergleichbar zu du-nijo, gehandelt haben. Zumindest dürfte er ein Verwaltungsbeamter des Palastes von Pylos gewesen sein. Die Verwaltung von pflanzlichen Produkten und Tieren durch du-ni-jo und ausschließlich pflanzlicher Güter durch *me-za-wo scheint nicht verwunderlich, wenn man bedenkt, dass $d u$-ni-jo in Texten unterschiedlichsten Inhaltes zu finden ist, die sich sowohl auf Tiere als auch auf Männer in bestimmten Funktionen (PY Ae 8, Ae 72, Ae 264), Landbesitz (Ea 811, Ea 59.7) und Getreide beziehen (Fn 79.3), während der Name me-za-wo-ni vielleicht in der Form me-za-ne nur auf einem einzigen weiteren Text (PY Fn 50.4) mit Getreide (HORD) in Verbindung zu bringen ist. Siehe dazu Lindgren 1973 I, 43f., 79; Lindgren 1973 II, 40f., 53, 187; Palaima 1989, 110; Olivier 1960, 118. Es ist durchaus in Betracht zu ziehen, dass die Aufgaben des du-ni-jo ein weit größeres Spektrum umfassten als jene des *me-za-wo und sich dieser Umstand auch in der Tafel Un 138 widerspiegelte. Zu bedenken ist eine mögliche Einschränkung der Belange des *me-za-wo aufgrund der Textfunde. So konnte dieser Mann genauso mehrere Tätigkeiten im Rahmen der Palastverwaltung erfüllt haben, doch sind Aufzeichnungen darüber nicht erhalten.

${ }^{23}$ Vgl. Piteros-Olivier-Melena 1990, $183 f$.

${ }^{24}$ Vgl. Piteros-Olivier-Melena 1990, 172, 175, $182 \mathrm{f}$.

${ }^{25}$ Der Siegelbenutzer des Siegels mit dem Abdruck Typ D wurde von Panagiotopoulos 2002, 225 als Beamter beschrieben.

${ }^{26}$ R. Palmer 1994, 96. 
banquet" gedacht waren und TH. PALAIMA deutete dieselben Kommoditäten als „to be paid as a religious obligation“.$^{27}$ Nicht zu leugnen ist, dass die Zusammenstellung der Tiere, vor allem des Mastschweins (suS $+S I)^{28}$ und der mit dem Ideogramm sus definierten Schweine, ${ }^{29}$ sowie der pflanzlichen Güter auf die Nutzung bei einem Festmahl hinweist.

Die nächste Tafel, welche das Ideogramm sus + SI enthält, ist PY Ua 25. Der Text listet drei Mastschweine, zwei weibliche und drei männliche Rinder sowie 67 männliche Schafe auf. Weitere Informationen sind nicht vorhanden. Die Angaben von Ua 25 dürfen wohl ebenfalls als Ablieferungen von pflanzlichen Gütern und Tieren gesehen werden, die vom pylischen Palast zur Verfügung gestellt wurden. Zumindest lässt die fehlende Orts- beziehungsweise Personenangabe auf der Tafel diesen Schluss zu. Auch ist zu bedenken, dass der Text von Ua 25 von derselben Schreiberhand (S 138 - H 42) verfasst wurde, wie jener der Tafel PY Un 138.

Weitere Mastschweine sind auf den Tonplomben aus Theben verzeichnet. Das Ideogramm suS +SI ist auf TH Wu 52. $\alpha$ und Wu 68. $\alpha$ vertreten, beide Male wurde zusätzlich der Terminus a-ko-ra-jo angegeben (Wu 52. $\beta$, Wu 68. $\beta$ ). Dieses Wort findet sich auch in den Texten der knossischen Co-Serie - a-ko-ra-jo auf KN Co 907.1 und als a-ko-ra-ja auf Co 903.1, Co 904.1, Co 906.1, Co 909.1 und Co 910.1; die Form a-ko-ra dagegen ist in Pylos auf den Tafeln PY Cn 655.5.6.11.12.13, Cn 453 [+] 836.1 vorhanden. Als generell anerkannt darf gelten, dass der Begriff a-ke-re mit dem klassisch-griechischen

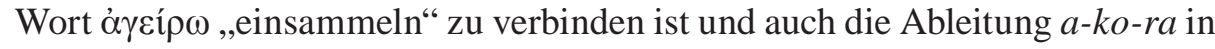
diesem Sinne verstanden werden darf. ${ }^{30}$ Eine ausführliche Darlegung der Bedeutung dieser Wörter würde in diesem Artikel jedoch zu weit führen. Wichtig ist in Zusammenhang mit der Interpretation von Mastschweinen, dass nach V. Aravantinos die Tonplomben aus Theben Tiere anzeigen, die für den Palast bestimmt waren. ${ }^{31}$

Vordringlich stellt sich die Frage nach dem Geschlecht der als sus +SI definierten Schweine. Der in Documents in Mycenaean Greek verwendete Begriff „hog“32 bezeichnet ganz allgemein das Hausschwein, das kastrierte Mastschwein, das Schlachtschwein, im Amerikanischen aber auch den Eber. Ein Mastschwein ist per definitionem generell jedes Schwein, das primär zur Schlachtung gedacht ist („Mästung, spezielle Fütterung von Schlachtvieh zur Erhöhung der Fett- u. Fleischmasse. Man unterscheidet die Eiweiß-M. bei jungen Tieren zur Steigerung des Fleischgewichtes u. die Fett-M. bei älteren Tieren.“33). In den folgenden Abschnitten sind drei Fragen in Bezug auf die Mastschweine zu klären: sind hier kastrierte männliche Schweine oder Eber verzeichnet? Warum wird die Mast auf der Tafel PY Cn 608 mit dem Wort $s i-a_{2}$-ro nochmals betont? Welches Alter ist für die zu mästenden Schweine vorauszusetzen?

${ }^{27}$ Killen 1992, 369; Th. Palaima 2000, 262 u. Fn. 2, S. 266; s. auch Piteros-Olivier-Melena 1990, 181; Hutton 1990-91, 118f., 124, 126, 128.

28 Siehe S. 12.

${ }^{29}$ Siehe S. 27ff.

${ }^{30}$ Ventris-Chadwick 1973, 200, 434, 529; Ilievski 1968, 618; Carlier 1992, 160; Bennet 1992, 68.

31 Aravantinos 1990, 162; allerdings ging er nicht unbedingt vom Gebrauch aller Tiere für den Kult aus; so schrieb V. Aravantinos, dass das auf den Tonplomben vorkommende Vieh zum Verzehr durch das Palastpersonal oder die Arbeitergruppen, aber auch für religiöse Zwecke gedacht sein könnte. Im Falle der sus + SI plädiere ich doch für letzteres; s. S. 12.

32 Ventris-Chadwick 1973, 205.

${ }^{33}$ Definition von Mast nach Bertelsmann Lexikon 1973, 299. 
Obwohl das Ideogramm für Schwein ohne Geschlechtsdefinition angegeben wurde (sus mit der Ligatur SI versehen), sollten bei fett gemästeten Schweinen weibliche Tiere von vornherein ausgeschlossen sein. ${ }^{34}$ Sauen werden und wurden zwar auch geschlachtet, dienten jedoch vorrangig zur Zucht. Eine fett gemästete Sau ist nicht mehr, oder nur unter Schwierigkeiten in der Lage für Nachwuchs zu sorgen. Eine zu starke Verfettung während der Trächtigkeit führt zu Geburtsstörungen und in Folge zu Problemen der Gesundheit und Fruchtbarkeit. ${ }^{35}$ Bereits der römische Schriftsteller Plinius d. Ä. schrieb darüber: „Allzu fetten Schweinen fehlt es an Milch, auch ist ihr erster Wurf weniger zahlreich““ ${ }^{36}$ Nicht nur der erste Wurf, sondern generell die Fruchtbarkeit einer Sau wird durch zuviel Fett negativ beeinflusst. Jedoch ist auch die Mast eines weiblichen Schweins möglich, wenn man es nicht zur Zucht verwenden möchte. Auskunft über die Mästung von Sauen gibt uns wiederum Plinius d. Ä., indem er ein grausames Verfahren beschreibt: „Man kastriert auch die Sauen, wie die Kamele, indem man sie nach zweitägigem Fasten an den Vorderbeinen aufhängt und die Gebärmutter herausschneidet; so werden sie schneller fett““ ${ }^{37}$ Der römische Agrarschriftsteller Columella berichtet ebenfalls über eine derartige Prozedur: „Auch den Säuen schabt man die Gebärmutter aus und verschließt sie durch eine Naht, damit sie nicht geschlechtsfähig sind““ ${ }^{38}$ Das Ziel einer solchen Kastration dürfte selten erreicht worden sein. Es ist kaum vorstellbar, dass viele Sauen eine derartig brutale Behandlung, vor allem bei den damaligen Kenntnissen der Sterilität, überlebt haben. Dass eine solche Praxis auch in der Antike nicht gang und gäbe war, lässt sich durch die Aussage Columellas bestätigen, der einen besonderen Beweggrund für das Entfernen der Gebärmutter vermutet: „doch kann ich mir dies nicht anders als aus Futtermangel erklären; denn wo es reichlich Futter gibt, ist es immer vorteilhaft, Nachwuchs aufzuziehen“. ${ }^{39}$

Wirtschaftlich rentabler war da schon die Kastration eines männlichen Schweins. Eber werden und wurden meistens sehr jung kastriert, um etwaige Kämpfe zu vermeiden, und um rasch zu einem fetten, schlachtreifen Schwein zu kommen. Aber auch Kastration in höherem Alter wurde, wie Columella berichtet, durchgeführt: „Die Eber werden entweder halbjährig mit dem Eintritt der Geschlechtsreife oder nach mehrmaliger Fortpflanzung drei- oder vierjährig kastriert, damit sie Fett ansetzen können" ${ }^{40}$ In letzterem Fall wird eine Kastration für das Tier jedoch schmerzhafter. So wurde bei „neuzeitlichen“ Versuchen festgestellt, dass ältere Schweine offensichtlich noch fünf Tage nach dem Eingriff Schmerzen hatten, wohingegen im Alter von zwei bis drei Wochen weniger negative Auswirkungen festgestellt werden konnten. ${ }^{41}$ Einen Vorteil bringt die Kastration - dem typischen Ebergeruch des Fleisches wird dadurch entgegengewirkt. Allerdings wurden zum Beispiel im England der zweiten Hälfte des 20. Jhd. n. Chr. ca. zwei Drittel aller männlichen Mastschweine unkastriert auf den Markt gebracht. ${ }^{42}$ Vor allem bei jung geschlachteten Tieren hält sich der Ebergeruch noch in Grenzen. Die heutzutage nicht mehr gefragte

\footnotetext{
${ }^{34}$ Siehe S. 12.

${ }^{35}$ Hörning 1992, 37.

${ }^{36}$ Plin.nat.8,77,207.

${ }^{37}$ Plin.nat.8,77,209.

${ }^{38}$ Colum.7,9,5f.

${ }^{39}$ Colum.7,9,6.

${ }^{40}$ Colum.7,9,4f.

${ }^{41}$ Hörning 1992, 109.

${ }^{42}$ Hörning 1992, 96.
} 
Verfettung der Schweine (v. a. der Kastraten) ${ }^{43}$ schien zur mykenischen Zeit, wie in der klassischen Antike, durchaus erwünscht gewesen zu sein. Dies zeigt sich auch in den Epen Homers, wo des Öfteren von fetten Schweinen ( $\sigma \tilde{v} \varsigma$ бió $\lambda$ os) die Rede ist. ${ }^{44}$

Als Letztes bleibt noch zu klären, welche Zeitspanne die Mast der Schweine umfasste. Damit verbunden steht zu erwägen, welcher Kategorie von Mastschweinen jene der Linear B-Texte zuzuordnen sind. Zu unterscheiden ist dabei die Mast als solche, welche jüngere Tiere betraf, die Fleisch ansetzen sollten, und die Mästung älterer Schweine zur Gewinnung von reichlich Fettansatz. ${ }^{45}$ Zunächst gibt es zwei Möglichkeiten der Fettmästung: die Tiere wurden entweder über mehrere Jahre hinweg intensiv gemästet, was einen enormen wirtschaftlichen Aufwand bedeutet hätte, oder aber, sie wurden innerhalb kürzester Zeit zur Schlachtung bereitet. Letzteres scheint wohl in Anbetracht des Aufwandes einer intensiven Mast - und um solche wird es sich zumindest bei den Schweinen der Tafel Cn $\mathbf{6 0 8}$ gehandelt haben - am ehesten in Frage zu kommen. Dies wird schon aufgrund der geringen Anzahl verzeichneter Schweine - insgesamt 25 Stück - und des ausdrücklichen Auftrages zur Mästung - jo$a$-se-so-si si- $a_{2}$-ro o-pi-da-mi-jo (Zeile .1.2) - „How the local inhabitants will fatten fat hogs ${ }^{\star 46}$ - bestätigt. Dürfen wir also annehmen, dass es sich hierbei vorrangig um eine Mästung zur Ansetzung von Fett gehandelt hatte und somit ältere Tiere Verwendung fanden? Nach Plinius d. Ä. konnten Schweine in 60 Tagen gemästet werden, „und zwar am besten, wenn die Mast nach dreitägi-

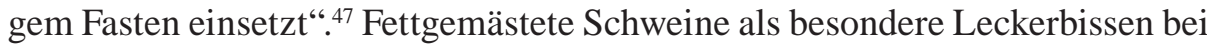
Tisch bevorzugten schon die Helden der Epen Homers. Dies zeigt sich im 14. Gesang der Odyssee, in dem beschrieben wird, dass der Schweinehirt Eumaios für seinen Gast Odysseus ein fünfjähriges Schwein als Gastmahl auftischt: „Her mit dem besten Eber! [...] Die andern brachten indessen den feistesten Eber, er hatte fünf Jahre, ...“. ${ }^{48}$ Die genaue Altersangabe scheint dichterische Konvention gewesen zu sein, da sowohl in der Ilias als auch in der Odyssee diese Altersangabe mehrmals (für Schweine und Rinder) erfolgte. ${ }^{49}$ Trotzdem zeigen diese Stellen die Vorliebe für ältere Tiere, die hauptsächlich zum Fettansatz geeignet waren.

Aus den angeführten Argumenten, die für eine kurze intensive Mast zum Ansatz von Fett sprechen, möchte ich die Tafel Cn 608 als Auftrag zur Vorbereitung für eine oder mehrere Festlichkeit/en sehen. Diese sind wohl in Zusammenhang mit Banketten zu verstehen, im Zuge derer die Schweine geschlachtet und gegessen wurden. Mastschweine sind, wie schon gesagt, auch in der $\mathbf{U}$ Serie von Pylos (PY Ua 25.1, Un 2.6, Un 138.4) verzeichnet, wobei durch den Inhalt von Un 2 eindeutig ein Bezug zu einer Feierlichkeit festzustellen ist. ${ }^{50}$ Wenn die Mastschweine dieses Textes für eine größere Feier zur Verfügung standen, scheint es nicht abwegig, dass solche Tiere schon früher (im Falle von PY Cn 608.2 bei den Gemeindeeinwohnern, den o-pi-da-mi-jo) bestellt wurden. Des Weiteren ist zu bedenken, dass die Tafel $\mathbf{C n} 608$ von derselben

\footnotetext{
${ }^{43}$ Hörning 1992, 109.

${ }^{44}$ Zum Beispiel in Hom.Od.2,300; 14,81; 17,80; 20,251 und Hom.Il.9,208; 21,263.

45 Siehe dazu S. 10 u. Fn. 33.

${ }^{46}$ Ventris-Chadwick 1973, $205 f$.

${ }^{47}$ Plin.nat.8,77,208.

${ }^{48}$ Hom.Od.14,414ff.

${ }^{49}$ Hom.Il.2,403 (Stier); Hom.Il.6,315 (Stier); Hom.Od.14,419 (Schwein); 19,420 (Schwein); 10,390 wird ein neunjähriges Schwein angeführt.

${ }^{50}$ Siehe S. 8f.
} 
Schreiberhand (S 608 - H 1) beschrieben wurde wie PY Cn 3. Nachdem letzterer Text aufgrund seines Inhaltes eindeutig in den kultischen Bereich fällt, ${ }^{51}$ ist dies nach den vorhandenen Tafelbelegen auch für Cn 608 wahrscheinlich. Damit könnten auch die Mastschweine der Tafeln PY Ua 25, Un 138 sowie der Tonplomben TH Wu 52. $\alpha$ und Wu 68. $\alpha$ als Tiere zu sehen sein, die für eine Feier mit Bankettmahl gedacht waren.

\subsection{DAS IDEOGRAMm SUS $+K A$}

PY

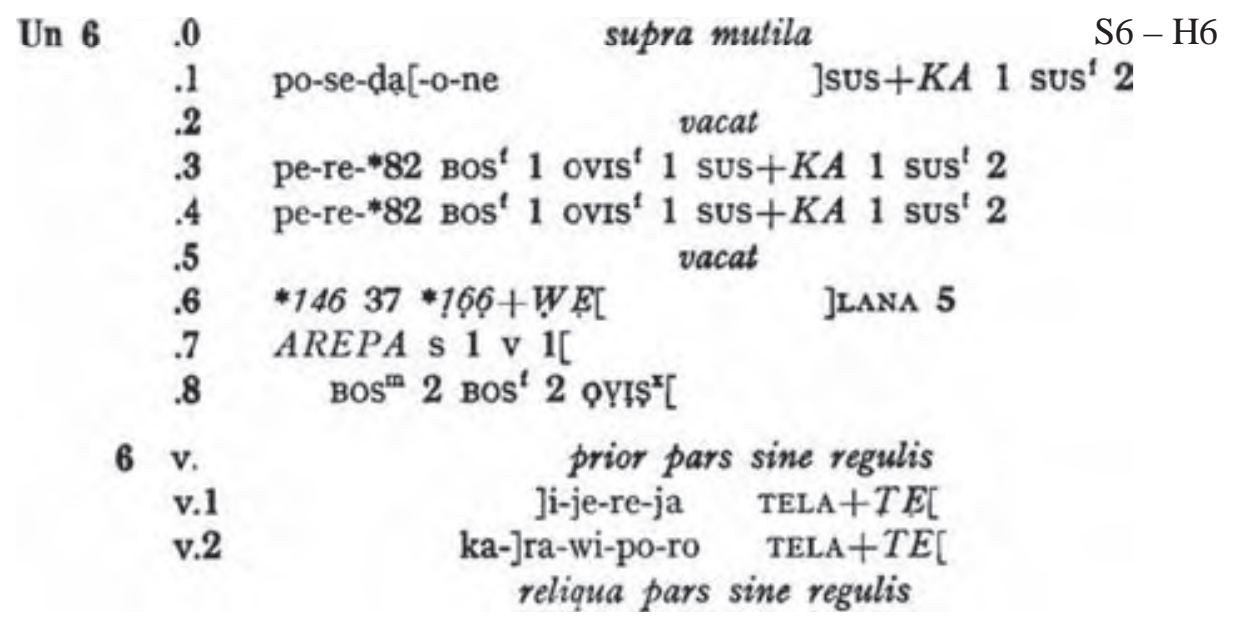

PY
Un $853[+] 869$ (pars dextra superior) [+] 870 (dextra inferior) [+] 876 (sinistra inferior]

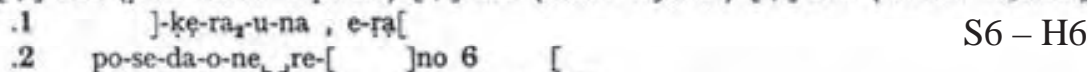

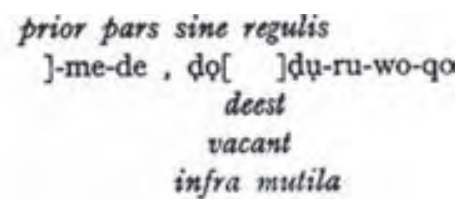

Eine weitere Kategorie von Schweinen - definiert als sus $+K A$ - findet sich nur in zwei Texten aus Pylos, und zwar in PY Un 6 und Un 853. Auffällig ist, dass zu dem Ideogramm sus $+K A$ bei allen belegten Stellen die doppelte Anzahl an weiblichen Schweinen (sus ${ }^{\mathrm{f}}$ ) hinzugefügt ist. Auf PY Un 6.1.3.4 steht jeweils sus $+K A 1$, sus ${ }^{f} 2$ und auf PY Un 853.5 sus $+K A 2$ 2, sus 4 geschrieben. Die Schweine waren dabei auf beiden Texten für den Gott Poseidon gedacht, und nach PY Un 6 für eine weibliche Gottheit namens pe-re-*82, für die zusätzlich zweimal eine Kuh und ein weibliches Schaf (Zeile .3.4) verzeichnet sind. ${ }^{52}$ Diese Göttin stand offensichtlich in Verbindung zu dem Poseidonheiligtum von Pylos,

51 Zur Interpretation der Tafel PY Cn 3 s. S. 30ff.; vgl. auch R. Palmer 1994, 77.

52 Bartoněk 2003, 421; Chadwick 1976, 95. 
da sie auch auf PY Tn 316 in Zusammenhang mit ebendiesem genannt wird. ${ }^{53}$ In Zeile .1 von Un 6 könnten für Poseidon selbst die gleichen Tiere oder auch männliche Gegenstücke verzeichnet gewesen sein; E. L. BENNETT nimmt BOS und ovis ${ }^{f}$ als zu ergänzen an, während N. YAMAGATA diese oder BOS ${ }^{m}$ und ovIS ${ }^{\mathrm{m}}$ in Betracht zieht. ${ }^{54}$ Zeile .8 nennt dann noch zwei männliche und zwei weibliche Rinder und Schafe unbestimmten Geschlechts und Anzahl. Hingegen verzeichnen die Zeilen .6 und .7 weitere Gaben: Gewänder $(* 146 \text {, *166+WE) })^{55}$, Wolle (LANA) und Salbe (AREPA) ${ }^{56}$. Die Angaben der Priesterin (]i-je-re-ja) und der Schlüsselträgerin ( $k a-$ ra-wi-po-ro) in Verbindung mit Textilien (TELA + TE[) müssen ungeklärt bleiben ${ }^{57}$ Auch ein Stifter wird nicht genannt und aufgrund dessen scheint es wahrscheinlich, dass es sich um Gaben handelt, die vom Palast selbst gestellt wurden. ${ }^{58}$ Da dies keiner näheren Ausführung bedurfte beziehungsweise bei einer fehlenden Angabe der Palastadministration klar war, wer gemeint war, entfiel offensichtlich die Angabe des Gabenstellers.

Anders verhält es sich auf der Tafel PY Un 853. Hier wird ]-ke-ra $a_{2}-u$-na als Überbringer der Gaben erwähnt. Der Name wird mit $e-k e-r a_{2}$-wo von PY Un 718.2 in Verbindung gebracht, wobei es sich hier wiederum um einen Gabenbringer handelt. Damit wäre ]-ke-ra $a_{2}-u-n a / e-k e-\mathrm{ra}_{2}-w o$ als eine Person gehobener Stellung zu sehen, ${ }^{59}$ die verschiedene Abgaben in kultischem Zusammenhang tätigte. Die gelieferten Tiere und sonstigen Produkte sind ähnlich jenen der Tafel PY Un 6, nur gelten sie diesmal für den Gott Poseidon alleine. An Tieren sind auf Un 853 abermals männliche und weibliche Schweine (Zeile .5), ein weibliches Schaf und anstelle der Rinder ein männliches Schaf und eine Ziege (Zeile .4) verzeichnet.

In Bezug auf die Schweine der beiden Tafeln scheint das determinative Silbenzeichen $K A$ zur Bezeichnung eines Ebers beigefügt worden zu sein und wurde von M. Ventris und J. CHADWICK mit dem klassisch-griechischen Wort

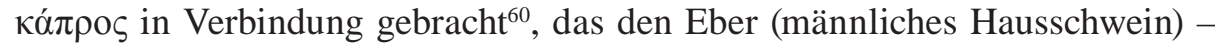
aber auch den Keiler (männliches Wildschwein) - bezeichnet. Für das Wildschwein gab es im klassischen Griechisch jedoch noch einen zweiten Aus-

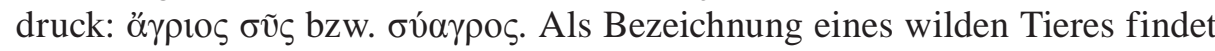
sich in den Linear B-Texten der Begriff a-ki-ri-ja auf KN C(2) 7064.1 den Ideogrammen von männlichen und weiblichen Ziegen zugefügt. Das Wort $a$ ki-ri-ja wurde bereits in Documents in Mycenaean Greek mit dem klassischen örpios in Verbindung gebracht. ${ }^{61}$

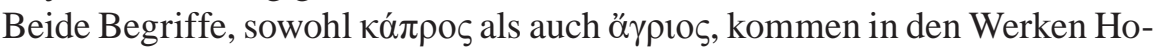
mers, vor allem in der Ilias, als Bezeichnung eines wilden Schweins vor. Dabei ist jedoch nicht in allen Fällen eindeutig ein Keiler gemeint, vielmehr wird ein

53 Auf PY Tn 316v.4.5 ist pe-re-*82 als Empfängerin von Gaben und Personen (AUR *213 ${ }^{\mathrm{VAS}}$ und MUL 1) angeführt. Zeile v.1 nennt das Heiligtum des Poseidon ( $p o-s i-d a-i-j o$ ) als Ort, wohin die Gefäße und Frauen für die unterschiedlichen angeführten Gottheiten gebracht werden.

${ }^{54}$ Bennett 1992, 105; Yamagata 1995, 60.

55 Duhoux 1974, 128.

${ }^{56}$ Gewänder, Wolle und Salböl.

57 Vgl. Weilhartner 2002, 164.

${ }^{58}$ R. Palmer 1994, 101

59 Zur Identifikation des ]-ke-ra $a_{2}$-u-na/e-ke-ra $a_{2}$-wo s. Lindgren 1973 II, 153f.; de Fidio 1977, 131ff.; Lejeune 1973, 64; L. R. Palmer 1963, 216; Chadwick 1987, 78 Fn. 6; Palaima 1995, 129; Leukart 1992, 391ff.; Killen 1999, 352f.

${ }^{60}$ Ventris-Chadwick 1973, 549.

${ }^{61}$ Ventris-Chadwick 1973, 529; Killen-Olivier 1968, 136. 
Vergleich zu der Wildheit eines männlichen Schweins angestellt. ${ }^{62}$ Ein schönes Beispiel aus der Odyssee zeigt, dass die Männlichkeit (sprich das Nicht-kastriert-

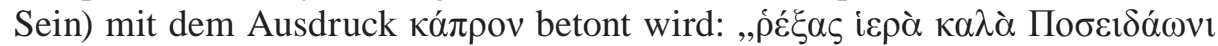

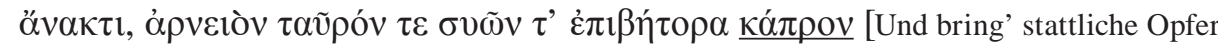
dar dem Herrscher Poseidon, einen Widder und Stier und einen springenden Eber] “ ${ }^{63}$ Könnte dies nicht ein Hinweis darauf sein, dass in mykenischer Zeit dementsprechend das Ideogramm sus $+K A$ ein nicht kastriertes Schwein bezeichnete? ${ }^{64}$

\subsection{KASTRIERTE UND UNKASTRIERTE MÄNNLICHE SCHWEINE}

Auffällig an den Texten der pylischen $\mathbf{U}$-Serien ist das Fehlen des Ideogramms für männliche Schweine sus ${ }^{\mathrm{m}}$. Verzeichnet sind $\operatorname{sus}^{\mathrm{f}}$, sUS $+S I$, sUS $+K A$ und sus. Wie bereits weiter oben erwähnt, ist auffällig, dass neben sus $+K A$ immer die doppelte Anzahl an weiblichen Schweinen verzeichnet ist. Dagegen folgen auf PY Un 2.6 auf ein Mastschwein ebenfalls weibliche Schweine - hier sechs Stück. Ob die Anzahl der weiblichen Tiere eine Bedeutung hat, möchte ich außer Acht lassen, da es aussichtslos scheint, darüber zu diskutieren. Die Schlussfolgerung nach den vorhergehenden Kapiteln lautet jedenfalls, dass wenn das Ideogramm sus ${ }^{f}$ für weibliche Schweine verzeichnet ist, nicht aber sus $^{\mathrm{m}}$, die männlichen Tiere unter den Zeichen sus $+S I$ und sus $+K A$ zu suchen sind. Nach den Ausführungen in dem Kapitel über Mastschweine ${ }^{65}$ sind unter sus + SI fettgemästete und kastrierte männliche Schweine zu verstehen, während das Ideogramm sus $+K A$ wohl für unkastrierte männliche Schweine Verwendung fand. Dass letztere nicht nur mit sus ${ }^{\mathrm{m}}$ definiert, sondern mit einem speziellen Ausdruck versehen wurden, scheint nicht abwegig, wenn man bedenkt, dass auch in klassischer Zeit für den Kult ausdrücklich unkastrierte Tiere verlangt wurden. ${ }^{66}$ Die Frage, die jedoch noch zu berücksichtigen ist, lautet: wurden männliche Schweine, bis auf einige Ausnahmen für die Zucht und den Kult, kastriert? Oder, waren nur die Mastschweine dieser Prozedur unterworfen worden? Zumindest in römischer Zeit ist die Kastration üblich; so berichtet Columella, wie bereits weiter oben angeführt, dass Schweine entweder halbjährig oder drei- bis vierjährig kastriert wurden. ${ }^{67}$ Damit könnten mit dem Ideogramm sus ${ }^{\mathrm{m}}$, wie in den Texten von Pylos und Knossos vorkommend, generell kastrierte männliche Schweine gemeint gewesen sein. Auch ist nach den vorhergehenden Ausführungen ${ }^{68}$ kaum anzunehmen, dass alle verschnittenen männlichen Schweine als Fett-Mastschweine (sus + SI) gehalten wurden. In der pylischen $\mathbf{C n}$-Serie sind männliche Schweine mit dem Ideogramm sus ${ }^{\mathrm{m}}$ definiert (PY Cn 45 lat.inf. sus ${ }^{\mathrm{m}}$ 11, Cn $\mathbf{6 0 0 . 1 4}$ sus $^{\mathrm{m}}$ 30, Cn 925.1 sus $^{\mathrm{m}}$ 16); das gleiche Zeichen ist in der knossischen Co-Serie (KN Co 907.2 sus $^{\mathrm{m}}$ 21, Co $\mathbf{9 0 9 . 2}$ sus ${ }^{\mathrm{m}}$ 3) zu finden. ${ }^{69}$ Auf KN Co $\mathbf{9 0 3 . 2}$ gab der

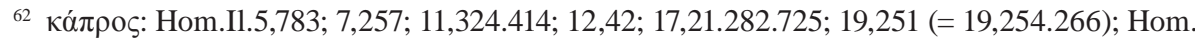

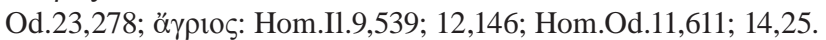

${ }^{63}$ Hom.Od.11,130f.; mit ähnlichem Wortlaut nochmals in Hom.Od.23,277f.

${ }_{64}$ Dieselbe Meinung vertritt John T. Killen in der Neuauflage der Documents in Mycenaean Greece (Auskunft per Brief vom 1. August 2005).

65 Siehe S. 7ff.

${ }^{66}$ Ziehen 1939, 593.

${ }^{67}$ Colum.7,9,4f.

${ }^{68}$ Siehe S. 11ff.

${ }^{69}$ Godart 1971b, 92 verstand die Ideogramme ovIS ${ }^{\mathrm{m}}$, CAP ${ }^{\mathrm{m}}$, sus ${ }^{\mathrm{m}}$ und BOS $^{\mathrm{m}}$ der knossischen Co-Serie als Definitionen von männlichem unkastrierten Zuchtvieh. Doch kann dies aufgrund meiner Interpretation (s. S. 16f., 19ff.) dieser Ideogramme als Zeichen für kastrierte männliche Tiere nicht angenommen werden. 
Schreiber H 107, der für die Abfassung aller Tafeln der Co-Serie zuständig war, männliche Schweine mit dem Ideogramm sus an. Hierbei könnte von einem unabsichtlichen Weglassen der männliche Tiere kennzeichnenden horizontalen Striche ausgegangen werden, da der Schreiber bei den sonstigen Ideogrammen die Geschlechter immer in weibliche Tiere und männliche kastrierte Tiere unterschied.

Auf den thebanischen Tonplomben sind alle Ideogramme der verschiedenen Gattungen von Schweinen, außer sus $+K A$ vertreten. Dass dieses nicht vorkommt, scheint wohl dadurch begründet, dass nur ein Teil der tatsächlich einmal vorhandenen Tonplomben gefunden wurde, sodass man schon aufgrund dessen nicht erwarten kann, dass alle Tierideogramme auftreten, oder aber dass zum Zeitpunkt der Ankunft der anderen Tiere einfach keine unkastrierten männlichen Schweine geliefert wurden..$^{70}$ Das einfache Ideogramm sus ist auf TH Wu 58. $\alpha$ zu finden, sus ${ }^{\mathrm{m}}$ auf TH Wu 47. $\alpha$, Wu 51. $\alpha$, Wu 55. $\alpha$, Wu 60. $\alpha$, Wu 66. $\alpha$ und Wu 75. $\alpha$. Die weiblichen Tiere - sus $^{\mathrm{f}}-$ sind auf TH Wu 63. $\alpha$ und Wu 96. $\alpha$ verzeichnet und ein Tier, dessen Geschlecht nicht mehr zu

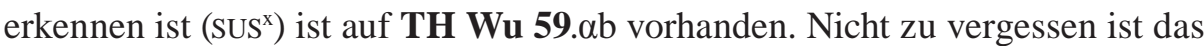
bereits besprochene Ideogramm sus + SI der Tonplomben $\mathbf{W u ~ 5 2 . \alpha}$ und $\mathbf{W u}$ 68. $\alpha$.

\subsection{KASTRIERTE UND UNKASTRIERTE MÄNNLICHE RINDER}

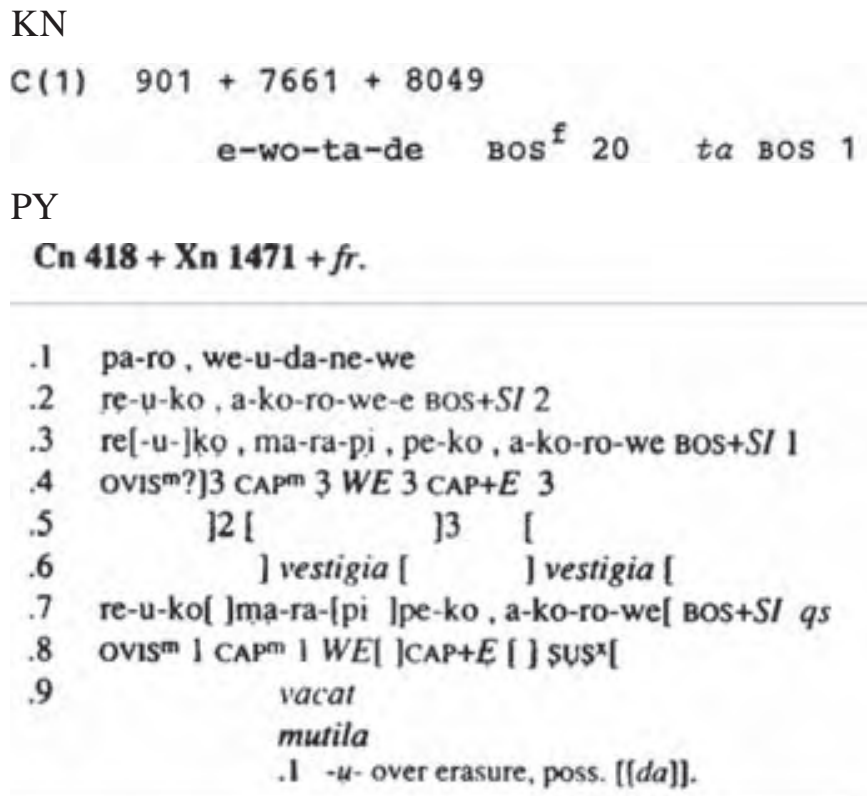

.1 pa-ro, we-u-da-ne-we

.2 re- $u$-ko, a-ko-ro-we-e Bos $+S I 2$

.3 re[-u-]ko, ma-ra-pi, pe-ko, a-ko-ro-we BoS+SI 1

.4 ovis $^{m}$ ?] 3 CAPm 3 WE 3 CAP $+E 3$

.7 re-u-ko[ ]ma-ra-[pi ]pe-ko, a-ko-ro-we[ Bos+SI qs

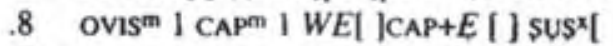

9

Analog zu den zuvor behandelten Ideogrammen sus + SI und sus $+K A$ scheinen mir auch die in den Linear B-Texten vorkommenden Rinderideogramme eingeteilt werden zu können. Hierbei ist vor allem die Tafel PY Cn 418 zu nennen. In Zeile .1 ist der Namen we-u-da-ne-we (Dativ von we-u-da-ne-u), welcher mit der Präposition pa-ro („bei“ oder „von“) verbunden ist, verzeichnet. In Cn 418.2-.5.7.8 folgen die unterschiedlichsten Tierideogramme: BOS +SI, ovIS ${ }^{\mathrm{m}}[$ ?],

\footnotetext{
${ }^{70}$ So ist in der Wu-Serie von Theben auch das Ideogramm Bos + SI (Mastrind) nicht zu finden.

${ }^{71}$ Join nach Melena 1994-95, $95 f$.
} 
$\mathrm{CAP}^{\mathrm{m}}, W E$, BOS ${ }^{\mathrm{x}}\left[\right.$ ?], CAP $+E$ und sUS ${ }^{\mathrm{x}} .^{72}$ Die auf $\mathbf{C n} 4 \mathbf{4 1 8}$ aufgelisteten Tiere umfassen also männliche kastrierte Schafe und Ziegen, Jungtiere, ${ }^{73}$ Schweine, Rinder und die noch zu besprechenden Ziegen ${ }^{74}$ welche mit dem Ideogramm $\mathrm{CAP}+E$ definiert wurden. Bei den als BOS $+S I$ bezeichneten Rindern der Tafel PY Cn 418.2.3 handelte es sich nicht um „einfache“ Rinder, sondern, nach dem Ideogramm Bos und der Ligatur SI zu schließen, um Mastrinder, was deren Gebrauch für alltägliche Zwecke wohl ausschließt. ${ }^{75}$ Dies bedeutet, dass es männliche Tiere gewesen sein mussten, die höchstwahrscheinlich kastriert waren. Gleich wie Schweine sind auch Ochsen besser zur Mast geeignet und setzen das in der Antike und bei Homer so begehrte Fett an. ${ }^{76}$ Die in dem Text verzeichneten Mastrinder sind auf ihre körperlichen Eigenschaften - re-u-ko (, ma-ra-pi , pe-ko ,) a-ko-ro-we(-e) - hin, ausführlicher beschrieben. TH. PALAIMA hat die unterschiedlichen Auslegungen der Wörter in seinem Artikel The Pylos Oxen Tablets zusammengefasst. ${ }^{77}$ Übereinstimmung gibt es bei der

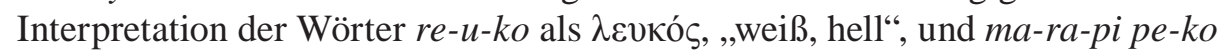

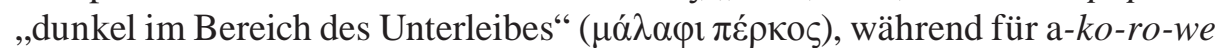
verschiedene Varianten („nicht kastriert“ (akolōw(w)ēs?), „ohne Makel/Farbe“ (a-khrōwēs), „,von einheitlicher Farbe“ ((h)a-khrōwēs)) vorgeschlagen wurden. ${ }^{78}$ TH. PALAima sprach sich bedingt für die Interpretation L. R. PALMERS als zu

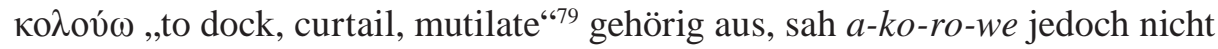
wie L. R. PALMER im Zusammenhang mit „,uncastrated““ ${ }^{80}$ sondern ausgehend

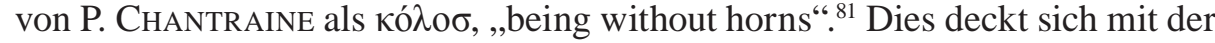
Deutung des Ideogrammes BOS +SI als Zeichen für kastrierte Tiere. Vielleicht sind auf Cn 418 Ochsen verzeichnet, die ursprünglich als Arbeitstiere vorgesehen waren oder dienten, dann jedoch für die Fettmästung herangezogen wurden. ${ }^{82}$ Nach TH. Palaima war es zumindest durchaus üblich, Arbeitsochsen die Hörner abzuschneiden. ${ }^{83}$

Das Anführen der körperlichen Eigenschaften der Tiere kann auf den kultischen Bereich der mykenischen Kultur hinweisen. Zum einen wurden Rinder, wie Schweine, wohl für spezielle Anlässe fett gemästet, ${ }^{84}$ und zum anderen zeigt sich durch einen Vergleich mit den Kultpraktiken des klassischen Griechenlands, dass die Farbe und Beschaffenheit von Tieren bei den Opferriten von Bedeutung war. ${ }^{85}$ Nachdem die als sus + SI bezeichneten Schweine für größere Festlichkeiten gedacht waren ${ }^{86}$, dürfen wir annehmen, dass fett ge-

72 Join nach Melena 1996-97, 176.

${ }^{73}$ Diese in Zeile .4 und .8 als WE (*wetalon) verzeichneten Tiere sind wohl als junge Ziegen zu sehen, da $W E$ jeweils auf das Ideogramm $\mathrm{CAP}^{\mathrm{m}}$ folgt.

${ }^{74}$ Siehe S. 38f.

75 Killen 1996, 77.

${ }^{76}$ Benecke 1994, 177; vgl. Kraemer 1940, $1176 f$.

77 Th. Palaima: Perspectives on the Pylos Oxen Tablets: Textual (and Archaeological) Evidence for the Use and Management of Oxen in Late Bronze Age Messenia (and Crete); das ausführliche Zitat s. in der angefügten Literaturliste unter Palaima 1989.

${ }^{78}$ Palaima 1989, 104ff.; s. auch Aura Jorro 1985 s.v. a-ko-ro-e-we, s.v. ma-ra-pi; Aura Jorro 1993 s.v. re-u-ko; L. R. Palmer 1963, 432; Bartoněk 2003, 265.

79 L. R. Palmer 1966, 283; L. R. Palmer 1981, 1502.

${ }^{80}$ Palaima 1989, 106.

${ }^{81}$ Palaima 1989, 106; Chantraine 1984, s.v. kódos.

${ }^{82}$ Palaima 1989, 107.

${ }^{83}$ Palaima 1989, 107: „,... that it is fairly standard practice to cut off the horns of worker oxen.“

${ }^{84}$ Siehe S. 12.

${ }^{85}$ Ziehen 1939, 592ff.

${ }^{86}$ Siehe S. 12f. 
mästete Rinder (BOS + SI) einer ähnlichen Bestimmung zugeführt wurden. Das Ideogramm BOS + SI drückte wahrscheinlich nicht speziell aus, dass dieses Tier verschnitten war, sondern vor allem, dass es fett gemästet wurde, was natürlich bei einem Ochsen leichter zu bewerkstelligen ist.

Der einzige Nachweis für ein als Stier betontes Rind findet sich in Knossos auf der Tafel KN C(1) 901, wo dem Ideogramm Bos das Silbenzeichen ta ( $\tau \alpha \tilde{v} \rho \varsigma_{\text {) }}$ ) vorgestellt ist. Dieses Ideogramm darf wohl analog zu sus $+K A$ der Tafeln PY Un 6.1.3.4 und Un 853.5, welches nicht verschnittene Tiere darstellt, sowie entsprechend der klassischen Verwendung des Wortes $\tau \alpha \tilde{v} \rho \circ \varsigma$ „Stier, Bulle“ als Bezeichnung eines nicht kastrierten männlichen Rind gesehen werden. Der Text nennt des Weiteren den Ort $e$-wo-ta(-de $)^{87}$ und 20 Kühe (Bos $\left.{ }^{\mathrm{f}}\right)$. J. T. KILLEN stellte 1996 in seinem Artikel Thebes Sealings and Knossos Tablets die Tafel C(1) 901 in den Bereich des Kultes, wobei er einräumte, dass $e$-wota-de „to the festival“ ( $\dot{\varepsilon} \circ \tau^{\alpha} \alpha \dot{v \delta \varepsilon}$ ) heißen könnte. ${ }^{88}$

Vielleicht handelte es sich jedoch auch um Tiere, die für die Zucht gedacht waren. Vor allem die Erwähnung eines Stieres in Zusammenhang mit Kühen könnte darauf hindeuten. Die Rinder wären demnach zur Zucht nach e-wo-ta gesandt worden oder dort zu lokalisieren. ${ }^{89}$ Diese Deutung wird durch Angaben antiker Autoren unterstützt. So gibt der römische Agrarschriftsteller Columella an, dass ein Bulle für 15 Kühe genügt, während Marcus Terentius Varro für 70 Kühe zwei Stiere rechnete. ${ }^{90}$ Bereits 1966 schlugen J. T. KILlen und J.-P. OLIVIER vor, in den auf der Tafel KN C(1) 901 verzeichneten 20 Kühen und dem einen Stier eine Herde zu sehen: „... mais il semble préférable d'y voir une abréviation de tauros [...] devant l'idéogramme du bovin indifférencié [...]; la proportion d'un taureau pour 20 vaches inciterait à voir ici la mention d'un troupeau rèel .....911

\subsection{Kastrierte Und UnKastrierte TIERE IN DEN LineAr B-TeXteN}

\section{$\mathrm{KN}$}

Ce 50 Zatus superius

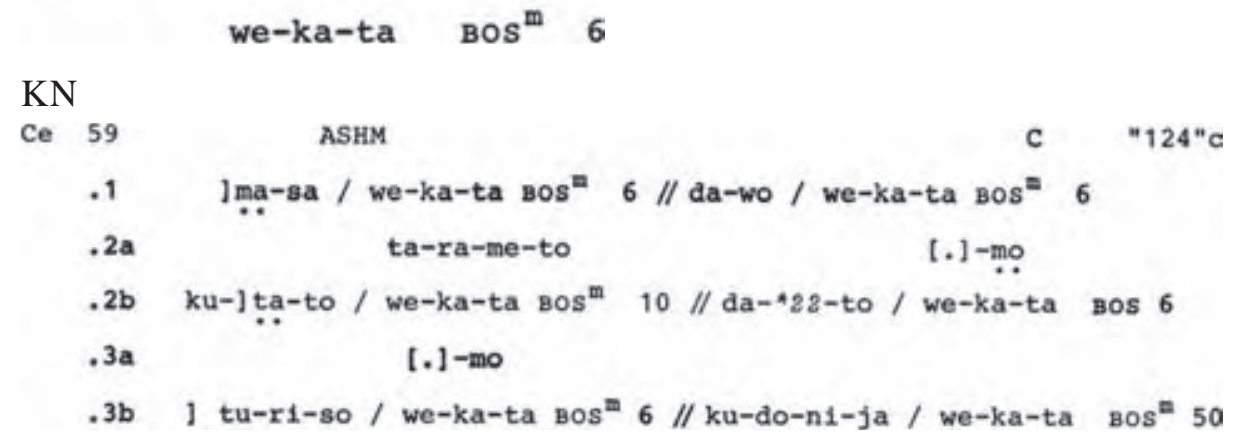

\footnotetext{
${ }^{87}$ Aura Jorro 1985, s.v. e-wo-ta-de; Ventris-Chadwick 1973, 547.

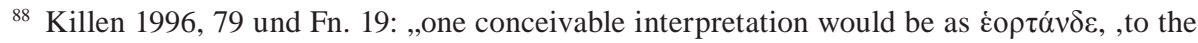
festival“ “ (Bezug nehmend auf: „C. Milani, Aevum 44 (1970) 306“).

${ }^{89}$ Ich danke Frau Prof. Dr. Sigrid Jalkotzy für diesen Vorschlag.

${ }^{90}$ Colum.6,24,3; Varro rust.2,5,12.

${ }^{91}$ Killen-Olivier 1966, 61.
} 
PY

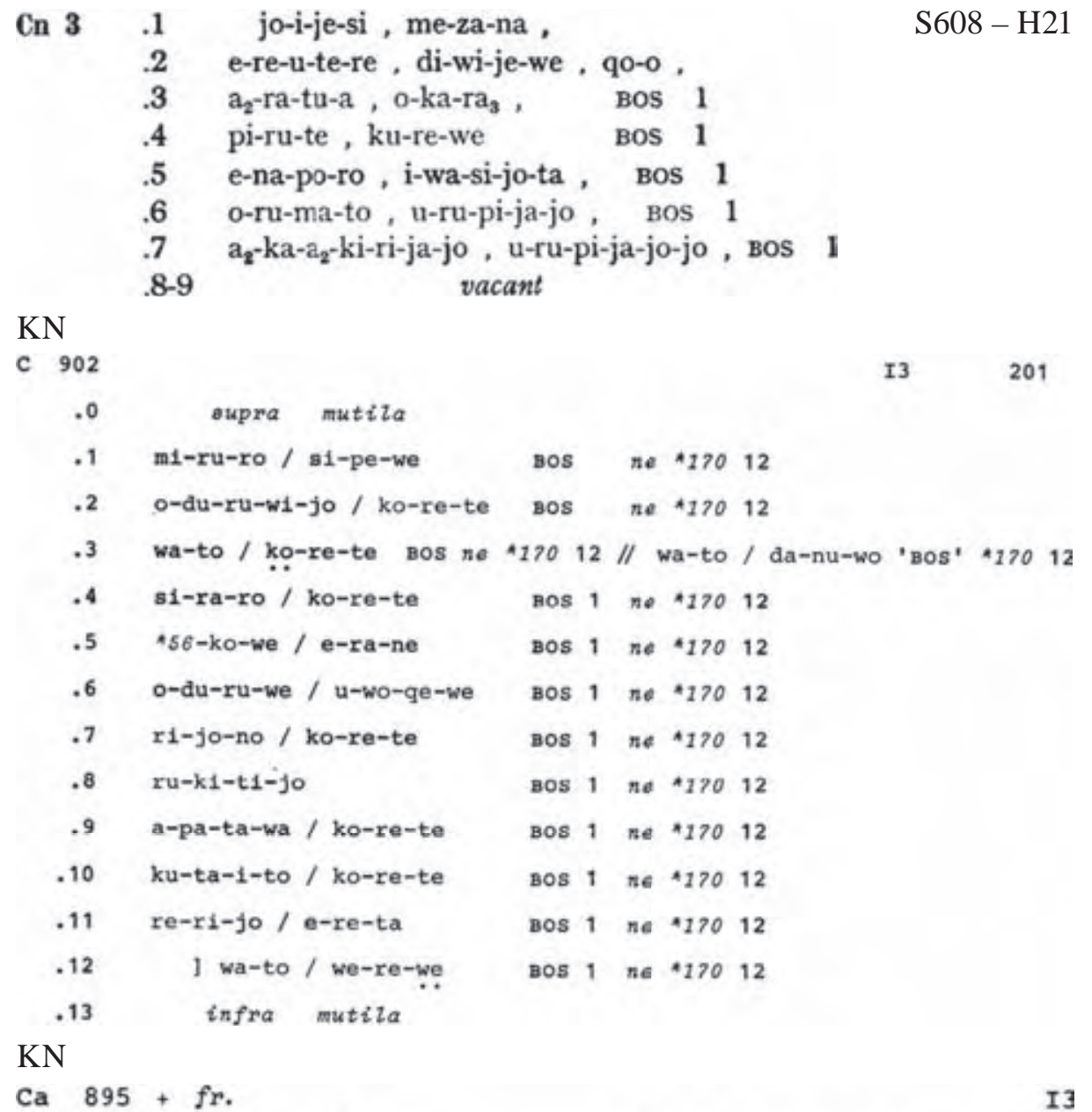

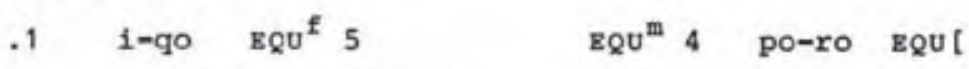

.2 o-no $\varepsilon_{Q U^{f}} 3$ po-ro $\mathrm{EQU}_{2} 2 \mathrm{EQU}^{\mathrm{m}} 4$ [

PY

Cr 591.0

\begin{tabular}{lll}
.0 & \multicolumn{3}{c}{ supra mutila } \\
cerv 3 \\
.1 & ]-wa & $\begin{array}{c}\text { CERV } 3 \\
.2\end{array}$ \\
.3 & ]ne-wi & CERV 2[ ] $v$. \\
.4 & ]wa & CERV 1[ ] $v . ~$ \\
.5 & ]na-[ ] CERV [ ]2 \\
.6 & & CERV ]2 \\
.7 & & CERV] 3
\end{tabular}

PY

Cr $868+\operatorname{Cr} 875$
$.1 \mathrm{a}_{3}$-se-we
[CERV qs
.2 ne-se-e-we
CERYL $q s$
.3 te-re-ne-wi-ja
CERV[ $q s$
.4 na-pe-re-wa
CERVI $q s$
.5 Jto-na-pe []
CERYI qs
6 Ina-wo
CERV 1
.7 qe-re-me-ti-wo[ ] CERV I
.8-10
vacant
infra mutila

(Cr; S868-Ci] 
Wie aus den beiden vorhergehenden Kapiteln über kastrierte und unkastrierte männliche Schweine sowie kastrierte und unkastrierte männliche Rinder ersichtlich, könnten die in den pylischen und thebanischen Linear B-Texten aufgelisteten Ideogramme sus + SI (PY Cn 608.3-.11, Ua 25.1, Un 2.6, Un 138.4; TH Wu 52. $\alpha$, Wu 68. $\alpha$ ) und BOS + SI (PY Cn 418.2.3) als Zeichen für maskuline verschnittene Tiere (Mastschweine und Mastrinder) zu verstehen sein, während unter dem Zeichen sus + KA der Tafeln PY Un 6.1.3.4 und Un 853.5 unverschnittene Schweine (Eber) zu vermuten wären. Genauso ließe sich die Angabe $t a$ BOs aus dem Text KN C(1) $901+7661+8049$ als Ideogramm eines ebensolchen Rindes (Stier) annehmen. ${ }^{92}$ Neben diesen genannten Tieren sind in den Linear B-Texten die Ideogramme für die jeweiligen weiblichen Pendants verzeichnet - sus ${ }^{\mathrm{f}}$ für Sauen ${ }^{93}$ und Bos $^{\mathrm{f}}$ für Kühe ${ }^{94}$. Dazu kommen noch die Zeichen SUS $^{\mathrm{m}}$, BOS ${ }^{\mathrm{m}}$, sus und Bos, wobei die beiden ersteren als männliche Tiere $\mathrm{zu}$ verstehen sind. Nicht einzuordnen war bislang das Geschlecht der mit den Ideogrammen sus und Bos gekennzeichneten Schweine und Rinder. Auf deren Interpretation soll im Folgenden ausführlich eingegangen werden. ${ }^{95}$

Besonders auffällig ist, dass in der gesamten U-Serie von Pylos nie sus ${ }^{m}$ verzeichnet wurde. Dieses Ideogramm findet sich in den pylischen Texten, soweit erhalten, auf PY Cn 45 lat.inf., Cn 600.14 und Cn 925.1, in Knossos vor allem auf KN Co 907.2, Co 909.296 und in Theben auf den Tonplomben TH Wu 47. $\alpha$, Wu 51. $\alpha$, Wu 55. $\alpha$, Wu 60. $\alpha$, Wu 66. $\alpha$.

Im Gegensatz zu sus ${ }^{\mathrm{m}}$ ist in den $\mathbf{U}$-Serien von Pylos Bos $^{\mathrm{m}}$ enthalten - PY Ua 25.1, Un 138.4, Un 718.3; sie sind dafür nicht in der pylischen Cn-Serie zu finden. In Knossos ist Bos $^{\mathrm{m}}$ vor allem in der Co-Serie vertreten - KN Co 903.2, Co 904.2, Co 907.2, Co 7056.2 und auf den Texten KN Ce 59.1.2b.3 und Ce 50 lat. sup., wobei die entsprechenden Rinder auf den letzten beiden Tafeln als we-ka-ta näher definiert sind. Dieses Wort wird mit dem klassisch-griechi-

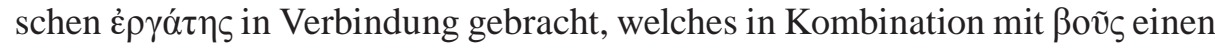
Arbeitsochsen definiert. ${ }^{97}$ Durch die jeweils gerade Anzahl der verzeichneten Tiere und dem Begriff we-ka-ta wird es sich bei den als Bos $^{\mathrm{m}}$ bezeichneten Tieren aus den knossischen Texten um vor den Pflug gespannte Ochsen gehandelt haben.$^{98}$ Verschnittene männliche Rinder sind ruhiger und folgsamer als ihre unverschnittenen Artgenossen und deshalb besser als Pflug- und Lasttiere geeignet. ${ }^{99}$ Die gleiche Interpretation trifft auf das Ideogramm BOS $^{\mathrm{m}}$ der knossischen Ch-Serie zu, welches mit dem zusätzlichen Zeichen $Z E$ versehen ist.

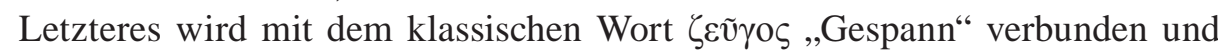
zeigt somit jeweils zwei Stück von Rindern an; dabei wird es sich ebenfalls

${ }^{92}$ Siehe S. 15ff., $16 f$.

${ }_{93}$ Das Ideogramm sus ${ }^{\mathrm{f}}$ ist auf folgenden Tafeln zu finden: PY Cn 440.7, Cn 599.7.8, Cn 600.15, Cn 643.2, Cn 925.2.3, Cn 1116.1, Ua 17.2, Un 2.6, Un 6.1.3.4, Un 853 [+] 869.5; TH Wu 63. $\alpha$, Wu 96. $\alpha$; KN Co 903.2, Co 904.2, Co 906.2, Co 907.2, Co 909.2, Co 910.2, Co 7056.2; hierbei handelt es sich bei den Texten aus Knossos um eine Auswahl der sus ${ }^{\mathrm{f}}$ enthaltenden Tafeln.

${ }^{94}$ Das Ideogramm Bos $^{f}$ findet sich in den Texten: PY Ua 25.1, Un 6.3.4.8, Un 138.4; TH Wu 53. $\alpha$, Wu 76. $\alpha$; KN C(1) 901, Co 903.2, Co 906.2, Co 907.2, Co 7056.2; auch hier gilt wiederum, dass es sich nur um eine Auswahl der in Knossos vorkommenden Texten mit dem Zeichen $\operatorname{BOS}^{\mathrm{f}}$ handelt.

${ }^{95}$ Siehe S. $23 f f$.

${ }_{96} \mathrm{Zu}$ dem Ideogramm sus der Tafel KN Co 903.2 s. im Folgenden S. 27ff.

97 Ventris-Chadwick 1973, 213, 590; Aura Jorro 1993, s.v. we-ka-ta.

98 Ventris-Chadwick 1973, 213.

${ }^{99}$ Über die Gründe und Auswirkungen der Kastration männlicher Tiere s. Benecke 1994, $175 f f$. 
um Arbeitsochsen gehandelt haben, da diese paarweise vor den Pflug gespannt wurden. ${ }^{100}$ Bis auf einige Ausnahmen sind alle männlichen Rinder der genannten Serien mit dem Ideogramm Bos ${ }^{\mathrm{m}}$ wiedergegeben. Die Ausnahmen betreffen Tiere, die mit dem bloßen Zeichen *109 вos (Ce 59.2b, Ce 144.1, Ce 7061.1.2, Ch 7047, Ch 7066, Ch 7100 + 7703) verzeichnet sind. Hierbei könnte es sich um Verschreibungen der jeweiligen Schreiberhände handeln, wobei die horizontalen Striche einfach vergessen wurden. ${ }^{101}$ Es muss jedoch die Möglichkeit offen gelassen werden, dass es sich keinesfalls um eine Verschreibung handelt, sondern in diesen Fällen sehr bewusst auf die zwei horizontalen Striche verzichtet wurde. ${ }^{102}$

Wenn es sich bei den angeführten Rindern, welche in der $\mathbf{C h}$-Serie und auf Ce 50 und Ce 59 als Bos $^{\mathrm{m}}$ wiedergegeben sind, um Ochsen handelt, wäre es in Betracht zu ziehen, auch in den weiteren als Bos $^{\mathrm{m}}$ definierten Tieren der Linear B-Tafeln verschnittene männliche Rinder zu sehen. Desgleichen sind wohl auch unter dem Ideogramm sus ${ }^{\mathrm{m}}$ kastrierte männliche Schweine zu verstehen. Neben der Interpretation von we-ka-ta BOS $^{\mathrm{m}}$ und BOS ${ }^{\mathrm{m}} \mathrm{ZE}$ als Zeichen für Arbeitsochsen bieten die als ovis ${ }^{\mathrm{m}}$ gekennzeichneten Schafe, vor allem jene der knossischen D-Serien, einen weiteren Hinweis darauf, dass die Ideogramme für männliche Tiere, welche mit zwei horizontalen Strichen versehen wurden, Kastraten meinen. J. T. KILLEN hat durch seine Aufarbeitung der D-Serien feststellen können, dass es sich hierbei um Texte handelt, die sich mit Schafen beschäftigen, welche für die Produktion von Wolle als Rohstoff der Textilindustrie des Palastes von Knossos gehalten wurden. ${ }^{103}$ Auch hier sind unter dem Ideogramm ovIS ${ }^{\mathrm{m}}$ kastrierte männliche Tiere zu verstehen; Hammel liefern und lieferten mehr und bessere Wolle als weibliche Schafe und nicht kastrierte Schafsböcke. ${ }^{104}$ Auch das weniger häufig, aber doch vorkommende $\mathrm{CAP}^{\mathrm{m}}$ kann als Ideogramm für verschnittene Ziegenböcke gesehen werden. Zumindest ist aus der klassischen Antike bekannt, dass auch diese Wiederkäuer kastriert wurden. ${ }^{105}$ Durch die Kastration werden die männlichen Tiere beider Arten ruhiger, und der Geruch des Fleisches ist nicht mehr so intensiv, ${ }^{106}$ obwohl dieser, vor allem die ärmere Bevölkerung, nicht davon abhielt auch jenes unverschnittener Böcke zu essen. ${ }^{107}$ Gleichzeitig wird die Verfettung der Tiere gefördert, wodurch sie das zur Zeit Homers begehrte Fett und fette Fleisch ansetzten, ${ }^{108}$ welches wohl auch in mykenischer Zeit nicht verschmäht wurde. Die Nutzung des Fettes der kleinen Wiederkäuer zeigt sich an einem SH III B datierten Gefäß aus dem Südhaus von Mykene, in dem Bestandteile von Fett, welches von Schaf oder Ziege stammte, nachgewiesen werden konnte. ${ }^{109}$

Auch das Haarkleid der kastrierten männlichen Tiere wird feiner und lieferte das in der Antike genutzte Ziegenfell, aber auch die Woll- beziehungsweise

\footnotetext{
100 Ventris-Chadwick 1973, 213; Killen 1992-93, 101 u. Fn. 2; Palaima 1989, 89.

101 Ventris-Chadwick 1973, 213.

102 Siehe dazu S. 27ff.

103 Killen 1964, 1ff.; Killen 1968, 105ff.; Killen 1969, $23 f f$.

104 Killen 1964, 3f., 5; Killen 1969, 30f.

105 Keller 1909, 308.

${ }^{106}$ Im Dissertantenseminar des SS 2003 am Institut für Alte Geschichte und Altertumskunde der Universität Salzburg teilte mir Frau Mag. Caroline Dürauer mit, dass nach Auskunft des Landwirts Helmut Högler aus Seekirchen Ziegen heutzutage vorwiegend kastriert werden, um das Fleisch genießbarer zu machen.

107 Magerstedt 1859, $206 f$.

${ }^{108}$ Hom.Il.1,40f.; Hom.Od.2,56; 14,107; 20,186f.; 20,250.

109 Tzedakis-Martlew 1999, 134 Nr. 119.
} 
Filzverarbeitung aus Ziegenhaar war in der Antike durchaus bekannt. ${ }^{110}$ Nachdem in den Linear B-Texten der Cn-Serie aus Pylos neben Schafherden meiner Interpretation zufolge auch männliche verschnittene und weibliche Ziegen verzeichnet wären, ${ }^{111}$ könnte angenommen werden, dass das Haarkleid einiger ausgewählter Tiere auch in mykenischer Zeit Verwendung fand (vielleicht von Ziegen, deren Haar gut zur weiteren Verarbeitung geeignet war), beziehungsweise ist bei den weiblichen Tieren auch mit der Nutzung als Milchlieferant zu rechnen. In größerem Ausmaß wird die Verarbeitung von Ziegenhaar oder -fell aber nicht erfolgt sein, da dafür, sofern dies nicht an der Quellenlage liegt, zuwenige Ziegen beiderlei Geschlechts in den Linear B-Texten verzeichnet sind. Vor allem in den knossischen D-Serien, die sich explizit mit der Wollproduktion beschäftigen, sind die beiden Ideogramme $\mathrm{CAP}^{\mathrm{m}}$ und $\mathrm{CAP}^{\mathrm{f}}$ überhaupt nicht zu finden.

Die Kastration von männlichen Schafen und Ziegen hatte wohl auch den Zweck, die Zucht unter Kontrolle zu halten, da sich diese Tiere besonders stark vermehren. ${ }^{12}$ Generell war das Verschneiden, neben der getrennten Haltung von weiblichen und männlichen Tieren, eine Möglichkeit den Bestand von Tierherden unter Kontrolle zu halten. ${ }^{113}$

\subsection{INTERPRETATION DER IDEOGRAMME SUS, BOS, OVIS, CAP UND EQU}

Wenn alle Tiere, deren Ideogramme mit zwei horizontalen Strichen versehen sind - SUS $^{\mathrm{m}}$, BOS ${ }^{\mathrm{m}}$, OVIS ${ }^{\mathrm{m}}$, CAP ${ }^{\mathrm{m}}$ - als kastrierte männliche Tiere zu verstehen sind, stellt sich die Frage, unter welchen Zeichen die nicht verschnittenen Eber, Stiere, Schaf- und Ziegenböcke zu suchen sind. Bereits besprochen wurden die als sus $+K A$ bezeichneten Schweine der Tafeln PY Un 6.3.4, Un 853.5 und das als ta Bos definierte Rind von KN C(1) 901, doch können dies nicht die einzigen unversehrten männlichen Tiere gewesen sein. In diesem Fall bleibt nur mehr eine Kategorie von Ideogrammen zu untersuchen - jene ohne zusätzliches Determinativ, also die Ideogramme sus, BOS, OVIS und CAP.

\subsubsection{Interpretation als Jungtiere?}

Das Ideogramm sus ist auf den Tafeln PY Un 138.3 (sus 12), Cc 665 (sus 190) und auf der Tonplombe TH Wu 58. $\alpha$ zu finden. In Knossos ist es auf KN Ce 113, Co 903.2, C(3) 905, C(3) 979, C(3) 1030, C(3) 7057, C(3) 7058 vorhanden.

Neben dem bereits angeführten Mastschwein sind auf der Tafel Un 138 noch 12 Schweine unbestimmten Geschlechts (sus) verzeichnet. Mit aller Vor-

${ }^{110}$ Keller 1909, 302f.; auch in der Odyssee finden sich Belege für die Nutzung von Ziegenfell: Hom.Od.6,77 (Schlauch aus dem Fell einer Ziege); 14,524 (Ziegenfell als Kleidung); 16,47 (Geißfell als Sitzkissen); 14,51 (Haut einer wilden, bärtigen Ziege als Bettzeug).

111 CAP $^{f}$ auf PY Cn 45.6-.11, Cn 131.5.14, Cn 285.9, Cn 328.4.7, Cn 599.4-.6, Cn 600.12.36, Cn 643.6, Cn 702.3.4, Cn 1287.1-.10; $\mathrm{CAP}^{\mathrm{m}}$ auf $\mathrm{Cn}$ 45.12, Cn 201.2, Cn 202.5, Cn 285.3.4.6-.8, Cn 328.3.6.14, Cn 436.2, Cn 599.1.2, Cn 643.3.5, Cn 1069.6.

112 Magerstedt 1859, 205f: „Der Geschlechtstrieb erwacht bei Allen sehr früh; die schalkhaften Böckchen und Böcke dienen im Cultus, gleich den Ziegen, wegen ihrer zeugungslustigen Natur, als Opfer der Venus, der Vorsteherin der Zeugungen (Plaut. Poenul. II.1. Virg. Aen. I. 419)“; S. 210: „Die kurze Trachtzeit macht möglich, dass Manche jährlich zwei Mal werfen (Virg. E. I. 10. III. 34 VII. i G. II. 150) und also vom dritten bis zum achten Jahre zwei Lämmer angenommen, vierundzwanzig Junge liefern kann.“

113 Benecke 1994, 175. 
sicht könnte man annehmen, dass diese 12 Schweine Ferkel waren, da in der pylischen U-Serie sonst weibliche Tiere $\left(\mathrm{sus}^{\mathrm{f}}\right)$, Mastschweine (suS $+S I$ ) und Zuchteber (sus $+K A$ ) ausgewiesen sind. Die Interpretation als Ferkel geht davon aus, dass bei sehr jungen Tieren das Geschlecht für bestimmte Zwecke nicht von allzu großer Bedeutung war. Für die Schlachtung zum Verzehr bei kultischen Gelegenheiten war der Unterschied zwischen männlichen und weiblichen Jungtieren nicht erheblich, da es vorwiegend um den Fleischansatz ging. ${ }^{114}$ Aber auch bei Formen von Opfern, bei denen das Fleisch für die Opfernden tabu war und nicht verzehrt wurde (Beispiel für Eid-, Sühne- und Totenopfer sowie als Gabe für chthonische Gottheiten) ${ }^{115}$, scheint das Geschlecht der Tiere nicht maßgeblich gewesen zu sein.

Ferkel waren gängige Opfertiere im Kult. So ist in der Naturalis Historia des älteren Plinius zu lesen, dass Ferkel schon am fünften Tage zum Opfer „rein“ sind. ${ }^{116}$ Die Jungtiere fanden besonders als Reinigungsopfer Verwendung, so wurden zum Beispiel das athenische Volk bei Volks- und Festversammlungen durch Ferkelopfer gereinigt, und in Andonia (Messenien) reinigte ein Priester das Theater durch das Opfer von drei Ferkeln. ${ }^{117}$ Für die Göttin Demeter Potnia in Potniai bei Theben wurden Ferkel zur Bestrafung in einer Erdkluft versenkt, da sie dem Mythos zufolge die Fußspuren der von ihrer Mutter gesuchten Persephone zertreten hatten. ${ }^{118}$

Es besteht jedoch auch die Möglichkeit, dass die genauere Klassifizierung der Schweine als „männlich“ oder ,weiblich“ vom Schreiber mit Absicht weggelassen worden ist. In beiden Fällen (Ferkel oder erwachsene Tiere) war das Geschlecht damit anscheinend nicht von Bedeutung. Es ist freilich auch möglich, dass der Schreiber eine genaue Definition vergaß. Immerhin findet sich auf der Tafel PY Ua 17.2 das Ideogramm sus ${ }^{f}$ mit der Zahl 20. Dies ist eine ähnlich beachtliche Anzahl - hier allerdings an Sauen - wie jene als sus definierten Schweine auf Un 138. Desgleichen findet sich auf KN Co 903.2 das Ideogramm sus angeführt. In Hinblick auf die noch zu besprechenden Texte, vor allem KN C 902 und PY Cn 3, die das Ideogramm Bos enthalten, stellt sich jedoch die Frage, ob unter sus männliche, nicht kastrierte Tiere zu sehen sind.

Als Argument gegen die Deutung des Ideogrammes sus als Kennzeichnung von Ferkeln ist anzuführen, dass zumindest bei den Schafen der knossischen D-Serien zusätzliche Silbenzeichen $\left(k_{i}\right.$ ovIS $^{\mathrm{m}}$ in der KN Dl-Serie, $k i$ ne ovIS ${ }^{\mathrm{m}}$ auf KN Dk 1066.A, Dk 1967.A) die jungen Tiere genauer charakterisieren. ${ }^{119}$ Auch das Zeichen WE/we (*wetalon - „Jährling“) in Knossos, Pylos und Theben ${ }^{120}$ wird in Zusammenhang mit Schafen und Ziegen als Ausdruck für ein Jungtier verwendet. ${ }^{121}$ Doch nicht nur bei Schafen und Ziegen wurden die jungen Tiere derart gekennzeichnet. Auf der Tafel KN Ca 985 + fr. sind Pferde (i-qo in Zeile .1) und Esel (o-no in Zeile .2) verzeichnet. Dabei wurden von den

\footnotetext{
114 Siehe S. 10f.

115 Siehe dazu Stengel 1910, 92ff., 126ff.; Ziehen 1939, 622.

116 Plin.nat.8,77,206.

117 Orth 1921, 812.

${ }^{118}$ Gruppe 1906, 38 und Fn. 6.

119 Killen 1964, 12; Killen 1968, 113f., 121f.; Piteros-Olivier-Melena 1990, 161 Fn. 237.

${ }^{120}$ KN C 7060 + fr. [+] 8791.1, Dl 790.A, Dl 791.A, Dl 916.A, Dl 934.A, Dl 7072.A, Dl 7076.A, Dl 7086.A, Dl 7092.A, D 411 + 511; PY Cn 418.4.8, Ua 17.2, Un 47.5, Un 138.3, Un 1185.4; TH Wu 64. $\alpha$, Wu 74. $\alpha$, Wu 78. $\beta$ ).

${ }^{121}$ Siehe S. 25; Ventris-Chadwick 1973, 590; Piteros-Olivier-Melena 1990, 161; KN C 7060 + fr. [+] 8791.1, Dl 790.A, Dl 791.A, Dl 916.A, Dl 934.A, Dl 7072.A, Dl 7076.A, Dl 7086.A, Dl 7092.A, D 411 + 511; PY Cn 418.4.8, Ua 17.2, Un 47.5, Un 138.3, Un 1185.4; TH Wu 64. $\alpha$, Wu 74. $\alpha$, Wu 78. $\beta$.
} 
Paarhufern jeweils die Ideogramme EQU ${ }^{\mathrm{m}}$ (männliches Pferd) und EQU ${ }^{\mathrm{f}}$ (weibliches Pferd) aufgelistet und zusätzlich das Ideogramm EQU mit dem Wort po-ro vorgestellt, welches dem klassisch-griechischen $\pi \tilde{\omega} \lambda$ o „Fohlen“ entspricht. ${ }^{122}$ Hier zeigt sich, dass das Jungtier nicht allein durch das Ideogramm ohne horizontale Striche gekennzeichnet wurde, sondern wiederum durch einen Zusatz, diesmal einem ganzen Wort, po-ro. Auf Grund dieser Beispiele kommt die Interpretation als junges Tier für das bloße sus, aber auch BOs, OVIS, CAP und EQU nicht in Frage.

\subsubsection{Interpretation als kastrierte Tiere}

Das Ideogramm Bos ist auf den Tafeln PY Cn 3.3-.7 und Un 2.5 zu finden, sowie in Knossos auf Ce 59.2b, Ce 61.2-.4, Ce 144.1, Ce 152.2.3 v.1-.3, Ce 156.1.2, Ce 7061.1.2, Ch 7047, Ch 7066, Ch 7100, C 902.1-.12, C 5734 und C 7698.

Th. Palaima schrieb 1989 in seinem Artikel Perspectives on the Pylos Oxen Tablets über die als Bos ${ }^{\mathrm{m}}$ und Bos definierten Rinder: „,..., the tablet Ce 59 refers to animals identified as we-ka-ta, i.e., ,workers, “ five times as BOs ${ }^{\mathrm{m}}$ and once as plain Bos. Is this distinction meaningful? Are five of the groups male animals destined for worker status and therefore to be castrated, whereas animals of the sixth group already have been neutered? Is the generic form of the sixth group - actually the fourth group entered on the tablet - a scribal omission, oversight, or bit of carelessness?" ${ }^{\text {123 }}$ Diese Ausführung ist auch auf die in der $\mathbf{C h}$-Serie von Knossos vorkommenden Rinder beziehungsweise deren Ideogramme BOS $^{\mathrm{m}}$ $Z E$ und BOs $Z E$ anwendbar.

Die Vermutung, dass es sich bei we-ka-ta Bos auf der Tafel C 59.2b um eine Verschreibung für we-ka-ta BOS $^{\mathrm{m}}$ handeln kann, ist durchaus in Betracht zu ziehen. Wird jedoch das Ideogramm Bos ZE der weiteren Texte der Ce- und ChSerien (KN Ce 59.2b, Ce 144.1, Ce 7061.1.2, Ch 7047, Ch 7066 und Ch 7100 + 7703), welches ebenfalls als Paare von Arbeitstieren gesehen werden darf, auf einen möglichen Schreibfehler hin überprüft, liefe dies auf eine relativ hohe Fehlerquote der Schreiber 124 und 110 hinaus. So zeigt das Verhältnis von Bos der genannten Tafeln zu BOS ${ }^{\mathrm{m}}$ (KN Ch 896, Ch 897, Ch 898, Ch 899, Ch 900, Ch 1015, Ch 1029, Ch 5724, Ch 5728, Ch 5938, Ch 7065) einen relativ hohen Anteil an BOs (sechsmal BOS ZE zu elfmal BOS ${ }^{\mathrm{m}} \mathrm{ZE}$ ). So sollte berücksichtigt werden, dass es sich nicht um einen Fehler der Palastschreiber (Hand 124 und Hand 110) gehandelt haben muss, sondern dass diese aufgrund der Tatsache,

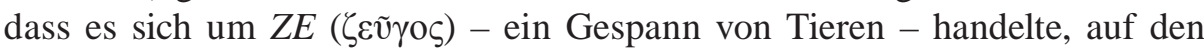
genauen Definitionsunterschied von Bos (unverschnittenen Rindern) und Bos ${ }^{\mathrm{m}}$ (verschnittenen Rindern) kein besonderes Augenmerk legten. Das heißt, dass ihnen (und der Palastverwaltung) klar war, dass es sich bei mit $Z E$ spezifizierten Tieren um kastrierte Ochsen handeln musste. Immerhin wurde das Wort ßoṽ bei Homer und in klassischer Zeit generell für Rinder verwendet und konnte sowohl den Bullen als auch die Kuh und den Ochsen bezeichnen. Auch in mykenischer Zeit war dieses Wort, dessen Stamm ${ }^{*} \gamma^{\mathrm{w}}$ oṽ- wir aus Wörtern wie qo-o oder qo-u-ko-ro kennen, in Gebrauch. ${ }^{124}$ Die Schreiber verzeichneten

122 Ventris-Chadwick 1973, 573.

123 Palaima 1989, $91 \mathrm{f}$.

${ }^{124}$ Der Stamm ${ }^{*} \gamma^{w}$ ov - findet sich als qo-o $\left({ }^{*} \gamma^{w} \tilde{\omega} v \varsigma\right)$ auf PY Cn 3.2, als qo-u-ko-ro/-jo (* $\left.\gamma^{\mathrm{w} o v \kappa o ́} \lambda \circ \varsigma\right)$ auf TI Ef 2, PY An 18.9, An 830.10.11.12.13, An 852.1, Nn 831.5, als qo- 
somit die gesprochenen Wörter „Arbeitsrind“ und „Rindergespann“ mit dem Ideogramm *109, welches als ßoṽ $\left({ }^{*} \gamma^{\mathrm{w}} \mathrm{ov} \varsigma\right.$ ) zu deuten ist, und verwendeten sowohl die einfache Form Bos als auch das, nach meiner Interpretation, kastrierte Tiere definierende BOs $^{\mathrm{m}}$.

Damit komme ich auf das Zitat TH. Palaimas zurück, ${ }^{125}$ in dem er eine Unterteilung in bereits kastrierte und noch zu verschneidende Arbeitstiere vornahm. Er ging dabei davon aus, dass die als Bos bezeichneten Rinder bereits verschnitten waren, während die BOS $^{\mathrm{m}}$ geschriebenen Tiere noch dieser Prozedur zu unterziehen gewesen wären. Ich kann mich dieser Unterteilung insoweit anschließen, dass unter den genannten Tieren kastrierte und nicht kastrierte Rinder zu finden sind. Allerdings möchte ich aus den noch zu nennenden Gründen das Ideogramm Bos ${ }^{\mathrm{m}}$ als Zeichen für verschnittene und das bloße BOs als jenes für unverschnittene Tiere sehen. Bereits M. VENTRIs und J. CHADWICK sprachen in Documents in Mycenaean Greek die als we-ka-ta BOS $^{\mathrm{m}}$ bezeichneten Tiere als kastrierte Rinder an. ${ }^{126}$ Dies dadurch, dass auch in

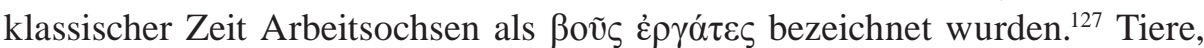
deren Ideogramme mit horizontalen Strichen versehen wurden, als kastriertes männliches Vieh zu sehen, bietet sich durch den Vergleich mit dem Zeichen ovIS $^{\mathrm{m}}$ der knossischen D-Serien an. Hier sei nochmals auf die Aufarbeitung J. T. KILLENS verwiesen, in der er nachweisen konnte, dass es sich bei den in diesen Serien als ovis ${ }^{\mathrm{m}}$ bezeichneten Schafen, um kastrierte männliche Tiere gehandelt haben muss. ${ }^{128}$

Durch die Nutzung der Rinder als Arbeitstiere und die Parallele zu den Schafen, die in der Wollproduktion Verwendung fanden, darf angenommen werden, dass BOS $^{\mathrm{m}}$ generell als Ideogramm für kastrierte männliche Rinder zu verstehen ist. Es wäre jedoch widersinnig, die als Bos bezeichneten Tiere ebenfalls als verschnittene Tiere anzusehen. ${ }^{129}$ Dies gilt natürlich auch für die sus/ SUS $^{\mathrm{m}}$, OVIS/OVIS ${ }^{\mathrm{m}}$ und CAP/CAP ${ }^{\mathrm{m}}$ geschriebenen Schweine, Schafe und Ziegen. Eine Ausnahme bildet natürlich das Ideogramm ovs ${ }^{\mathrm{m}}$ der knossischen Da- und Dg-Serien. Wie J. T. KILLEN nachweisen konnte, wurden hierbei sowohl männliche als auch weibliche Tiere zusammengefasst. ${ }^{130}$ Dies ändert jedoch nichts daran, dass die männlichen Tiere verschnittene Hammel waren.

\subsubsection{Interpretation als unkastrierte Tiere}

Wenn meiner Interpretation zufolge alle Tiere, deren Ideogramme mit zwei horizontalen Strichen versehen sind $\left(\right.$ BOS $^{\mathrm{m}}$, SUS $^{\mathrm{m}}$, ovIS ${ }^{\mathrm{m}}$, CAP $\left.{ }^{\mathrm{m}}\right)$, kastrierte männliche Tiere waren, wäre die Lösung der anscheinend widersprüchlichen Angaben von BOS $^{\mathrm{m}} \mathrm{zu}$ BOS - sowie sUS ${ }^{\mathrm{m}} \mathrm{zu}$ SUS, OVIS ${ }^{\mathrm{m}} \mathrm{zu}$ OVIS und CAP ${ }^{\mathrm{m}} \mathrm{zu}$ CAP -, in den Tierideogrammen ohne horizontale Striche generell männliche, nicht kastrierte Tiere zu sehen. Nicht zu leugnen ist, dass in manchen Fällen eine Verschreibung vorliegen kann.

qo-ta-o $\left({ }^{*} \gamma^{\mathrm{w}} \mathrm{O}(v) \gamma^{\mathrm{w}} \mathrm{o} \tau \alpha \varsigma\right)$ auf PY Ea 270, Ea 305.a, Ea 757.a, Ea 802.a, als qo-u-qo-ta

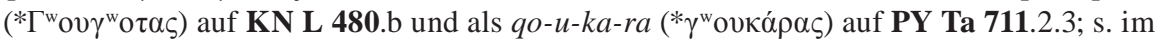
Allgemeinen dazu Aura Jorro 1993, s.v. qo-o, qo-qo-ta-o, qo-u-ko-ro, qo-u-qo-ta.

125 Siehe S. 25 u. Fn. 123.

126 Ventris-Chadwick 1973, 213.

127 Ventris-Chadwick 1973, 590; Liddell-Scott 1996, s.v. غ̇ $\rho \gamma \alpha \tau \varepsilon i ́ \alpha$.

${ }^{128}$ Siehe S. 22 u. Fn. 103, 104.

${ }^{129}$ Siehe dazu den folgenden Abschnitt S. 27ff.

130 Killen 1964, $10 \mathrm{ff}$. 
Zwei Tierideogramme der Linear B-Texte belegen auf hervorragende Weise den Gebrauch von einfachen, nicht näher definierten Ideogrammen (ohne horizontale Striche) für männliche, unverschnittene Tiere. Dies sind die Zeichen EQU/EQU ${ }^{\mathrm{m}}$ der Tafel KN Ca 895 + fr.1.2 und CERV der zwei pylischen Tafeln PY Cr 591.1-.7, Cr 868 [+] Cr 875.1-.7131.

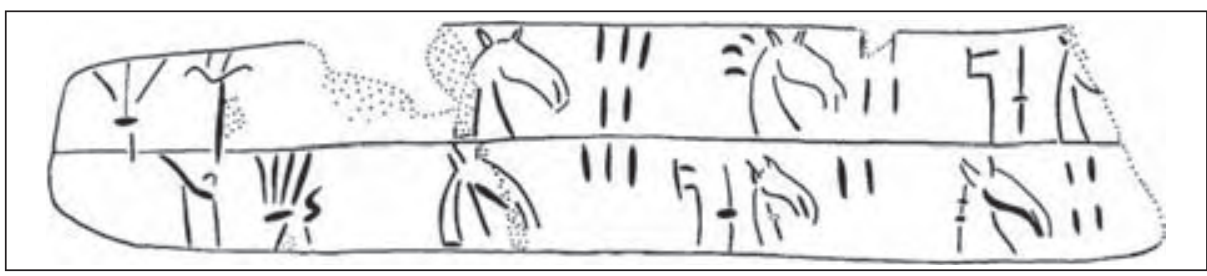

Tab. 3: KN Ca 895 + fr.1.2: Ideogramm *105 EQUm (nach Chadwick-Godart-Killen 1986, 363).

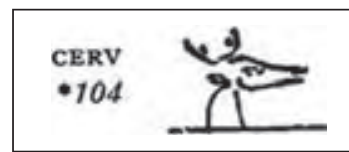

Tab. 4: PY Cr-Serie Ideogramm *104 CERV (aus Bennett-Olivier 1973, 82).

Zunächst zum Text von KN Ca 895, der in Zeile .1 die Angabe i-qo (i $\pi \pi \circ$ ) „Pferde“, enthält (Tab. 3). Darauf folgt das Ideogramm EQUf, mit dem für weibliche Tiere charakteristischen zusätzlichen vertikalen Strich und der Angabe von fünf Stück. Im Anschluss findet sich ein Pferdekopf dargestellt, welcher mit drei Strichen, die eine Mähne andeuten, versehen ist und generell als EQU ${ }^{m}$ gedeutet wird. Dieses wird als das Ideogramm für ein männliches Pferd angesehen, wovon vier Tiere verzeichnet sind. In derselben Zeile folgt das Wort po-ro $(\pi \tilde{\omega} \lambda \mathrm{o})$ ),Fohlen“, und das Ideogramm, diesmal ohne weitere Striche zur genauen Angabe des Tieres, also EQU geschrieben. Die Zeile .2 enthält die Angabe $o$-no (övol) „Esel“, und es folgt wiederum das Ideogramm EQU ${ }^{\mathrm{f}}$ für das weibliche Tier (drei Stück), dann das Wort po-ro mit dem bloßen Ideogramm EQU (zwei Fohlen) und das Ideogramm - diesmal mit zwei horizontalen Strichen eingeschrieben - $\mathrm{EQU}^{\mathrm{m}}$, insgesamt vier männliche Esel.

Die Ideogramme der weiblichen Tiere sind eindeutig und bedürfen keiner näheren Besprechung. Interessant im Zusammenhang mit der Interpretation der Ideogramme ohne horizontale Striche sind die Zeichen für Fohlen und männlichen Pferde sowie Esel. Für die Angabe von Fohlen wurde sowohl bei den Pferden als auch bei den Eseln das einfache Ideogramm EQU benutzt. Jedoch reichte dies offensichtlich nicht aus, um die Tiere als Jungtiere zu charakterisieren, weshalb in beiden Fällen zusätzlich das Wort po-ro hinzugefügt wurde. Auch scheinen die Köpfe etwas kleiner gezeichnet worden zu sein als jene der erwachsenen Tiere. Das Ideogramm für die Fohlen selbst zeigt, bedingt durch das Fehlen eines zusätzlichen vertikalen Striches, lediglich an, dass es sich um männliche Tiere handelt. Durch die nicht vorhandenen horizontalen Striche darf angenommen werden, dass hierbei nicht kastrierte Pferde- und Eselfohlen gemeint waren. Diese Interpretation wird durch die Darstellung der Ideogramme für die erwachsenen männlichen Tiere angeregt. In den Publikationen der Knossos Tafeln (The Knossos Tablets und Corpus of Mycenaean Inscriptions from Knossos) wurde zu den männlichen Pferden der Zeile .1 angemerkt: ,..1 $\mathrm{EQU}^{\mathrm{m}}$ is here shown by the mane instead of the usual two cross-

\footnotetext{
131 Join nach Melena 1994-95, 284.
} 
bars as in line 2“.132 Die Darstellungsweise des Ideogrammes für männliche Pferde mit Mähne in Ca 895.1 gegenüber den maskulinen Eseln in Zeile .2 mit den horizontalen Strichen muss als bewusste Unterscheidung des Palastschreibers gesehen werden. So findet sich das Ideogramm ohne horizontale Striche, aber als Pferdekopf mit Strichen, die eine Mähne angeben, auch in der knossischen Sc-Serie, wo Gespanne von Pferden (EQU ZE) oder einzelne Pferde (EQU MO) in Zusammenhang mit der Ausrüstung für Streitwagen angegeben sind. ${ }^{133}$ Aus der klassischen Zeit ist bekannt, dass männliche Pferde generell nicht kastriert wurden, um ihr ungestümes, schnelles Naturell für den Einsatz bei Streit- und Rennwagen zu nutzen. ${ }^{134}$ Im Gegensatz dazu wurden die als Last- und Zugtiere eingesetzten männlichen Esel zwar nicht generell, aber doch kastriert, wodurch sie folgsamer und leichter zu handhaben waren. ${ }^{135}$ Wir dürfen annehmen, dass in mykenischer Zeit in gleicher Weise mit den beiden Tiergattungen verfahren wurde. Es ist deshalb nicht verwunderlich, dass auf der Tafel Ca 895.2 das Ideogramm EQU ${ }^{\mathrm{m}}$ für den männlichen Esel Verwendung fand. Das zusätzliche Angeben der Mähne auf Ca $\mathbf{8 9 5 . 1}$ und in der Sc-Serie dürfte auf die Qualität des Pferdes hinweisen, vor allem wenn man bedenkt, dass in der klassischen Zeit unter anderem eine schön ausgeprägte Mähne bei den männlichen Pferden als Ausweis der hervorragenden Eigenschaften eines Pferdes - besonders für den Kriegsdienst - galt. ${ }^{136}$ Es zeigt sich die bewusste Unterscheidung von männlichen, unverschnittenen Pferden und verschnittenen Eseln. Als Konsequenz der Interpretation des männlichen Pferdeideogrammes von Ca 895.1 müsste eine Umschreibung der Transliteration von EQU ${ }^{\mathrm{m}}$ auf EQU erfolgen, was dem Zeichen EQU der Sc-Serie entspräche.

In den Linear B-Texten findet sich des Weiteren eine Tiergattung, an dessen Ideogramm sehr deutlich zu erkennen ist, dass das Zeichen für männliche, nicht kastrierte Tiere ohne horizontale Striche auskam. Dies ist das Ideogramm CERV (Tab. 4) der zwei Tafeln PY Cr 591.1-.7, Cr 868 [+] Cr 875.1-.7137. Ohne Zweifel ist der dargestellte Tierkopf, ausgehend von dem angegebenen Geweih, einem Hirschen zuzurechnen. ${ }^{138}$ Bei dieser Tiergattung tragen ausnahmslos nur die Männchen ein solches. ${ }^{139}$ Es darf mit Sicherheit angenommen werden, dass es sich bei den angeführten Tieren um männliche und unverschnittene Hirsche

132 J. T. Killen u. J.-P. Olivier: The Knossos Tablets. Suplementos a MINOS Núm. 11. Salamanca 19895; J. Chadwick, L. Godart, J. T. Killen u. a.: Corpus of Mycenaean Inscriptions from Knossos. Vol. I, Rom 1986; Vol. II, Rom 1990; Vol. III, Rom 1997, Vol. IV Rom 1998.

133 Plath 1994, 107.

134 Keller 1909, 237; kastrierte männliche Pferde (Wallachen) scheinen erst in römischer Zeit bekannt zu werden - s. dazu Keller 1909, 237; Steier 1938, 1433; Varro rust.2,7,15: „Da die einen von ihnen sich zum Heereseinsatz eignen, die anderen aber zum Fuhrbetrieb, zur Zucht oder zum Wettrennen, darf man sie nicht einheitlich betrachten und halten. Dem entspricht, dass der kriegserfahrene Stallmeister andere auswählt, aufzieht und abrichtet, anders der Viergespannlenker und der Kunstreiter verfahren und sich genausowenig für dieselbe Vorgehensweise entscheidet, wer Pferde, die zur Fortbewegung dienen sollen, auf die Schabracke oder auf die Reisekutsche vorbereiten will. Denn wie man für den Heereseinsatz feurige haben möchte, weil sie dort für das Feldlager bestimmt sind, so will man umgekehrt auf den Straßen lieber zahme haben. Wegen dieses Unterschieds vor allem hat es sich eingebürgert, Hengste zu verschneiden.“

135 Keller 1909, 267; Olck 1907, 637.

136 Keller 1909, 235f.; Colum.6,29,2ff.

137 Join nach Melena 1994-95, 284.

138 Ventris-Chadwick 1973, 195: „The signs for the HORSE and the DEER are self-evident“.

139 Barth 1953, 564: „In der Familie der Hirsche (Cervidae) werden alle geweihtragenden Wiederkäuer zusammengefaßt. [...] Die Geweihe kommen mit einer Ausnahme (Renntier) nur den Männchen zu.“ 
gehandelt hat. Bemerkenswert ist dabei, dass die Darstellung des Hirschkopfes in der pylischen $\mathbf{C r}$-Serie immer ohne Zufügung von horizontalen Strichen erfolgte.

Somit weisen die Ideogramme EQU für männliche Pferde und CERV für männliche Hirsche, die mit Sicherheit unverschnittene Tiere darstellen, keine zwei horizontalen Striche auf. Durch diese Beispiele ist anzunehmen, dass auch die weiteren Tierideogramme BOS, sUs, OVIS und CAP ebenfalls nicht kastrierte männliche Rinder (Stiere), Schweine (Eber), Schafe und Ziegen (Schafund Ziegenböcke) wiedergeben sollen. Diese Annahme wird durch das geringe Auftreten der bloßen Ideogramme ovIS, CAP, sus und Bos im Vergleich zu jenen mit zwei horizontalen Strichen OVIS ${ }^{\mathrm{m}}$, CAP $^{\mathrm{m}}$, SUS $^{\mathrm{m}}$ und BOS ${ }^{\mathrm{m}}$ noch verstärkt. Nachdem anzunehmen ist, dass in der mykenischen Zeit, wie zu allen Epochen, die meisten männlichen Tiere der Schafe, Ziegen, Schweine und Rinder verschnitten wurden, wird dies verständlich. Warum sollten die Schreiber der mykenischen Palastverwaltung eine Unterscheidung zwischen weiblichen, jungen, gemästeten und speziellen Zuchttieren vorgenommen, für verschnittene und unverschnittene Tiere jedoch dasselbe Ideogramm mit horizontalen Strichen verwendet haben?

Auffällig ist zusätzlich, dass bei den Auflistungen von Rindern in den Linear B-Texten, das einfache Ideogramm Bos auf Texten zu finden ist, deren Aufzeichnungen über den alltäglichen Gebrauch von Arbeitstieren hinausgehen. So ist Bos auf den Tafeln PY Cn 3.3-.7, Un 2.5 und KN C 902.1-.12 verzeichnet. Der Inhalt von Un 2 wurde bereits weiter oben durchgenommen, ${ }^{140}$ doch bleibt hier zu sagen, dass die angeführten Tiere, darunter ein männliches unkastriertes Rind, und pflanzliche Güter für eine Feierlichkeit in Zusammenhang mit der Initiation des wanaks benötigt wurden. Im Folgenden sollen die beiden Tafeln PY Cn 3 und KN C 902 besprochen werden.

PY

Cn 3

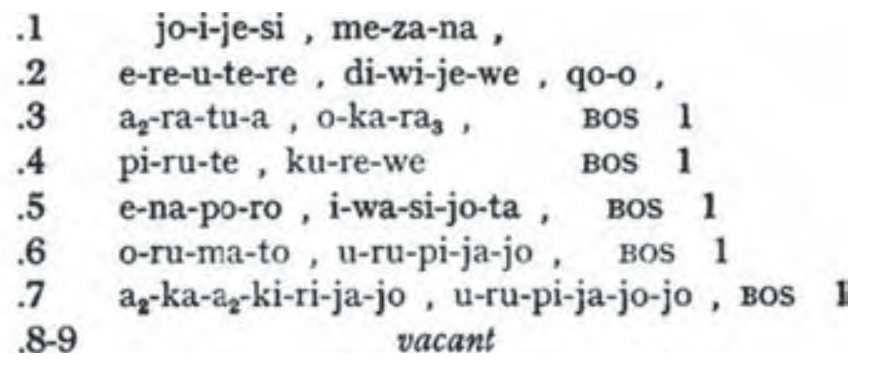

S608 - H1

Die Tafel PY Cn 3 weist drei Gemeinsamkeiten zur bereits besprochenen Tafel PY Cn 608 auf. So wurden zum einen beide Tafeln von der Schreiberhand $\mathrm{H} 1$ - S 608 verfasst und zum anderen ist auf beiden Texten jeweils nur eine Kategorie von Tieren aufgelistet. In den Zeilen Cn 3.3-.7 ist jeweils ein Rind (Bos) verzeichnet, während die Zeilen Cn 608.3-11 ausschließlich Mastschweine (sus $+S I$ ), allerdings in unterschiedlicher Anzahl, ${ }^{141}$ aufweisen. Der dritte gemeinsame Punkt der beiden Tafeln betrifft die Angabe der „Lieferanten“; nach den Aussagen beider Tafeln waren keine Einzelpersonen zur Lieferung verpflichtet, sondern Gemeinschaften. Nach Cn 608 wurden die Einwohner einzelner Gemeinden der Diesseitigen Provinz mit der Mästung und damit verbunden wohl auch der Lieferung der Mastschweine beauftragt. ${ }^{142}$ Der

\footnotetext{
140 Siehe S. 8f.

141 Siehe S. 6.

142 Siehe S. 7 u. Fn. 11.
} 
Text von Cn 3.3-.7 nennt ebenfalls Toponyme - Cn 3.3 $a_{2}$-ra-tu-a, .4 pi-ru-te,

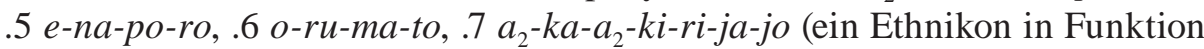
eines Toponyms), zusätzlich sind einzelne Personengruppen - Cn 3.3 o-ka$r a_{3}, .4$ ku-re-we, . 5 i-wa-si-jo-ta, .6.7 u-ru-pi-ja-jo(-jo) - angegeben. Dieselben Bezeichnungen und Ortsnamen finden sich auch in der An-Serie von Pylos, in den so genannten o-ka-Texten (o-ka-ra $a_{3} a_{2}-r a-t u-a$ auf PY An 519.4, pi-rute ku-re-we auf An 519.14, e-na-po-ro i-wa-si-o auf An 661.3, u-ru-pi-ja-jo o-ru-ma-si-ja-jo auf An 519.11.12 und $a_{2}-k a-a_{2}-k i-r i-j o ~ u-r u-p i-j a-j o$ auf An 661.12). ${ }^{143}$ Wie diesen Tafeln zu entnehmen ist, gehören sie den militärischen Verbänden des Reiches von Pylos an, und dieselben Truppen sollten im Falle von Cn 3 Stiere an eine Person di-wi-je-u (Zeile .2) senden (Zeile .1 jo-i-je-si: „so sollen sie senden“). ${ }^{144}$ Dieser di-wi-je-u könnte wiederum in der An-Serie von Pylos zu finden sein, wo er auf PY An 656.8.9 (ebenfalls ein $o-k a$-Text) als $e$-qe-ta definiert ist. ${ }^{145} \mathrm{Er}$ hätte demnach ebenfalls eine militärische Funktion, allerdings wohl auf höherer Ebene als die zuvor genannten Truppenverbände. Damit schiene es nicht verwunderlich, wenn die in Cn 3.3-7 aufgelisteten Mannschaften je einen Stier an di-wi-je-u sandten.

Unklar ist jedoch die genaue Auslegung der Funktion dieses Mannes, der als e-qe-ta eindeutig mit militärischen Funktionen ausgestattet war. Das Wort di-wi-je- $u$ wurde sowohl als Amtstitel („Priester des Zeus“) als auch als Personenname gedeutet; ${ }^{146} \mathrm{~S}$. DEGER-JALKOTZY sprach sich unter Bezugnahme auf moderne Berufsbezeichnungen (z. B. Doktor), die sich der Funktion von Eigennamen annähern, für erstere Auslegung aus. ${ }^{147}$ In Documents in Mycenaean Greek dagegen wird die Verwendung von di-wi-je- $u$ als Eigenname nicht ausgeschlossen. ${ }^{148}$ Beide Interpretationen sind in Bezug auf die zu liefernden Stiere durchaus in Erwägung zu ziehen; einmal ein Zeuspriester (gleichzeitig e-qeta), der für kultische Belange in Zusammenhang mit militärischen Operationen zuständig war, zum anderen ein hoher militärischer Funktionär (e-qe-ta), der die Verantwortung für die an ihn übergebenen Tiere hatte. In beiden Fällen ist eine Lieferung der Rinder für einen besonderen Zweck - vor allem im kultischen Sinne - anzunehmen. Diese Interpretation ergibt sich aufgrund der Tatsache, dass es sich: 1.) um unverschnittene männliche Rinder (BOS) ${ }^{149}$ handelte, 2.) jede angeführte Mannschaft nur jeweils ein Tier zu stellen hatte und 3.) zusätzlich eine Verbindung - durch den Schreiber und die Verpflichtung - zur Tafel PY Cn 608 zu erkennen ist. Beide Texte (Cn 3 und Cn 608) wurden von derselben Schreiberhand H 1 - S 608 verfasst und enthalten, wie bereits angesprochen, Angaben über zu leistende Verpflichtungen von Gemeinschaften beziehungsweise Personengruppen. So sollten nach Cn 3.1.2 unterschiedliche Truppenverbände Rinder an di-wi-je- $u$ liefern, während auf $\mathbf{C n} 608$ einzelne Gemeinden der Diesseitigen Provinz aufgefordert waren, Schweine zu mästen.

\footnotetext{
143 Zu den $o$-ka-Texten gehören neben den genannten Tafeln PY An 519 und An 661 noch die

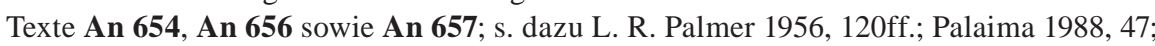
Uchitel 1984, 136ff., 156; Lindgren 1973 II, 44 u. Fn. 2; Aura Jorro 1993, s.v. o-ka.

144 Deger-Jalkotzy 1978, 19; Palaima 1989, 104.

${ }^{145}$ Für die Gleichsetzung des di-wi-je-u von PY Cn 3.2 zu jenem von PY An 656.9 s. Lindgren 1973 I, 42.

146 Ruijgh 1999, 535 Fn. 535 („„chef du sanctuaire de Zeus’(nom propre?)“); Leukart 1994, 176 („,Zeuspriester“); Aura Jorro 1985, s.v. di-wi-je-u („«servidor del santuario de Zeus» - mejor que «sacerdote de Zeus»"); L. R. Palmer 1963, 174.

147 Deger-Jalkotzy 1978, 19 u. Fn. 42; ähnl. Ilievski 1999, 302f., 308, 311.

148 Ventris-Chadwick 1973, 207, 435, 540; Lindgren 1973 II, 51.

149 Zur Interpretation des Ideogrammes Bos als Zeichen für unverschnittene Tiere s. $27 \mathrm{ff}$.
} 
Letztere wurden bereits als Tiere interpretiert, die wohl für eine besondere Gelegenheit gedacht waren; ${ }^{150}$ dies scheint auch für die sieben Rinder der Tafel Cn 3 zuzutreffen. ${ }^{151}$

Ob es sich bei den Rindern um Opfer für den Gott Zeus beziehungsweise ein Zeusheiligtum handelte, hängt nun von der Interpretation des Wortes di$w i-j e-u$ ab. Wenn wir von der Deutung als „Priester des Zeus“ ausgehen, ist eine Lieferung von Opfertieren für den Kult des Gottes Zeus nicht zu leugnen. Sollte di-wi-je-u jedoch ein Personenname sein, lässt sich diese Verbindung nicht mehr ohne weiteres feststellen. Eine Lösung des Problems (Titel oder Personenname) wäre durch die Interpretation von S. DEGER-JALKOTZY gegeben: „... sie sehen in di-wi-je- $u$ lediglich einen Eigennamen. Die Grenzen können in einem solchen Fall freilich verschwimmen. So werden in manchen ländlichen Gebieten Österreichs Berufsbezeichnungen wie „der Pfarrer“, „der Apotheker“, „der Doktor“ (= der Arzt) durchaus auf ganz bestimmte Personen bezogen und nähern sich damit der Funktion des Eigennamens. “152 Es braucht nicht zu verwundern, wenn ein militärischer Funktionär (e-qe-ta) auch eine Aufgabe im Kult zu erfüllen hätte. ${ }^{153}$ Als Parallele dazu könnte der mit di-wi-je- $u$ in der PY Es-Serie vorkommende we-da-ne-u dienen. ${ }^{154}$ Letzterer hatte offensichtlich ebenfalls kultische Verpflichtungen zu erfüllen, wie durch die Tafel PY Cn 418 zu ersehen ist. ${ }^{155}$ Beide, di-wi-je- $u$ und we-da-ne- $u$, sind in der Es-Serie neben Poseidon als Empfänger von Getreide genannt und damit wiederum in den kultischen Bereich eingegliedert. ${ }^{156}$ Dass es sich bei dem e-qe-ta di-wi-je-u um eine höhere Persönlichkeit in der Hierarchie (auch der e-qe-ta) handelte, wird gerade durch die Aufzeichnungen der Es-Serie bestätigt. Auf der Tafel Es 649.1 wird ein weiterer e-qe-ta, a-re-ku-tu-ru-wo (welcher auf PY An 654 als e-qe-ta definiert ist), als Abgabenverpflichteter genannt, der an den Gott Poseidon (Zeile. 1), *34-ke-te-si (Zeile .2), we-da-ne-we (Zeile .3) und di-wi-jewe (Zeile. 4) Getreide (GRA) abliefert. Der $e$-qe-ta di-wi-je- $u$ ist ein Empfänger, während der e-qe-ta a-re-ku-tu-ru-wo als Lieferant auftritt. Die Funktion des einen als „Priester des Zeus“ könnte als Erklärung für die unterschiedliche Stellung der beiden $e$-qe-ta dienen, doch scheint mir noch ein weiterer Hinweis auf die herausragende Stellung des $e$-qe-ta di-wi-je-u gegenüber den sonstigen $e$-qe-ta gegeben. Dies ist die Angabe e-re-u-te-re auf PY Cn 3.1, welche sich auf PY Wa 917.2 in Kombination mit dem Wort e-qe-ta findet. Im Allgemeinen wird die Interpretation von e-re-u-te-re als „inspector“ akzeptiert ${ }^{157}$ und drückt auf Cn 3.1 vielleicht eine zusätzliche Funktion des di-wi-je-u in Bezug auf die Lieferung von Stieren aus. Wäre es nicht möglich, dass eine Person, in seiner Funktion als „Zeuspriester“ Abgaben (GRA) empfangen hätte (PY EsSerie) und derselbe Mann gleichzeitig eine hohe militärische Stellung als $e$ qe-ta innegehabt hätte, und damit verbunden ${ }^{*} e-r e-u$-te gewesen wäre ${ }^{158}$ (PY

\footnotetext{
150 Siehe S. 12.

151 Vgl. dazu Palaima 1989, 104: „It is safe to say that di-wi-je-u himself moves in high company. And so these oxen might, too“; R. Palmer 1994, 77.

${ }_{152}$ Deger-Jalkotzy 1978, 19 Fn. 42.

153 Deger-Jalkotzy 1978, 20.

154 Palaima 1989, 104.

155 Siehe S. $17 f$.

156 Palaima 1989, 104

157 L. R. Palmer 1954, 67; Ventris -Chadwick 1973, 207; Lindgren 1973 II, 51; Aura Jorro 1985, s.v. e-re-u-te-re; Palaima 1989, 104; Deger-Jalkotzy 1978, 74 u. Fn. 235, S. 75.

${ }^{158}$ Die Verbindung der beiden Titel $e$-qe-ta und e-re-u-te-re ist auch für Knossos belegt, wo auf der Tafel KN As 4493 e-pi-ko-no, e-qe-ta, e-re-u-te-[re zu lesen ist, s. dazu Melena 1975, 37ff.; Uchitel 1984, 160; Deger-Jalkotzy 1978, 74, 92ff., bes. S. 76: „Dagegen halte
} 
An 656.8.9 und Wa 917.2). Und gerade in Bezug auf die Tafel Cn 3.2 wäre es möglich anzunehmen, dass er beide Ämter in einer Person wahrnahm, und womöglich nur die Angabe der beiden höheren Titel erfolgte, also e-re-u-te-re di-wi-je-we, wobei e-re-u-te-re möglicherweise den Titel e-qe-ta inkludiert. Entsprechend den unterschiedlichen Aufgaben wäre von den Palastschreibern jeweils der zur „momentanen“ Erfüllung der Verpflichtungen geeignete Titel angegeben worden.

Wenn eine Person Verbindungen zum Kult aufweist ${ }^{159}$ und dann noch di-wije- $u$ hieß, so ist wohl kaum anzunehmen, dass diese Person im Dienste einer anderen Gottheit als der des Zeus zu suchen ist. Somit bestätigt sich die Annahme von S. DegER-JALKOTZY, sollte es sich bei di-wi-je-u um einen „Eigennamen“ handeln, dieser in Verbindung mit seiner Funktion als Angehöriger im Kult des Zeus zu verstehen ist. ${ }^{160}$ Aufgrund der Involvierung eines hohen Würdenträgers, vor allem in seiner Funktion als „Zeuspriester“ scheinen die Rinder für einen kultischen Zweck gedacht gewesen zu sein. ${ }^{161}$ J. WeILHARTNER verwies auf Xenophons Staatsverfassung der Lakedaimonier, in der berichtet

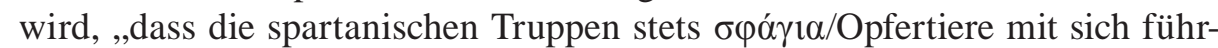
ten, um sie angesichts einer drohenden Gefahr oder wichtigen Entscheidung schlachten zu können (Xen.Lak.pol.13,3/5)“162. Des Weiteren führte er an, dass ein solches Opfer „um seiner selbst willen betrieben“ wurde und „nicht als Vorspiel zu einem kultischen Bankett" diente. ${ }^{163}$

Das einzige noch nicht eindeutig zuzuordnende Wort des Textes Cn $\mathbf{3}$ ist me-za-na (Zeile .1). Dieses wurde von L. R. PALMER als „schwarz“ in Bezug auf die Rinder in Betracht gezogen, doch ist diese Interpretation nach $\mathrm{M}$. Ventris und J. Chadwick abzulehnen und L. R. PALMER selbst erwog später in The Interpretations of Mycenaean Greek Texts den Namen einer Gottheit

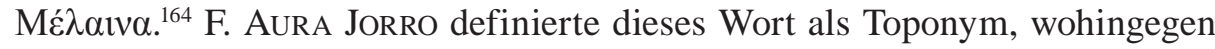
in den Documents in Mycenaean Greek me-za-na als Kollektivbezeichnung für die angeführten Mannschaften beschrieben ist, während J. T. KILLEN vorschlug me-za-na als Personennamen zu sehen. ${ }^{165}$

Die Tafel KN C 902.1-.12 nennt verschiedene Ortschaften, Ethnika, Titel und Berufsbezeichnungen. Auf jede Auflistung folgt der Eintrag von Rindern ohne Geschlechtsdefinition. Die Zeilen .1-.3 haben keine Zahlenangaben, wäh-

ich es für nicht ausgeschlossen, dass jene /heq ${ }^{\mathrm{u}}$ etai/, welche einerseits die Küstenwachen zu kontrollieren hatten, andererseits aber sicherlich auch selbst die Anmarschrouten der Feinde zur See und gegebenenfalls zu Land beobachten und darüber Bericht erstatten mussten, mit einem eigenen Titel bezeichnet wurden, nämlich /hequ etai ereutēres/. Dieser Titel wäre geeignet, die allgemeinere Bezeichnung /hequ etas/ einzuengen und für eine Spezialaufgabe näher zu definieren." Ich schließe mich dieser Meinung insoweit an, dass die e-qe-ta e$r e$-u-te-re für eine Spezialaufgabe näher definiert wurden, möchte diese jedoch mit einer hervorgehobenen Stellung gegenüber den „normalen“ e-qe-ta sehen; vgl. dazu in der PY Es-Serie den e-qe-ta di-wi-je-u als Empfänger und den e-qe-ta a-re-ku-tu-ru-wo als Lieferant.

159 In der PY Es-Serie scheint di-wi-je- $u$ als Empfänger neben dem Gott Poseidon und dem weu-da-ne-u auf; PY Cn 3 nennt ihn als „Empfänger“ von Rindern, wobei diese Tafel durch die Schreiberhand mit PY Cn 608 in Verbindung steht.

${ }^{160}$ Siehe S. 31 u. Fn. 152.

161 R. Palmer 1994, 77.

162 Weilhartner 2002, 152.

163 Weilhartner 2002, 152.

164 L. R. Palmer 1954, 67; Ventris-Chadwick 1973, 207; L. R. Palmer 1963, 175 (s. auch Weilhartner 2002, 141, 152).

165 Aura Jorro 1985, s.v. me-za-na; Ventris-Chadwick 1973, 435 (vgl. Bennet 1999, 148); Killen 1999, 330. 
rend ab Zeile .4 jeweils Bos 1 zu lesen ist. Anschließend erfolgte die Angabe von 12 Einheiten des Ideogrammes $* 170$, das mit ne genauer spezifiziert wurde.

Vier der auf KN C 902 angegebenen Ortschaften, - wa-to (Zeile .3), si-ra-ro (Zeile .4), o-du-ru-we (Zeile .6), a-pa-ta-we (Zeile .9) - sind auch in der knossischen Co-Serie vorhanden: wa-to auf Co 903.1, si-ra-ro auf Co 907.1, o-]duru-wo auf Co 910.1 und a-pa-ta-wa auf Co 909.1. Gleichzeitig enthält C 902.2 das zum Ort o-du-ro-we/-wo (C 910.a) gehörige Ethnikon o-du-ru-wi-jo.

KN

C 90

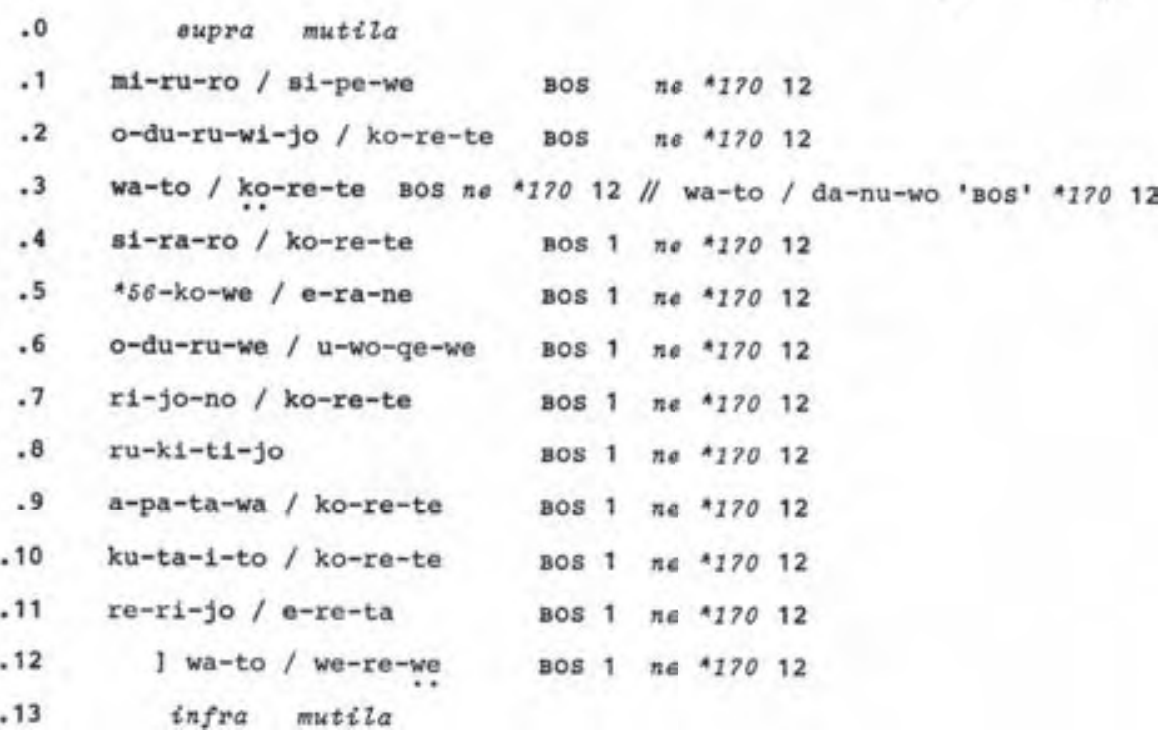

Für L. Godart ist das Auftreten derselben Ortsnamen in der KN Co-Serie und auf KN C 902 kein Zufall, sondern zeigt, dass diese Ortschaften aufgrund der gemeinsamen Auflistung auf C 902 im selben Gebiet zu lokalisieren sind, also im Westen Kretas. ${ }^{166}$ J. DrIESSEN vermutete, dass die auf C 902 genannten Orte der Verwaltung durch die ebenfalls angeführten Funktionäre anvertraut waren, „,'est-à-dire ,aux ko-re-te', aux we-re-we et aux u-wo-qe-we“ und diese Funktionen entweder von Knossiern oder lokalen Eliten wahrgenommen wurden. ${ }^{167}$

Die Interpretation des auf C 902 angeführten Ideogrammes *170 ist nicht eindeutig geklärt. In Documents in Mycenaean Greek wurde dieses Zeichen aufgrund des Zusatzes ne als junge Rinder (Kälber) oder Jungtiere einer anderen Tiergattung interpretiert. ${ }^{168}$ Dies, da das vor *170 geschriebene ne mit dem klassisch-griechischen vह́os ,jung“ verbunden wurde. ${ }^{169}$ Junge Tiere sind in den Schaftexten aus Knossos (z. B. Dh(1) 1240.A: 'ne' ki, Dk(2) 1066, Dk(2) 1067 + 5189: ' $k i$ ' ne, Dp 699.a: ne) ebenfalls mit ne determiniert, doch ist das zugehörige Ideogramm immer jenes der erwachsenen Schafe (OVIS $\left.{ }^{\mathrm{m}}\right)$. Ich denke doch, dass auch im Falle von Kälbern bei KN C 902 der Schreiber nach demselben System vorgegangen wäre und die Ideogramme Bos ${ }^{\mathrm{m}}$, Bos ${ }^{\mathrm{f}}$ oder BOS mit einem entsprechenden ne versehen hätten. Damit ist wohl auszuschließen, dass das Ideogramm *170 ein junges Rind repräsentiert.

\footnotetext{
166 Godart 1971a, 421ff.

167 Driessen 1992, 211f.; Godart 1992, 262.

168 Ventris-Chadwick 1973, 212, 437f.

169 Ventris-Chadwick 1973, 562.
} 
Möglich wäre die Interpretation als ein pflanzliches Produkt. So gibt es zwei Hinweise, dass es sich bei dem Ideogramm *170 um ein Gewürz oder eine Kräutersorte handeln könnte. Zum einen ist dieses Zeichen auf C 902 stets mit der Mengenangabe 12 (einem Vielfachen von drei) versehen und zum anderen wird es jeweils von einem einzigen Tierideogramm begleitet. Diese zwei Punkte sind zum Beispiel auch für das Ideogramm *171 charakteristisch. Dieses ist auf PY Aq 64 in den Mengen von drei, sechs und 12 Einheiten angegeben und mit EQU ZE 1 verbunden. ${ }^{170}$ Auch auf den thebanischen Tonplomben findet sich das Zeichen $* 171$ mit den Mengenangaben 30 und 36, wiederum einem Vielfachen von drei. Das Ideogramm *171 wird generell als pflanzliches Produkt (möglicherweise der Stängel des Zyperngrases) gedeutet. ${ }^{171}$ Die Vorschläge zum Gebrauch reichen dabei von Viehfutter über ein für die menschliche Ernährung gedachtes Gewürz bis zu der Verwendung in der Parfümindustrie. ${ }^{172}$ Aufgrund der Analogien (Mengeneinheiten ein Vielfaches von drei, ein Tier beziehungsweise ein Paar Tiere) könnte es sich auch bei dem Ideogramm *170 um ein pflanzliches Produkt gehandelt haben. Bereits TH. PALAIMA schlug die Interpretation als Futter vor. ${ }^{173}$ Doch lässt sich, analog zu *171, auch der Nutzen als Nahrungsmittel erklären.

Auch das zugehörige Silbenzeichen ne stellt bei der Interpretation des Ideogrammes *170 als pflanzliches Produkt kein Problem dar, wenn man von der Interpretation als véoৎ (jung) abgeht. So lautet das klassisch-griechische Wort für „frisch abgepflückt“ $v \varepsilon o \sigma \pi \alpha ́ \varsigma$ und könnte in den Linear B-Texten somit ebenfalls als abgekürztes ne geschrieben worden sein. Sollte damit ausgedrückt werden, dass hier explizit frische Kräuter, Gewürze oder sonstige pflanzliche Produkte gemeint waren, und keine getrockneten? ${ }^{174}$

L. GODART sah die Tiere und ne *170 als Lieferungen aus den verschiedensten Ortschaften an den Palast von Knossos und die mögliche Verwendung für „consommés ou sacrifiés lors de cérémonies“. ${ }^{175}$ Dass die Rinder und *170 für eine besondere Gelegenheit geliefert wurden, scheinen schon diese beiden Kommoditäten selbst zu bestätigen. Die Rinder waren mit dem bloßen Ideogramm Bos wiedergegeben, welches vermutlich unverschnittene Tiere anzeigt, die damit schon wertvoller waren als verschnittene. Sollten die Zeichen ne *170 tatsächlich für ein frisch gepflücktes pflanzliches Produkt stehen, wäre dies ein weiterer Hinweis. Bei der Interpretation der Rinder als unverschnittene Stiere und von $n e * 170$ als frische Pflanzen deutet die Aufzeichnung von $\mathbf{C}$ 902 auf die Verwendung bei einem außergewöhnlichen Ereignis, zum Beispiel einem Festmahl, hin. ${ }^{176}$

170 Einen Vergleich der Angaben von KN C 902 und PY Aq 64 stellte bereits Uchitel 1984, 159 an, wobei er anmerkte, dass Aq $\mathbf{6 4}$ zumindest zwei ko-re-te-re verzeichnete.

${ }^{171}$ R. Palmer 1999, 474 u. Fn. 43.

${ }_{172}$ R. Palmer 1999, 474ff.; Piteros-Olivier-Melena 1990, 162f.; Killen 1992, 368, 371, 375.

173 Palaima 1989, 101 u. Fn. 45.

174 Vgl. R. Palmer 1999, 476f.

175 Godart 1999, 252.

${ }^{176}$ Dr. Jörg Weilhartner wies mich in Zusammenhang mit der Tafel KN C 902 auf die Verbindung zu den Aufzeichnungen der knossischen C(3)-Serie hin (s. auch Godart 1999, 254), die hier kurz erwähnt sei. Die einzelnen Texte verzeichnen jeweils nur ein Schwein, doch auch diese wären meiner Interpretation folgend männlich und unkastriert. Und soweit zu erkennen sind in Zusammenhang mit diesen Tieren ebenfalls Funktionäre aufgeführt $-a$ to-mo, du-ma, da-mo-ko-ro. 
PY

Cn 4

.1
.2
.3
.4
.5
.6
.7
.8
.9
.10
.11

a-si-ja-ti-ja , ta-to-mo , o-pe-ro

mu-ta-pi , ku-ri-sa-to ovis $+T A \quad 22$

qe-re-me-ti-re , sa-ṇị-j̧o ovis $+T A \quad 16$

ta-to , a-ka-re-u-te, țu-ni-jo ovis $+T A 7$

e-ri-no-wo-te, ti-ri-jo ovis $+T A 7$

ne-do-wọ-te , tu-ti-je-u

ovis $+T A 4$

e-ri-to-ti-no, ne-me-ta-wo ovis $+T A 10$

wo-tu-wa-ne, e-ke-si-jo ovis $+T A 9$

ma-ta , a-ka-re-u-te , ke-ro-we ovis $+T A 7$

si-jo-wo-te, o-qe, e-ra-se ovis $+T A 10$

nacat

S4 - H21

PY

Cn 595 .1 e-ra-te-re-wa-pi , ta-to-mo , o-pe-ro ,

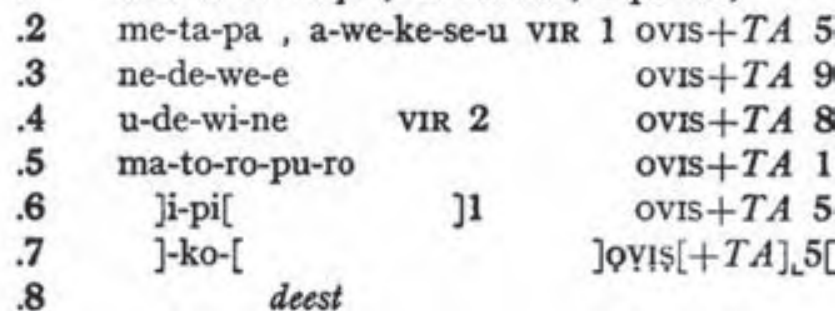

Eine in den Linear B-Texten aufgelistete Kategorie von Tieren bedarf einer zusätzlichen Besprechung - die als ovis + TA definierten Schafe der Tafeln PY Cn 4.2-.10 und Cn 595.2-.7. Die Tiere dieser beiden Texte sind als fehlend (ope-ro) verzeichnet. Sie hätten sich jedoch in den Stallungen (ta-to-mo) zweier Orte - a-si-ja-ti-ja (Cn 4.1) und e-ra-te-re-wa-pi (Cn 595.1) - befinden sollen. Für die Interpretation des Ideogrammes ovis + TA wurden zwei Möglichkeiten vorgeschlagen: 1) Mastschafe und 2) Zuchtböcke.

\subsubsection{Interpretation als Mastschafe}

P. HR. ILIEvSKI sah die als ovis + TA beschriebenen Schafe als „rams fed in steadings, both for fleece and probably meat and tallow“ “. ${ }^{177}$ Bereits M. DoRIA erwägte die Möglichkeit, dass es sich bei den Tieren von Cn 4 und Cn 595 um Schafe handelte, die wegen ihrer Wolle in einem speziellen Stall gehalten wurden. ${ }^{178}$ Die dem Ideogramm ovis zugefügte Ligatur TA wird generell als Abkürzung des ebenfalls auf den Tafeln genannten Wortes ta-to-mo ( $\sigma \tau \alpha \theta \mu o ́ s)$ gesehen. ${ }^{179}$

Wäre es tatsächlich möglich, dass der Palast eine große Anzahl von Schafen mästen ließ? Das Fett von Schafen war zwar auch in der Antike beliebt, und selbst Homer beschreibt des Öfteren fette Hammel, doch scheint die Mast von Schafen nicht in demselben Ausmaß betrieben worden zu sein wie die Schweinemast. Schafe wurden ja hauptsächlich der Wolle wegen gehalten, während das Schwein im Grunde nur als Fleisch- und Fettlieferant diente. Im Vergleich

\footnotetext{
177 Ilievski 1968, 631.

${ }^{178}$ Doria 1958, 60.

179 Lang 1966, 254; Ilievski 1968, 630; Ruijgh 1967, 32.
} 
mit dem Text PY Cn 608, der Schweine verzeichnet, die gemästet werden sollten, mutet die Angabe von fehlenden Schafen auf Cn 4 und Cn 595 doch etwas merkwürdig an. Sollte der Palast von Pylos fehlende Mastschafe verzeichnet haben? Sicher könnte man damit argumentieren, dass die Tiere, welche gemästet werden sollten, nicht geliefert wurden. Doch warum fehlen im Verhältnis zu den vorhandenen zu mästenden Schweinen von Cn 608 (25 Stück) so viele Schafe auf Cn 4 (92 Stück) und Cn 595 (33 Stück)? Des Weiteren ist zu beachten, dass in den Linear B-Texten die Masttiere mit dem jeweiligen Ideogramm

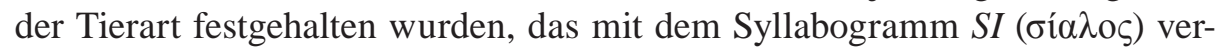
sehen war. ${ }^{180}$ So finden sich Mastschweine (sUS $+S I$ ) auf den Tafeln PY Cn 608.3.4.5.6.7.8.9.10.11, PY Ua 25.1, Un 2.6, Un 138.4 und auf den Tonplomben TH Wu 52. $\alpha$ und Wu 68. $\alpha$. Mastrinder (BOS + SI) dagegen sind in dem Text von PY Cn 418.2.3 verzeichnet. Wenn sowohl die Ideogramme der Mastschweine als auch jene der Mastrinder mit dem Syllabogramm SI versehen wurden, ist es gerechtfertigt anzunehmen, dass auch etwaige Mastschafe als *OVIS + SI bezeichnet worden wären. Somit dürfte das mit der Ligatur TA versehene Ideogramm ovIs (= ovIS $+T A$ ) nicht als Beschreibung von Mastschafen gesehen werden.

\subsubsection{Interpretation als Zuchtböcke}

M. LANG interpretierte die als OvIS + TA erfassten Schafe als Zuchtböcke. ${ }^{181}$ Die Widder der klassischen Zeit sollten vor der Paarung von der Herde abgesondert, kräftig gefüttert und im Stall noch mit Gerste gestärkt werden. ${ }^{182}$ Auch M. LANG setzte das dem Ideogramm ovis zugefügte Syllabogramm TA mit dem Wort ta-to-mo gleich. ${ }^{183}$ Nachdem sich die den Ideogrammen zugefügten Silbenzeichen jedoch jeweils auf die körperlichen Eigenschaften der Tiere beziehen (vgl. sUS $+S I$, sUS $+K A$, ta BOS, BOS $+S I$ ), ist zu überlegen, ob dies nicht auch im Falle von ovIs + TA zutrifft. Wenn es sich bei diesen Schafen um Zuchtböcke gehandelt hatte, sollte die Ligatur TA dieses auch zum Ausdruck gebracht haben. Doch die klassisch-griechischen Wörter für Widder sind

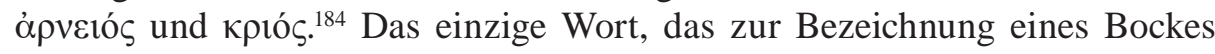
verwendet wurde und im Mykenischen als TA geschrieben hätte werden können, war $\tau \rho \alpha ́ \gamma o \varsigma$, womit allerdings der Ziegenbock gemeint war. Wäre es nicht möglich, dass zur mykenischen Zeit ein Zuchtbock - egal ob Ziege oder Schaf - als $\tau$ ‘́́ $\gamma o \varsigma$ bezeichnet wurde? ${ }^{185}$ Zumindest scheint in klassischer Zeit die Trennung zwischen Schafen und Ziegen in ihrer Bedeutung als Kleinvieh nicht sehr stark gewesen zu sein. „Eine Besonderheit des Griechischen ist es, dass das gezähmte Tier [Anm. Ziege] in der Herde durch die Kollektivbezeichnungen $\tau \grave{\alpha} \mu \tilde{\eta} \lambda \alpha$ und $\tau \grave{\alpha} \pi \rho o ́ \beta \alpha \tau \alpha$ derart eng mit dem Schaf verbunden ist, dass eine Unterscheidung in der Regel ausgeschlossen ist“ ${ }^{186}$ Auch das Mykenische scheint, bis auf die Wollproduktion, keinen besonderen Unterschied von Schaf und Ziege gekannt zu haben. Dies zeigt sich deutlich in der pylischen Ae-Serie, wo auf

\footnotetext{
180 Siehe S. 7, 17.

${ }^{181}$ Lang 1966, 254; s. auch Halstead 1990-91, 358.

182 Magerstedt 1859, 116; Orth 1921, 385; Varro rust.2,2,13.

183 Lang 1966, 254.

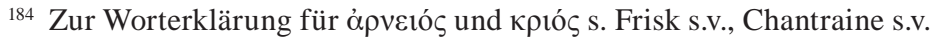

185 Auch das Deutsche kennt den Ausdruck „Bock“ in Verwendung als Ziegenbock und Schafsbock, wobei für letzteres gleichzeitig das Wort „Widder“ gegeben ist.

${ }^{186}$ Richter 1972, 399.
} 
PY Ae 108 ein $a_{3}$-ki-pa-ta (,Ziegenhirte“) in Verbindung mit qe-to-ro-po-pi („Vierfüßler“) genannt wird, während auf PY Ae 134 ein po-me („Hirte“) mit qe-to-ro-po-pi zu tun hatte. Aus diesem Grunde würde ich es nicht absolut ausschließen, dass in mykenischer Zeit auch ein Schafsbock als *tragos (OVIS + TA) bezeichnet werden konnte.

\subsubsection{Das Ideogramm CAP $+E$}

PY

Cn $418+$ Xn $1471+f r$.

(Cn; Ciii)

187

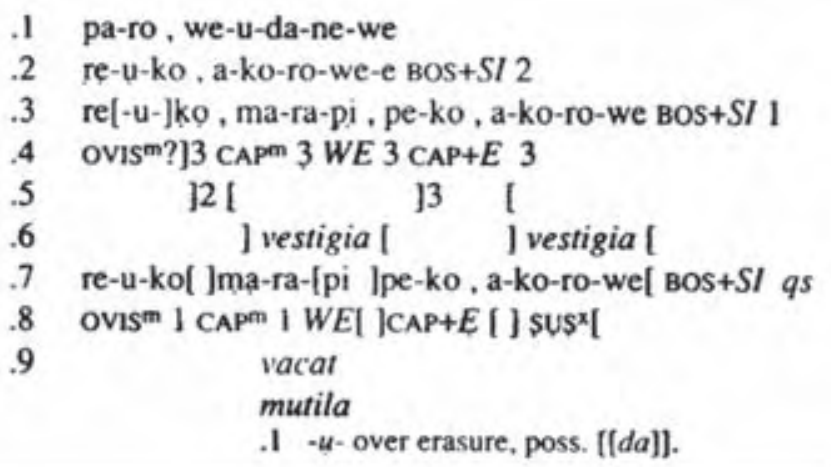

Auf der Tafel PY Cn 418.4.8 werden Ziegen genannte, die mit dem Ideogramm $\mathrm{CAP}+E$ wiedergegeben sind. J. L. MelenA interpretierte die Angabe CAP $+E$ als

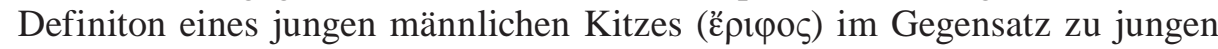
Schafen, welche als WE (*wetalon) definiert würden. ${ }^{188}$ Doch zeigt gerade eine Tonplombe aus Theben, TH Wu 74, dass auch eine Ziege mit dem Silbenzeichen we als Jungtier (we CAP ${ }^{\mathrm{m}}$ ) definiert wurde. Und die Tafel PY Cn 418 weist in den Zeilen .4 und .8 selbst das Zeichen WE auf, wobei es jeweils auf die Angabe einer unterschiedlichen Anzahl von männlichen Ziegen folgt.

Aufgrund der Interpretation der Ideogramme sus $+S I$, sus $+K A$, BOS $+S I$, ta BOS und ovIS $+T A$ sollte auch das Syllabogramm $E$ dahingehend gedeutet werden, dass es sich auf eine körperliche Eigenschaft der Tiere bezieht. Im klassischen Griechisch findet sich ein Wort, das sich hervorragend eignet, das

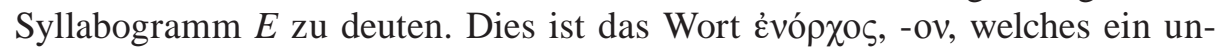
kastriertes Tier (nicht jedoch ein Zuchttier) beschreibt. Damit lässt sich hinter dem Ideogramm CAP $+E$ eine unkastrierte männliche Ziege vermuten. Im Falle von Cn 418 dürfen wir demnach vielleicht von einem als unkastriert bezeichnetem Tier, das für den Kult vorgesehen war, ausgehen. Auch in der Ilias dient

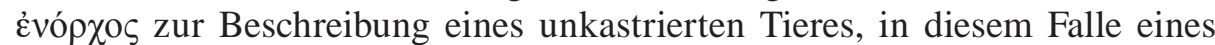

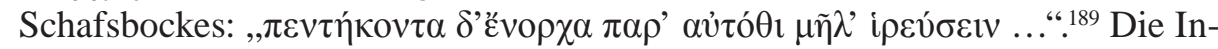
terpretation der Ziegenböcke von Cn 418.4.8 als als Opfertiere wird durch die Angabe der Rinder (Cn 418.2.3), welche wohl als Kultopfer zu sehen sind zusätzlich unterstützt. ${ }^{190}$ Sollte das Ideogramm CAP $+E$ ausdrücklich verdeutlichen, dass es sich bei den Ziegen - im Gegensatz zu den vermutlich kastrierten Mastrindern (BOS +SI) - um nicht kastrierte Tiere handelte?

\footnotetext{
187 Join nach Melena 1994-1995, 95f.

188 Melena 1994-95, 96.

189 Hom.Il.23,147.

190 Siehe S. 17ff.
} 
Die Schlussfolgerung der vorangegangenen Interpretationen lautet, dass die einfachen Ideogramme BOS, sUS, OVIS, CAP, EQU, CERV jeweils den männlichen unkastrierten Vertreter der jeweiligen Gattungen zuzuschreiben sind. Diese Zeichen dienten als Ausgangspunkt für die Einordnung der zu den jeweiligen Tieren gehörenden weiblichen Pendants, deren Ideogramme Bos ${ }^{\mathrm{f}}$, sus ${ }^{\mathrm{f}}$, ovis $\mathrm{f}$, $\mathrm{CAP}^{\mathrm{f}}$, EQU ${ }^{\mathrm{f}}$ mit einem zusätzlichen vertikalen Strich versehen wurden, und der männlichen kastrierten Tiere, deren Ideogramme BOS ${ }^{\mathrm{m}}, \mathrm{SuS}^{\mathrm{m}}$, ovIS ${ }^{\mathrm{m}}, \mathrm{CAP}^{\mathrm{m}}, o-n o$ $\mathrm{EQU}^{\mathrm{m}}$ mit zwei horizontalen Strichen gekennzeichnet sind. Des Weiteren folgte die Angabe von Mastrindern und Mastschweinen durch den Zusatz des Silbenzeichens SI (BOS + SI, SUS + SI) und von speziellen Zuchttieren beziehungsweise explizit nicht kastrierten männlichen Tieren durch das Zufügen von $K A$, $T A$, $t a$ und $E$ (sus $+K A$, ovIs $+T A$, $t a$ BOS, CAP $+E$ ) - der jeweils verkürzten Form der

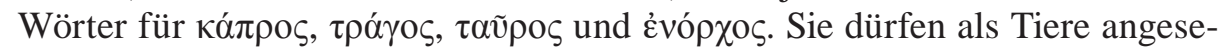
hen werden, die im besten Alter für die Zucht standen (sus + KA, ovIs + TA, ta BOS) beziehungsweise für den Gebrauch als Opfertiere im Kult ausdrücklich als nicht kastriert $(\mathrm{CAP}+E)$ bezeichnet wurden. Bereits 1992 merkte TH. PALAimA an, dass das dem Ideogramm Bos auf KN C(1) 901 zugefügte ta auf einen Unterschied dieses Rindes zu den sonstigen in den Linear B-Texten verzeichneten Rindern hinweisen muss; er ging jedoch noch davon aus, dass Bos $^{\mathrm{m}}$ allgemein die männlichen Tiere charakterisiere: „But then the use of the unsexed ideogram is very odd, unless Scribe 107 viewed the sex-marks as redundant after stipulating that this animal was a bull. The use of this special adjunct reference at least should imply a difference between the $t a$ animal and the normal male oxen listed on the texts...”. ${ }^{191}$

Die zusätzliche Kennzeichnung als sus $+K A$, ovIs $+T A$, und ta BOs zu den einfachen Ideogrammen sUs, ovIS und BOS ist verständlich, wenn man bedenkt, dass die männlichen Tiere nur für einen bestimmten Zeitraum für die Zucht geeignet sind. Die Zeichen sollen wohl nicht nur die Unversehrtheit der Tiere in Bezug auf ihre Fortpflanzungsfähigkeit angeben, sondern auch noch auf eine bestimmte Lebensphase des Tieres hindeuten. Dies wird besonders bei den als ovis + TA definierten Schafen der Tafeln PY Cn 4 und Cn 595 deutlich, die sich in Zuchtstallungen hätten befinden sollen. Das Zuchtvieh durfte dabei weder zu jung noch zu alt für die Zeugung sein, und es wurde eine Auswahl der geeigneten Tiere getroffen. Die antike Literatur liefert uns detaillierte Angaben über das optimale Zuchtalter der männlichen und weiblichen Haustiere. ${ }^{192}$ Nach Columella sollten Stiere ein Alter von vier bis 12 Jahren aufweisen: „Von den Bullen werden diejenigen von der Zucht ausgeschlossen, die weniger als vier und mehr als zwölf Jahre alt sind; die einen, weil sie wegen ihrer Jugend zur Zeugung von Arbeitstieren als noch ungeeignet gelten, die andern, weil sie durch das Alter entkräftet sind. “'193 Desgleichen sollten sie „möglichst großgliedrige, gutartige und in mittlerem Alter stehende Zuchtstiere ${ }^{\text {‘194 }}$ sein. Eber hingegen galten von einem bis vier Jahren als geeignet: „Im Alter von ein bis vier Jahren zeugen sie am besten, ... ${ }^{“ 195}$ und sollten folgende Eigenschaften

\footnotetext{
191 Palaima 1992, 470.

192 Für eine Zusammenfassung der Angaben antiker Autoren, wie z. B. Columella, Varro und Plinius, zu den verschiedensten Tierarten s. Benecke 1994, 182ff. und Peters 1998, 28f., 80f., $112 f$.

193 Colum.6,24,1.

${ }^{194}$ Colum.6,20.

195 Colum.7,9,2.
} 
aufweisen: „,... Eber von großer Mächtigkeit des Gesamtkörpers, doch mehr von vierkantiger als langgestreckter oder rundlicher Gestalt, mit hervortretendem Bauch, großen Hinterbacken und folglich nicht sehr hohen Beinen und Zehen, mit kräftigem und muskulösem Nacken und kurzem, zurückgestülptem Rüssel. ${ }^{“ 196}$ Bei den Schafen galten diejenigen als für die Zucht geeignet, deren „Gestalt hochgewachsen und stattlich ist, der Bauch hervortretend und wollig, der Schwanz sehr lang und dicht behaart, die Stirn breit, die Hoden groß, die Hörner gewunden, ... ${ }^{\text {“197 }}$ und sie sollten zwischen drei und acht Jahren alt sein. ${ }^{198}$

Die folgende Tabelle (Tab. 5) soll die unterschiedlichen Tierideogramme der Linear B-Texte und ihre Bedeutung in Bezug auf die einzelnen Tiere wiedergeben.

\begin{tabular}{|c|c|c|c|c|c|c|c|}
\hline & $\begin{array}{l}\text { unkastrierte } \\
\text { männliche } \\
\text { Tiere }\end{array}$ & $\begin{array}{l}\text { weibliche } \\
\text { Tiere }\end{array}$ & $\begin{array}{l}\text { kastrierte } \\
\text { männliche } \\
\text { Tiere }\end{array}$ & $\begin{array}{l}\text { Masttiere } \\
\text { (wohl } \\
\text { männlich) }\end{array}$ & $\begin{array}{l}\text { unkastrierte } \\
\text { männliche } \\
\text { Zucht-Tiere }\end{array}$ & Jungtiere & $\begin{array}{l}\text { männliche/ } \\
\text { weibliche } \\
\text { alte Tiere }\end{array}$ \\
\hline $\begin{array}{l}* 109 \\
\text { Rind }\end{array}$ & BOS & $\mathrm{BOS}^{\mathrm{f}}$ & $\operatorname{BOS}^{\mathrm{m}}$ & $\mathrm{BOS}+S I$ & ta BOS & ne we BOs $^{\mathrm{m}}$ & \\
\hline $\begin{array}{l}* 108 \\
\text { Schwein }\end{array}$ & SUS & SUS $^{\mathrm{f}}$ & SUS $^{\mathrm{m}}$ & $\mathrm{SUS}+S I$ & $\mathrm{SUS}+K A$ & & \\
\hline $\begin{array}{l}\text { *106 } \\
\text { Schaf }\end{array}$ & OVIS & OVIS $^{f}$ & OVIS $^{\mathrm{m}}$ & & OVIS + TA & 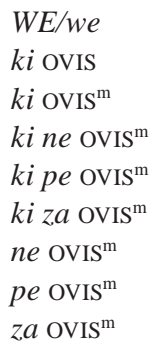 & $\begin{array}{l}\text { pa ovis }{ }^{\mathrm{m}} \\
\text { pa-ra-jo } \\
\text { OVIS }^{\mathrm{m}}\end{array}$ \\
\hline $\begin{array}{l}\text { *107 } \\
\text { Ziege }\end{array}$ & $\begin{array}{l}\text { CAP } \\
\mathrm{CAP}+E\end{array}$ & $\mathrm{CAP}^{\mathrm{f}}$ & $\mathrm{CAP}^{\mathrm{m}}$ & & & $\begin{array}{l}\text { WE/we } \\
\text { ki CAP }\end{array}$ & \\
\hline $\begin{array}{l}* 105 \\
i-q 0 \\
\text { Pferd }\end{array}$ & EQU & $E^{f} U^{f}$ & & & & po-ro EQU & \\
\hline $\begin{array}{l}* 105 \\
\text { o-no } \\
\text { Esel }\end{array}$ & & $\mathrm{EQU}^{\mathrm{f}}$ & $\mathrm{EQU}^{\mathrm{m}}$ & & & po-ro EQU & \\
\hline $\begin{array}{l}* 104 \\
\text { Hirsch }\end{array}$ & CERV & & & & & & \\
\hline
\end{tabular}

Tab. 5: Mögliche Interpretation der verschiedenen Tierideogramme.

\section{Die mit der Tierhaltung verbundenen Personen}

In diesem Kapitel sollen diejenigen Personen besprochen werden, deren Namen in Texten verzeichnet sind, die sich mit der Tierhaltung von Pylos und Knossos beschäftigen. Als Ausgangspunkte dienen dabei die Angaben der knossischen D-Serien und der pylischen Cn-Serie. Es stellt sich die Frage, welche Stellung die einzelnen Personen innehatten und welche Aussagen über

${ }^{196}$ Colum.7,9,1.

197 Colum.7,3,3.

198 Colum.7,3,6. 
deren gesellschaftliche Stellung (gesellschaftlicher Rang, Beruf, Aufgabengebiete im Rahmen der Tierhaltung) durch die Textinhalte vorgegeben sind.

\subsection{Die PYlisChe CN-SeRIE, DIE „COLLECTORS“ UND „HirTEN“ SOWIE DER Vergleich Zu den AufZeichnungen der KN D-, DA-Dv-Serien}

In diesem Abschnitt soll auf die Tafeln der pylischen Cn-Serie und ihre Aussagen eingegangen werden, welche, wie sich zeigen wird, durchaus keinem einheitlichen Schema entsprechen, sondern vielmehr Teilbereiche der im Reich von Pylos durchgeführten Administration der Tierhaltung widerspiegeln. ${ }^{199}$ Des Weiteren ist zu erkennen, dass die knossischen Texte der D-, Da-Dv-Serien markante Unterschiede zum Inhalt der pylischen Cn-Serie aufweisen, die aber dazu beitragen, tiefgreifende Erkenntnisse zur Organisation und Verwaltung der Tierhaltung durch die mykenischen Paläste zu erlangen.

\subsubsection{Tiere mit den zusätzlichen Angaben pa-ra-jo, wo-ne-we, „Collectors“-} Namen und solche ohne nähere Spezifikation

Die Texte enthalten Ortsnamen, „Hirten“-Namen und die Angabe von Schafen, Ziegen und Schweinen, wobei für jede einzelne Eintragung von Tierherden ein Toponym angegeben wurde. Es erfolgte also keine zusammenfassende Aufzählung der Tiere für bestimmte Gemeinden. Daneben verzeichnen die Texte entweder 1) das Wort pa-ra-jo beziehungsweise 2) den Begriff wo-ne-we oder 3) einen „Collector“-Namen (mit oder ohne dem Terminus a-ko-ra); es finden sich aber auch Zeilen und Tafeln, die 4) ausschließlich Ortsnamen und „Hirten“-Namen anführen ohne weiteren Zusatz. Wie sich herausstellen wird, lohnt es sich durchaus, die verschiedenen Varianten der Angaben im Einzelnen durchzubesprechen.

\subsubsection{1. pa-ra-jo ovis ${ }^{\mathrm{m}}$}

PY

Cn 40

wa-no-jo , wo-wo , pa-ro , ne-ti-ja-no-re , pa-ra-jo

$\mathrm{S} 4-\mathrm{H} 21$

.2

wa-no-jo , wo-wo , pa-ro , po-so-pe-re-i , wo-ne-we

ovis $^{\mathrm{m}} 140$

.3

wa-no-jo , wo-wo , pa-ro , zo-wi-jo , a-ko-so-ta-o

ovis $^{\text {m }} 75$

.4

wa-no-jo , wo-wo , pạ-rio , po-ru-qo-ta , we-da-ne-wo

ovis $^{\mathrm{m}} 70$

.5

e-ko-me-no , pa-ro , pa-ta , pa-ra-jo

ovis $^{\mathrm{m}} 60$

e-ko-me-no , pa-ro , [ ]ma-te-we, we-da-ne-wo

ovis $^{\mathrm{m}} 80$

.6

a-ne-u-te , pa-ro , ma-ri-ti-wi-jo , a-ko-so-ta-o

ovis $^{\text {in }} 70$

.7

ma-ro-pi , pa-ro , ro-ko, pa-ra-jo

ovis $^{\text {th }} 83$

.8

ma-ro-pi , pa-ro, ka-da-ro, we-da-ne-wo

ovis $^{\text {m }} 150$

9

ma-ro , pa-ro , tu-ri-ta , a-ke-o-jo

ovis $^{\mathrm{m}} 85$

re-pe-u-ri-jo , pa-ro , e-zo-wo , a[-ko-so-]ța-o

ovis $^{\text {th }} 80$

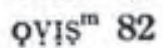

ma-ro , pa-ro , ma-u-ti-jo , a-ko-şo-ta-o

ovis' 60

a-ne-u-te pa-ro , ka-ta-wa , a-ko-so-ta

ovrs' 80

.13

a-te-re-wi-ja , pa-ro , e-wi-te-we , a-ke-o-jo

ovis $^{4} 70$

$.15-16$

vacant

${ }^{199}$ Eine Zusammenfassung unterschiedlicher Interpretationen zur pylischen Cn-Serie bietet Killen 1993, $212 \mathrm{ff.}$ 
PY

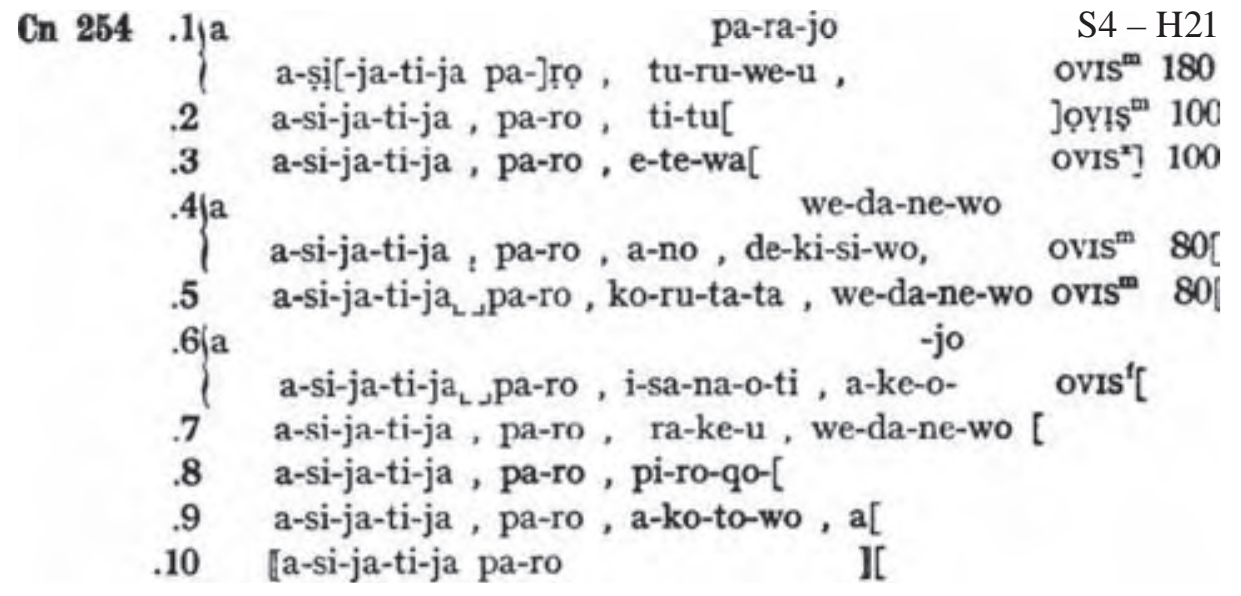

PY

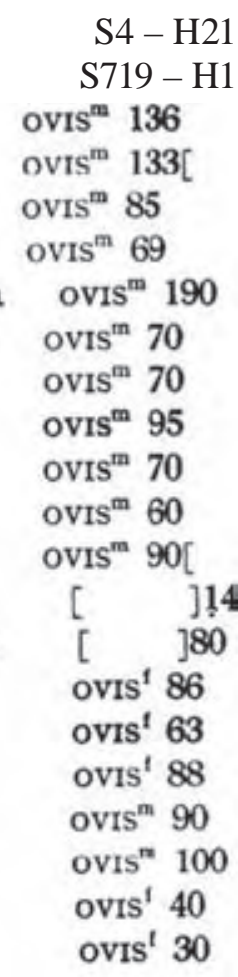

PY

\begin{tabular}{|c|c|c|c|}
\hline \multirow[t]{12}{*}{ Cn 719} & .1 & ma-ro-pi , ka-do-wo, a-ke-o-jo & ovIs $^{\text {m }} 40 \quad \mathrm{~S} 719-\mathrm{H} 1$ \\
\hline & .2 & ma-ro-pi , to-si-ta, a-ke-o-jo & ovis $^{m} 82$ \\
\hline & .3 & ma-ro-pi, me-ta-no, a-ke-o-jo & ovIs $^{f} 60$ \\
\hline & .4 & pi-*82, ma-ra-ni-jo , pa-ra-jo & ovis $^{\mathrm{ma}} 230$ \\
\hline & .5 & pi- $* 82,0-k u-k a$, a-ke-o-jo & ovis $^{\mathrm{m}} 70$ \\
\hline & 6 & pi-] $* 82$, ra-mi-ni-jo, a-ke-o-jo & ovis $^{m} 90$ \\
\hline & .7 & pi-*82 ] ku-pi-ri-jo , a-ke-o-jo & ovis $^{\mathrm{ni}} 60$ \\
\hline & .8 & pi-*82 ku-]ka-ra-so, a-so-ta-o & ovis't $] 30$ \\
\hline & .9 & wi-]ja-we-ra ${ }_{2}$, ko-ru-no, pa-ra-jo & QYฺा \\
\hline & .10 & a-pa-]re-u-pi , pa-pa-ro, a-ko-so-ta-c & ovis $^{\mathrm{a}} 100$ \\
\hline & .11 & wi-ja-we-ra ${ }_{2}$, a-ka-ma-wo , a-ko[-so- & ta-o ovis ${ }^{m} 96$ \\
\hline & .12 & wi-ja-we-ra ${ }_{2}$, a-ke-ta , wọ[-ne-]we & ovIs $^{\mathrm{m}} 100$ \\
\hline
\end{tabular}


Das Wort pa-ra-jo wird mit dem klassisch-griechischen $\pi \alpha \lambda \alpha$ ı́ $\varsigma$ (,alt“) verbunden. ${ }^{200} \mathrm{~J}$. T. KILLEN deutete die Angabe von pa-ra-jo in Zusammenhang mit Schafen dahingehend, dass diese mit fünf bis sechs Jahren für die Wollproduktion zu alt wurden und somit aus dem Herdenbestand ausgesondert werden sollten. ${ }^{201}$ L. GODART sah in der Angabe von pa-ra-jo ovIS ${ }^{\mathrm{m}}$ alte Schafe, die durch neue (jüngere) Schafe ersetzt wurden. ${ }^{202}$ Dazu setzte er am Beispiel des Ortes ma-ro-pi die als pa-ra-jo bezeichneten Schafe der Texte Cn 40.8 und Cn 655.1.2.7.8.9.10 mit den männlichen Schafen von Cn 40.7.9.10, Cn 655.3.4.17.18 und $\mathbf{C n}$ 719.1.2 gleich (s. Tab. 6).

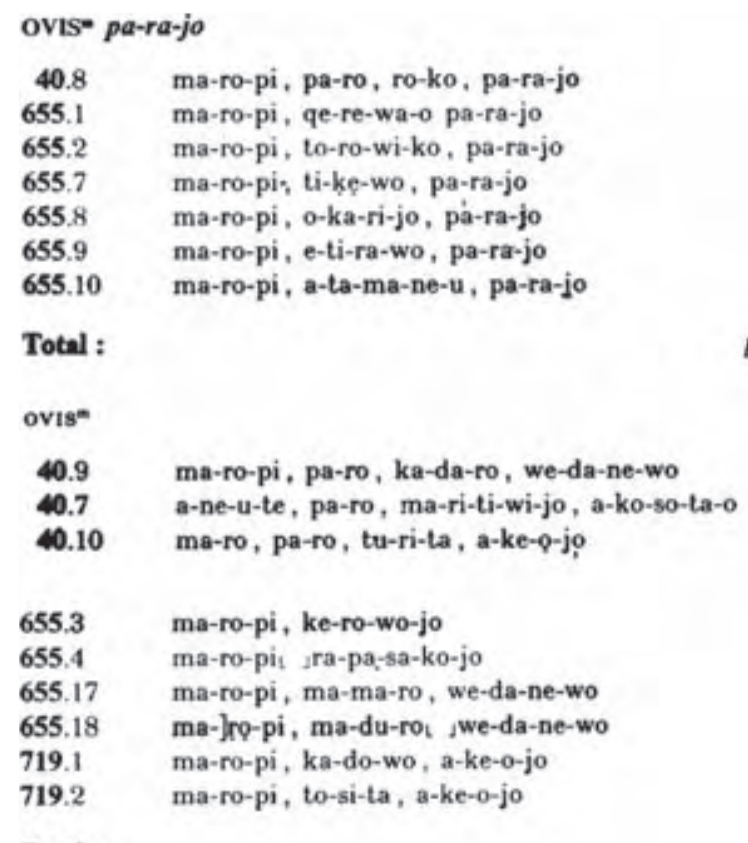

Total :

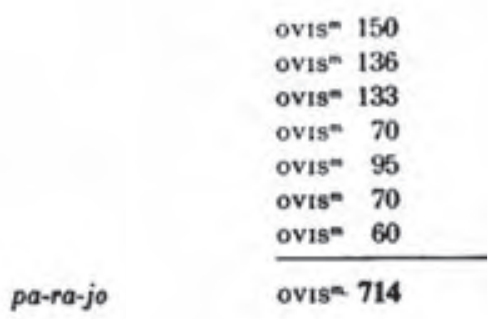

ovis= 85

ovis" 83

ovis" 80

ovis - 85

ovis" 69

ovis" 90

ovis= 100

ovis - 40

ovis" 82

ovis- 714

Tab. 6: Verhältnis von ovis ${ }^{\mathrm{m}}$ zu pa-ra-jo ovis ${ }^{\mathrm{m}}$ (nach Godart 1992, 259f.).

Ich schließe mich der Interpretation J. T. KILLENS und L. GoDARTS an, wonach die Angabe pa-ra-jo ovIS $^{\mathrm{m}}$ alte Schafe qualifiziert, die vom Palast als nicht mehr benötigt angesehen wurden und daher ausgegliedert werden sollten. Doch lassen sich die Aufzeichnungen über alte Schafe für den Ort ma-ro-pi nicht mit jenen männlichen Schafen vergleichen, die von L. GODART angeführt wurden. So dürfen die Schafe, welche für $a$-ne-u-te verzeichnet wurden, nicht zu den für ma-ro-pi angesetzten Tieren hinzugerechnet werden. ${ }^{203}$ Den Schreibern der Tafeln Cn 40, Cn 655 und Cn 719 lag offensichtlich viel an der genauen Erfassung der jeweiligen Ortschaften, da diese in jeder einzelnen Zeile angeführt wurden. L. GODART fasste für seine Berechnung alle männlichen Schafe der drei Texte zusammen, ließ jedoch jene, die mit dem Terminus $a$ ko-ra verbunden sind (Cn 655.5.6.11.12.13) außer Acht, da diese „servent sans dout à rendre compte d'une autre réalité" und deshalb nicht mit pa-ra-jo ovis ${ }^{\mathrm{m}}$

200 Bartoněk 2003, 303; Godart 1971b, 90.

201 Killen 1964, 13; Halstead 1990-91, 345; Ilievski 1968, 623.

202 Godart 1992, 259ff.

203 Godart 1992, 259 begründet die Hinzurechnung von a-ne-u-te zu ma-ro-pi mit einer Verbindung der beiden Orte zueinander. Doch könnte dies genauso gut für die weiteren Orte von PY Cn 40 und Cn 719 angenommen werden, da in den einzelnen Texten der Cn-Serie wohl die Gemeinden, die sich im selben geographischen Gebiet befanden, auf einer Tafel gemeinsam erfasst wurden. Dies scheint für Cn 40, Cn 719 und Cn 655 im nördlichen Einzugsbereich, um pi-*82 in der Diesseitigen Provinz, gelegen zu haben. 
gleichgesetzt werden könnten. Die Schafe, welche mit dem Terminus a-ko-ra verzeichnet sind, gehören wahrscheinlich tatsächlich einem anderen „Vergabemodus“ an. Dieser kann jedoch nicht so verschieden von der Vergabe der sonstigen männlichen Tiere gewesen sein, da alle Schafe immerhin in denselben Aufzeichnungen registriert wurden. Das als $a$-ko-ra OvIS $^{\mathrm{m}}$ bezeichnete Vieh sollte also nicht aus der Berechnung herausgenommen werden. ${ }^{204}$ So scheint es ein rein willkürliches Weglassen zu sein, da zum Beispiel auch die Schafe von Cn 655.3.4 anscheinend „anderen Bedingungen“ unterworfen waren, wenn man bedenkt, dass in diesen beiden Zeilen kein „Collector“-Name zu finden ist, sondern nur „Hirten“-Namen vorhanden sind. Des Weiteren konnte J. T. KILLEn für die knossische Da-Dg-Serie nachweisen, dass die als pa-ra-jo OvIS $^{\mathrm{m}}$ deklarierten Tiere sowohl männliche als auch weibliche Schafe waren. ${ }^{205}$ Wenn die pa-ra-jo-Schafe der pylischen $\mathbf{C n}$-Serie als alte, auszugliederne Tiere zu sehen sind, darf angenommen werden, dass in diesen Texten unter dem Ideogramm ovIs ${ }^{\mathrm{m}}$ ebenfalls männliche und weibliche wollproduzierende Schafe erfasst wurden. ${ }^{206}$ Dies heißt, dass bei einem Vergleich der Anzahl der mit pa-ra-jo ovis ${ }^{\mathrm{m}}$ angegebenen Tiere mit den anderen Schafen der Tafeln Cn 40, Cn 655 und Cn 719 neben den männlichen (OVIS ${ }^{\mathrm{m}}$ ) auch die weiblichen Tiere (ovIs ${ }^{f}$ ) von Cn 40.12, Cn 655.14.15.16.19.20 und $\mathbf{C n}$ 719.3 herangezogen wer-

${ }^{204}$ Godart 1992, 262 nennt drei Punkte, die belegen sollen, dass die mit $a$-ko-ra verbunden Schafe nicht mit den sonstigen Tieren der pylischen $\mathbf{C n}$-Serie vergleichbar sind:

„1) le mot $a$-ko-ra a un rôle bien spécifique au sein des tablettes de la série $\mathbf{C n}$ puisque les troupeaux associés à ce vocable se distinguent de tous les autres troupeaux d'ovins attestés à ma-ro-pi“. Die Tiere können sich jedoch nicht soviel von von den anderen Schafen unterscheiden, da ihre Aufzeichnung in denselben Texten erfolgte. Wenn der Unterschied so groß war, warum wurde dann keine eigene Tafel für alle $a$-ko-ra-Tiere angefertigt?

„2) il est exclu que le terme $a$-ko-ra puisse être sous-entendu à la suite du nom au génitif qui accompagne les enregistrements d'ovins où figurent tant l'indication du berger que celle de la localité où ce dernier exerce son activité“. Dieser Aussage kann ich zustimmen, was jedoch nicht heißt, dass damit eine Begründung gegeben ist, die mit $a$-ko-ra verbundenen Schafe nicht wie die anderen Tiere zu sehen. Der Begriff $a-k o{ }^{\circ}{ }^{\circ}$ bezieht sich auf eine Vergabe von Tieren an Personen, definiert damit den „Vergabemodus“. Dies macht jedoch keinen Unterschied zu den sonstigen Schafen, Ziegen und Schweinen der Cn-Serie, wenn wir davon ausgehen, dass alle Tiere, welche mit einem „Collector“-Namen verbunden sind, auch an diese vergeben wurden; s. dazu S. 49ff.

„3) il est donc clair que les génitifs qui affectent, la plupart du temps à Pylos et souvent à Knossos, les anthroponymes intervenant dans les enregistrements de petit bétail à la suite du nom du berger, ne sont en aucun cas des compléments déterminatifs du mot a-ko-ra puisque ce terme a une valeur spécifique et ne peut jamais etre sous-entendu; ces personnages ne sont donc pas des «Collecteurs» et ce terme entré par la grande porte dans la terminologie mycénologique est très certainement impropre pour rendre compte du rôle et de l'activité de ces individus“. Auch dieser Aussage ist zuzustimmen, doch sind gerade in Pylos die vier „Collectors“ we-u-da-ne-u, a-pi-me-de, a-ke-o und a-ko-so-ta eben auch dieselben Personen, deren Namen im Genitiv mit und ohne $a-k o-r a$ verzeichnet sind. Damit können die Tiere ohne $a$-ko-ra-Definition durchaus weiterhin als an die „Collectors“ vergeben angesehen werden, während jene mit $a$-ko-ra vielleicht einem speziellen Vergabemodus unterliegen.

205 Killen 1964, 13 Fn. 65; Halstead 1990-91, 354 Fn. 31 merkte jedoch an, dass in Pylos nur männliche Tiere als pa-ra-jo ovIs ${ }^{\mathrm{m}}$ erfasst wurden. P. Halstead ging, wie L. Godart, jedoch davon aus, dass die als pa-ra-jo ovis ${ }^{\mathrm{m}}$ deklarierten Schafe von ma-ro(-pi) und pi-*82 die gleiche Anzahl aufweisen wie die männlichen Schafe derselben Orte ohne den Definitionen pa-ra-jo, wo-ne-we, we-re-ke und a-ko-ra (s. dazu Halstead 1990-91, 355f u. Fn. 33). Wie ich in diesem Kapitel ausführe, kann diese Gleichsetzung von ovIS ${ }^{\mathrm{m}}$ zu pa-ra-jo ovIS ${ }^{\mathrm{m}}$ jedoch nicht durchgeführt werden.

${ }^{206}$ John T. Killen bestätigt in der schriftlichen Mitteilung vom 1. August 2005, dass in den Textaufzeichnungen auch weibliche Tiere zu erwarten sind. 
den sollten, da diese ja ebenfalls ausgetauscht würden. Doch damit wäre die Interpretation der pylischen Cn-Serie als Aufzeichnung über den Austausch von alten Schafen durch jüngere hinfällig, da die Gesamtzahlen der Tiere, wie die folgende Tabelle (Tab. 7) veranschaulicht, nicht mehr kongruieren würde.

Der einzige Grund der Aufzeichnung von alten (männlichen und weiblichen) Schafen in der pylischen $\mathbf{C n}$-Serie dürfte darin bestanden haben, dass die Palastverwaltung erfuhr, wieviele Tiere nicht mehr für die Wollproduktion zur Verfügung standen, da sie aus den palatialen Herden ausgegliedert wurden.

\begin{tabular}{|c|c|c|c|c|c|c|c|}
\hline & OVIS $^{\mathrm{m}}$ & & OVIS $^{\mathrm{f}}$ & $\begin{array}{l}\text { Gesamtzahl } \\
\text { ovIS }^{m}+\text { ovIS }^{f}\end{array}$ & & & pa-ra-jo ovIs ${ }^{\mathrm{m}}$ \\
\hline Cn 40.9 & 85 & Cn 40.12 & 60 & & & Cn 40.8 & 150 \\
\hline Cn 40.10 & 80 & Cn 655.14 & 86 & & & Cn 655.1 & 136 \\
\hline Cn 655.3 & 85 & Cn 655.15 & 63 & & & Cn 655.2 & 133 \\
\hline Cn 655.4 & 69 & Cn 655.16 & 88 & & & Cn 655.7 & 70 \\
\hline Cn 655.5 & 190 & Cn 655.19 & 40 & & & Cn 655.8 & 95 \\
\hline Cn 655.6 & 70 & Cn 655.20 & 30 & & & Cn 655.9 & 70 \\
\hline Cn 655.11 & 90[ & Cn 719.3 & 60 & & & Cn 655.10 & 60 \\
\hline Cn 655.17 & 90 & & & & & & \\
\hline Cn 655.18 & 100 & & & & & & \\
\hline Cn 719.1 & 40 & & & & & & \\
\hline \multirow[t]{2}{*}{ Cn 719.2} & 82 & & & & & & \\
\hline & 981 & + & 427 & $=$ & 1408 & : & 714 \\
\hline
\end{tabular}

Tab. 7: Männliche und weibliche Schafe im Vergleich zu alten Schafen aus ma-ro-pi.

\subsubsection{2. wo-ne-we ovIS ${ }^{\mathrm{m}}$}

PY

Cn 40

.1 wa-no-jo, wo-wo, pa-ro , ne-ti-ja-no-re, pa-ra-jo

$\mathrm{S} 4-\mathrm{H} 21$

2

wa-no-jo , wo-wo , pa-ro , po-so-pe-re-i , wo-ne-we

ovis $^{\mathrm{m}} 140$

wa-no-jo , wo-wo , pa-ro , zo-wi-jo , a-ko-so-ta-o

ovis $^{\mathrm{m}} 75$

.4

wa-no-jo , wo-wo , pa-ro, , po-ru-qo-ta , we-da-ne-wo

e-ko-me-no, pa-ro , pa-ta , pa-ra-jo

ovis $^{\mathrm{m}} 60$

.5

e-ko-me-no, pa-ro , [ ]ma-te-we, we-da-ne-wo

ovis $^{\mathrm{m}} 80$

.6

a-ne-u-te , pa-ro , ma-ri-ti-wi-jo , a-ko-so-ta-o

ovis $^{\text {th }} 70$

.7

ma-ro-pi , pa-ro , ro-ko, pa-ra-jo

ovis $^{\text {mi }} 83$

ma-ro-pi , pa-ro , ka-da-ro, we-da-ne-wo

ovis $^{\text {I }} 150$

.9

ma-ro , pa-ro , tu-ri-ta , a-ke-o-jo

ovis $^{\text {III }} 85$

re-pe-u-ri-jo , pa-ro , e-zo-wo , a[-ko-so-]ța-ọ

ovis $^{\text {min }} 80$

ดทัง ${ }^{\text {m }} 82$

ma-ro , pa-ro , ma-u-ti-jo , a-ko-șo-ta-o

ovis' $^{\prime} 60$

a-ne-u-te pa-ro , ka-ta-wa , a-ko-so-ta

ovis' 80

.14

a-te-re-wi-ja , pa-ro , e-wi-te-we , a-ke-o-jo

ovis $^{t} 70$

$.15-16$

vacant

PY

\begin{tabular}{|c|c|c|c|}
\hline Cn 643 & 1 & ạ-pạ-re-u-pi , pa-pa-ro, wo-ne-we & {$[.]^{\mathrm{m}}, 40, \mathrm{~S} 719-\mathrm{H} 1$} \\
\hline & .2 & wi-ja-wc-ra ${ }_{2}, a-k o-t e-u$ & $\operatorname{sus}^{t} 40$ \\
\hline & .3 & pi-*82, ma-ra-ni-jo , a-ke-o-jo & $\mathrm{CAP}^{\mathrm{m}} 48$ \\
\hline & .4 & pi-]*82, ku-ka-ra-so, a-ke-o-jo & $\operatorname{CAP}^{f} 53$ \\
\hline & .5 & ma-]ro-pi , pu-ma-ra-ko, we-da-ne-wo & $\mathrm{CAP}^{\mathrm{m}} 100$ \\
\hline & 6 & $\begin{array}{l}\text { ]ke-u, a-ke-ọ[-jo } \\
\text { desunt } 3 \text { versus }\end{array}$ & ] $\mathrm{CAP}^{t} 40$ \\
\hline
\end{tabular}


PY

\begin{tabular}{|c|c|c|c|}
\hline \multirow[t]{12}{*}{ Cn 719} & .1 & ma-ro-pi, ka-do-wo, a-ke-o-jo & ovIs $^{\mathrm{m}} 40$ \\
\hline & .2 & ma-ro-pi , to-si-ta , a-ke-o-jo & ovis $^{\mathrm{m}} 82$ \\
\hline & .3 & ma-ro-pi , me-ta-no, a-ke-o-jo & ovis $^{t} 60$ \\
\hline & .4 & pi-*82, ma-ra-ni-jo, pa-ra-jo & ovis $^{\mathrm{m}} 230$ \\
\hline & .5 & pi-*82, o-ku-ka, a-ke-o-jo & ovis $^{\mathrm{m}} 70$ \\
\hline & .6 & pi- $] * 82$, ra-mi-ni-jo, a-ke-o-jo & ovis $^{\mathrm{m}} 90$ \\
\hline & .7 & pi-*82 ] ku-pi-ri-jo , a-ke-o-jo & ovis $^{\mathrm{m}} 60$ \\
\hline & .8 & pi- $* 82$ ku-]ka-ra-so, a-so-ta-o & ovis'[ ] 30 \\
\hline & .9 & wi-]ja-we-ra ${ }_{2}$, ko-ru-no, pa-ra-jo & QYIS 66 \\
\hline & .10 & a-pa-]re-u-pi , pa-pa-ro, a-ko-so-ta- $Q$ & ovis $^{10} 100$ \\
\hline & .11 & wi-ja-we-ra ${ }_{2}$, a-ka-ma-wo , a-ko[-so- $]$ & ta-o ovis ${ }^{\mathrm{m}} 96$ \\
\hline & .12 & wi-ja-we-ra, a-ke-ta , wọ[-ne-]we & ovis $^{\mathrm{m}} 100$ \\
\hline
\end{tabular}

Das zweite in der PY Cn-Serie vorhandene Wort, das eine nähere Qualifikation von Schafen bietet, ist wo-ne-we (PY Cn 40.2, Cn 643.1, Cn 719.12). Es wurde von L. R. PALmer als *Fó $\rho v o v$ (,jung“) interpretiert. ${ }^{207}$ M. LANG dagegen schlug die Deutung als *Folvev́s (,dunkelfarben“) vor, womit also die Wollfarbe der Schafe beschrieben wurde. ${ }^{208}$ In beiden Fällen stünden die Schafe nicht als vollwertige Wolllieferanten zur Verfügung. Als „jung“ gekennzeichnete Schafe lieferten keinesfalls die erwartete Wollmenge und -qualität. Doch stellt sich die Frage, warum die Tiere von der Palastverwaltung dann überhaupt erfasst wurden. Wäre es nicht sinnvoller, die Schafe dann zu registrieren, wenn sie das für die Wollproduktion geeignete Alter erreicht hatten? Sollte es sich um Jungtiere handeln, die jedoch bereits geschoren wurden, wäre eine Angabe wie ki ne oder ne, bekannt aus den knossischen DSerien, ${ }^{209}$ oder WE (*wetalon) der Texte PY Un 138.3 und TH Wu 78, ${ }^{210} \mathrm{zu}$ erwarten. So scheint die Deutung als „dunkelfarbene“ Tiere durchaus zielführender. Dunkelfarbige Schafe waren auch in der klassischen Antike als Wolllieferanten weniger begehrt als jene mit weißem Wollkleid, da dunkle Wolle rauher und schwerer zu färben ist, wie durch „die häufigen Andeutungen der Alten über die Farbe der Wolle, die öftere Erwähnung der schwarzen, braunen oder röthlichen Schafe (Hom. Od. 4.135; 9.426), die fast ängstlichen Vorschriften zur Züchtung der weißen, die öfter aufgezeichneten Rückschläge zu dunklen oder fleckigen oder fahlen Lämmern ... “ dargelegt wird. ${ }^{211}$ Die dunklen Tiere wurden dabei, wenn sie nicht speziell wegen der dunklen Farbe gezüchtet wurden, als „Knechtsvieh“ an die Hirten abgegeben. ${ }^{212}$ Könnte dies auch für die als wo-ne-we charakterisierten Schafe der pylischen Cn-Serie zutreffen? Standen diese Tiere also dem Palast nicht für die Wollproduktion zur Verfügung und wurden deshalb als „Abgang“ verzeichnet? Es ist durchaus vorstellbar, dass diese Tiere von der Palastverwaltung entweder zur Schlachtung freigegeben wurden oder auch in den Besitz der „Hirten“ übergingen, die für deren Aufzucht und Betreuung verantwortlich gewesen waren. Wir dürfen wohl annehmen, dass auch die mykenischen Paläste weiße Wolle zur Verarbeitung bevorzugten. Möglicherweise wurden sie nicht für die Zucht he-

\footnotetext{
${ }^{207}$ L. R. Palmer 1963, 464.

${ }^{208}$ Lang 1966, 258: „,dark-fleeced sheep“; Ilievski 1968, 622 u. Fn. 32: „wine-coloured“; Doria 1958, 24f: „vinai“.

${ }^{209}$ Killen 1968, 121.

${ }^{210}$ Piteros-Olivier-Melena 1990, 161.

${ }^{211}$ Magerstedt 1859, 110; s. auch Kroll 1924, 596.

${ }^{212}$ Kroll 1924, 596.
} 
rangezogen, lieferten jedoch immer noch Fleisch, Milch aber auch die wohl geringer geschätzte „dunkelfarbene“ Wolle. Diese Produkte konnten entweder vom Palast selbst verwertet werden, oder man gab die Tiere an die „Hirten“ ab. Jedenfalls ist auffällig, dass alle als wo-ne-we („dunkelfarben“) gekennzeichneten Schafe mit dem Ideogramm ovis ${ }^{\mathrm{m}}$ registriert wurden. Es könnte wohl - analog zu den als pa-ra-jo (,alt“) definierten Tieren ${ }^{213}$ - angenommen werden, dass hinter dem Ideogramm ovIS ${ }^{\mathrm{m}}$ sowohl männliche als auch weibliche Tiere zu vermuten wären, ${ }^{214}$ die für die Wollproduktion nicht interessant waren und deshalb auch nicht mehr nach Geschlechtern getrennt erfasst wurden.

\subsubsection{3. „Collectors“/,Besitzer“, „Hirten“ und Herden}

Der Terminus a-ko-ra ist in der pylischen Cn-Serie jeweils mit einem Personennamen im Genetiv verbunden: a-ko-so-ta-o a-ko-ra (Cn 453), a-pi-me-deo a-ko-ra (Cn 655.5), we-da-ne-wo a-ko-ra (Cn 655.6.13), a-ke-o-jo a-ko-ra (Cn 655.11.12); auf Cc 660.a ist dagegen $a-k e-o$ a-ke-re zu lesen. Der Begriff

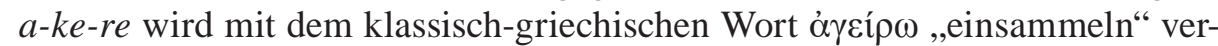
bunden, und auch die Ableitung a-ko-ra darf in diesem Sinne verstanden werden. ${ }^{215}$ Aufgrund der Schreibung der Personennamen in Zusammenhang mit $a$-ko-ra/a-ke-re wurden die vier Männer a-ko-so-ta, a-pi-me-de, we-da-ne-u und $a-k e-o$ als „Collectors“ bezeichnet. ${ }^{216}$ Diese Personen sind aufgrund ihrer Nennung auf weiteren Linear B-Tafeln zur höheren Gesellschaftsschicht des mykenischen Reiches von Pylos zu rechnen. ${ }^{217}$

213 Siehe S. 46.

214 Halstead 1990-91, 354 Fn. 32.

215 Ventris-Chadwick 1973, 200, 434, 529; Ilievski 1968, 618; Carlier 1992, 160; Bennet 1992, 68.

216 Ventris-Chadwick 1973, 434, 529

217 A-ke-o ist neben Cc 660.a, Cn 655.11.12 und Cn 643.3.4.6 noch in weiteren Texten der CnSerie (Cn 40.10.14, Cn 45.5.8.11.12, Cn 254.6, Cn 599.1.3.4.6, Cn 600.5.6.11, Cn 702.3.4, Cn 719.1-.3.6.7) als „Besitzer“ von Tierherden zu finden. Auf An 192.14 wird $a$-ke-o als ka-wi-jo definiert und scheint eine höhere Position inne gehabt zu haben (Lindgren 1973 II, 75, 178; Aura Jorro 1985, s.v. ka-wi-jo). Der Name a-ko-so-ta ist auf PY An 39v.5 mit Truppen verzeichnet, auf Cn 40.5, Cn 45.3, Cn 453.1, Cn 599.2, Cn 702[.1], Cn 719<.8>.10[.11] mit Tierherden, im Landverteilungstext Eq 213.1 (o-wi-de $a$-ko-so-ta) als Inspektor, auf Un 267.1 (o-do-ke a-ko-so-ta) als Zulieferer für die Salbenproduktion und auf Pn 30.1, Va 482, Xn 435.2 sowie Wa 917. Zur Interpretation des a-ko-so-ta s. Aura Jorro s.v. Der auf Cn 655.5 mit $a$-ko-ra verbundene $a$-pi-me-de ist in den Landverteilungstexten Eb 473.1, Eb 539[.14] verzeichnet und hat nach Ausweis der Eb-Serie auch drei do-e-ro unter seiner Ägide (Eb 1186.1, Eb 1187.1, Eb 1188.1, Ep 539.10.11.12). Dieser a-pi-me-de scheint nach Eb 473.1.2 in Verbindung mit Ep 539.14 ein e-qe-ta gewesen zu sein (Lindgren 1973 II, 47, Deger-Jalkotzy 1978, 63f.). Der letzte der vier „Collectors“, we-(u-)da-ne-u, findet sich auf den Tafeln PY An 610.14 mit Truppen von Ruderern, auf Cn 40.4.6.9, Cn 45.2.3.4, Cn 254.4a.5.7, Cn 600.8, Cn 643.5, Cn 655.6.13-.20 mit Tierherden und in der PY Es-Serie als Empfänger von Getreide (Es 644.6, Es 645.3, Es 648.3, Es 650.6, Es 651.3, Es 652.3, Es 653.3, Es 703.1.3, Es 726.3, Es 727.3, Es 728.3, Es 729.3). Weiters ist der Name we-da-newo auf den Tafeln PY Na 856 und Na 1041 mit Flachs (SA) verbunden. We-u-da-ne-u hatte auch Lieferungen von Tieren an den Palast von Pylos zu tätigen (Cn 418) und ist auf PY Un 1193 mit der Angabe DA zu finden. Von M. Lindgren wurde sogar vorgeschlagen weu-da-ne-u als den lāwāgetās des Reiches von Pylos zu sehen - Lindgren 1973 II, 187. Diese Interpretation hat sich allerdings nicht durchgesetzt. Zweifellos hatte we-u-da-ne-u jedoch einen hohen Rang in der pylischen Hierarchie inne. 


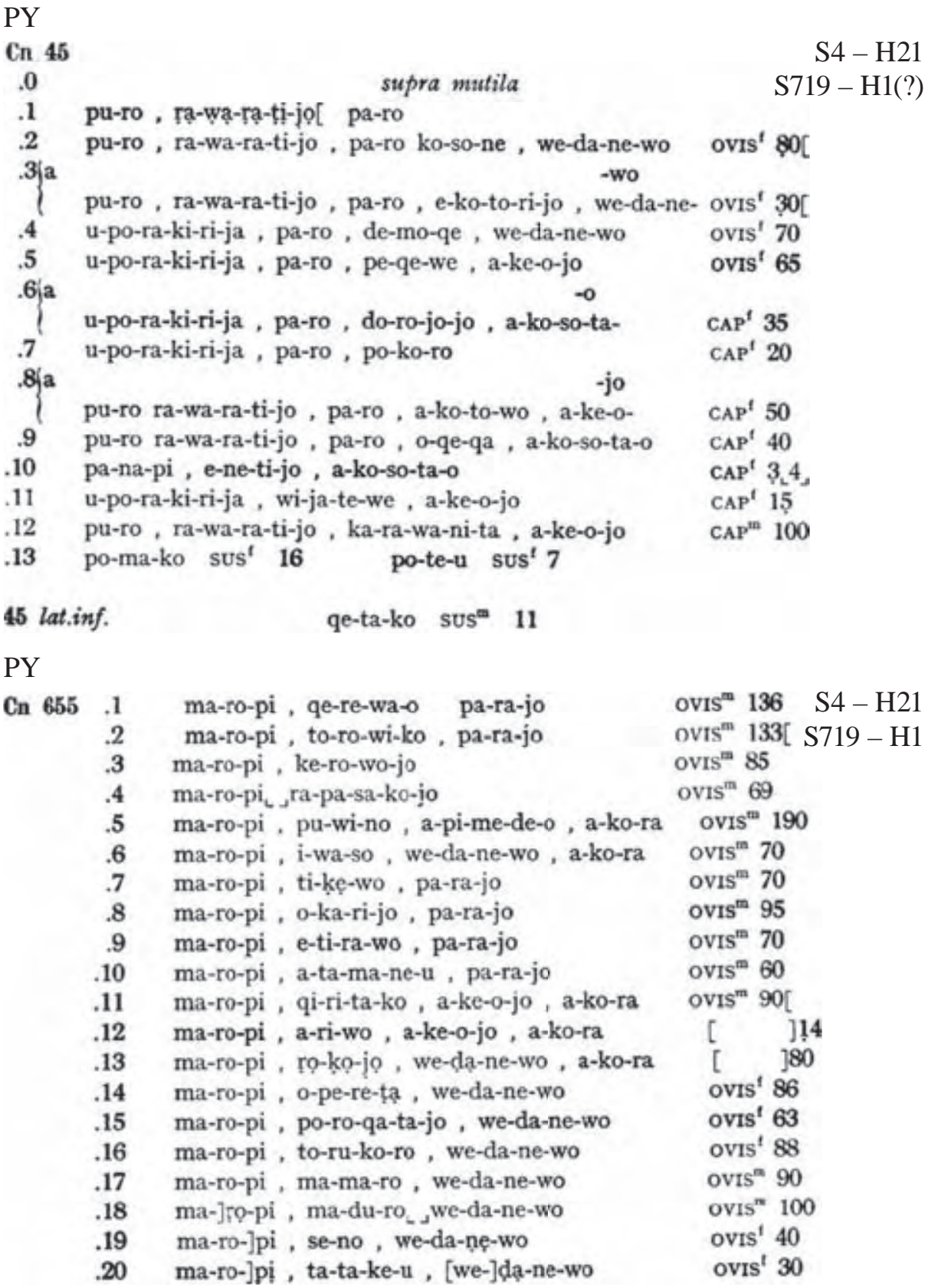

Die immer wieder durchgeführte Diskussion zur Interpretation derjenigen Personen, die als „Collectors“ bezeichnet werden, muss vor allem im Zusammenhang mit der Tierhaltung durchgenommen werden. Die knossischen D-Serien enthalten, neben den Schafideogrammen, ähnliche Eintragungen wie die pylische Cn-Serie: Toponym, Personenname mit pa-ro (= „Hirten“-Name) und Personenname im Genetiv („Collector“-Name). Im Gegensatz zu den pylischen Texten finden sich jedoch auch Aufzeichnungen die keinen „Collector“-Namen aufweisen. Aufgrund dieser Tatsache schlug L. R. PALMER vor, dass die „Collectors“ die mit ihren Namen versehenen Herden besaßen, während solche ohne Namen eines „Collector“ dem Palast beziehungsweise dem wanaks gehörten. ${ }^{218}$ In Documents in Mycenaean Greek wurde diese Interpretation etwas modifiziert: der Palast besitzt alle Herden, aber er überantwortet manche Tiere an

${ }^{218}$ L. R. Palmer 1957, 570; Olivier 1967, 79; Olivier 1972, $23 f ., 27$. 
Mitglieder der Nobilität des Reiches zu deren Unterhalt. ${ }^{219}$ J. T. KILLEN wandte für die als „Collector“ bezeichneten Personen, aufgrund der Interpretation L. R. PALMERS, den Begriff „Besitzer“ an.220

Bis auf einige wenige Tiere - pa-ra-jo ovIS ${ }^{\mathrm{m}}$, wo-ne-we ovIS ${ }^{\mathrm{m}}$ und solche ohne „Collector"-Namen - sind die Schafe und Ziegen der Texte PY Cn 40, Cn 45, Cn 254 + Cn 272, Cn 599, Cn 600, Cn 643, Cn 655, Cn $702[+]$ 1461 und Cn 719 offensichtlich alle im Besitz der vier „Collectors“. Dazu zählen auch die zusätzlich mit $a$-ko-ra definierten Schafe, welche vom Palast von Pylos ebenfalls an die „Collectors“ vergeben wurden. Die der Bedingung $a$-ko-ra/a-ke-re unterliegenden Schafe der pylischen Cn-Serie (Cn 453, Cn 655.5.6.11.12.13, Cc 660) standen somit möglicherweise dem Palast von Pylos nicht zur Wollproduktion zur Verfügung. Da die „Collectors“ auch als die Besitzer der sonstigen Schafe und Ziegen zu sehen wären, die in den Texten mit ihren Namen verbunden sind, könnte angenommen werden, dass es sich bei diesen Tieren ebenfalls um eine Art „Versorgungsgüter“ handelte, die Angehörigen der pylischen Aristokratie vom Palast zur Verfügung gestellt wurden. ${ }^{221}$ Es ist doch auffällig, dass die vier „Collectors“ der pylischen Cn-Serie ausschließlich Schafe und Ziegen - also keine Rinder und Schweine - erhielten, die Wolle, Haare und Milch liefern konnten.

Die an die „Collectors“ vergebenen Herden stammten ursprünglich aus dem Besitz des Palastes von Pylos. Dies ist schon alleine deswegen ersichtlich, da die Palastverwaltung die Aufzeichnung der Tiere vornahm. Aufgrund der Nennung von „Hirten“-Namen wurden die Schafe und Ziegen offensichtlich aus deren Bestand abgegeben. Und für den Palast von besonderem Interesse dürfte dabei gewesen sein, dass die an die „Collectors“ verteilten Tiere somit nicht mehr als Wolllieferanten für die palatiale Textilindustrie Palast zur Verfügung standen.

Markant und immer wieder angeführt, ist der Unterschied der Anzahl der „Collectors“/,Besitzer“ der pylischen Cn-Serie und der knossischen D-Serien. In den knossischen $\mathbf{D}$-Serien finden sich die Namen der in folgender Tabelle (Tab. 8) aufgelisteten Personen, die als „Besitzer“ definiert werden können. ${ }^{222}$

Dazu kommen noch die Angaben von $e-m a-a_{2}-O$ („,des Hermes“) $)^{223}$ auf KN D 411 und po-ti-ni-ja-we/-(i)-jo (,zur Potnia gehörig“) auf KN Dl 930.A, DI 933.A, Dl 946.A, Dl 950.A, Dl 7147.A, Dl 7503.A. ${ }^{224}$

a-di-je-wo auf D 747.a, D 5520.a;

$a-k a-i-j o$ auf De 1084.a, Dv 1085.a;

$a-k a-t a-j o$ auf Dv 1086.a, Dv 1331.a;

a-no-qo-ta auf Da 1323.a, Dq 45.a, D 7334.a;

$a-t e$-jo auf Da 1392.A, Db 1329.a, Dc 1303.A,

Dc 1337.A, Dc 5687.A, De 1301.A, De 1307.A; da-mi-ni-jo auf Da 1116.a, Dc 1117.A, Dc 1118.A;

$e$-me-si-jo auf De 1381.A;

$e$-se-re-e-jo auf Dl 947.A, Dl 949.A;

ka-to(-ro) auf KN Do 1054.b, Do 7613.b, Do 7740.b, te-ra-po-si-jo auf Da 1314.a, De 1361.A, De 1371.A; pe-ko auf Dc 8080.A, Dv 7098.A, Dv 1621.a;

pe-ri-qo-ta/pe-ri-qo-te-jo auf Da 1172.B, Da 1321.B,

Da 1333.B, Dn 5668 (]qo-te-jo), Dq 42.a, Dq 46.A,

Dq 8351.a;

]ra-to-jo auf Dd 1342.A;

ra-wo-qo-no auf Dl 928.A, Dl 9841.1 (]-qo-no-jo), D 1650.a;

sa-qe-re-jo auf Dl 412.A, Dl 794.A, Dl 935.A, Dl 940.A, Dl 944.A;

219 Ventris-Chadwick 1973, 434; Killen 1995, 213.

220 Killen 1976, 118; Palmer 1957, 570; Killen 1996, 77; Killen 1979, $176 f f$.

221 Killen 1995, 213f.

222 Zur Auflistung dieser Personen als „Besitzer“ s. Rougemont 2001, 131 Tab. 1; für eine genauere Unterteilung nach den einzelnen Texten der D-, Da-Dv-Serien s. Olivier 2001, 142ff.; s. auch Ilievski 1992, 347f. Appendix II.

223 Nosch 2000, 214f.

224 Vgl. Olivier 2001, 142ff.; Ilievski 1992, 347f. Appendix II. 
Dq 438.b, Dq 686.b;

ke-u-po-da auf Dq 442.a;

ko-ma-we-(to/-te) auf Dk 920.a, Dk 931.a, Dk 1049.a,

Dv 1272.A, Dv 7176.a, Dv 8562.B;

]ko-ta-o auf Dq 7126.a;

]ko-we-jo auf Dk 925, Dq 445.a;

ku-ru-me-ni-jo auf Da 1173 lat. inf.;

o-pa-we[ auf Dv 1434.a;

$o$-re-te-wo auf Dq 439.B, Dq 441.B $u$-ta-jo auf Da 8228.a, De 1138.A, De 1141.B,

De 1383.A, De 5336.B, De 7203.A, Dv 1133.a,

Dv 1139.a, Dv 1142.A, Dv 1146.A, Dv 1154.a, Dv 1370.a, Dv 1417.B;

we-we-si-jo auf Db 1155.A, Db 1159.A, Db 1160.A,

Db 1165.A, Db 1166.A, Db 1168.A, Dd 1579.A,

De 1151.A, De 1152.A, De 1153.A;

wi-jo-qo-ta auf Dq 1026.a, Dq 7852.a;

Tab. 8: Die Namen der „Besitzer“ in den knossischen D-, Da-Dv-Serien.

Nicht zu leugnen ist, dass es sich ebenfalls um Männer gehandelt hat, die eine hohe Stellung innerhalb der Hierarchie des Palastes von Knossos innehatten. Dies zeigt sich durch das Vorkommen ihrer Namen in Aufzeichnungen der unterschiedlichen Bereiche der palatialen Wirtschaft. Allerdings hat es den Anschein, dass sich die gehobene Stellung hauptsächlich durch die Schafhaltung und Textilindustrie begründet. ${ }^{225}$ Die „Besitzer“ gehörten nach P. HR. ILIEvSKI jedoch zur „ruling class“ der knossischen Gesellschaft. ${ }^{226}$ Wie bereits besprochen erhielten vier Angehörige der pylischen Aristokratie (we-da-ne-u, a-koso-ta, a-ke-o, a-pi-me-de) vom Palast von Pylos anscheinend Versorgungsgüter in Form von Tierherden, die in der PY Cn-Serie verzeichnet wurden. ${ }^{227}$ Dies scheint für die knossischen D-Serien aufgrund des Interesses des Palastes an der produzierten Wolle ausgeschlossen zu sein. Hätte er die Tiere als Versorgungsgüter vergeben, wäre eine detaillierte Aufzeichnung der Wollmenge (vor allem der fehlenden) sinnlos gewesen. Wir müssen also davon ausgehen, dass die Aufzeichnungen der KN D-Serien aus einem anderen Grunde erfolgten als jene der PY Cn-Serie.

Dem Palast von Knossos ging es um die Wollproduktion und damit verbunden um die wollproduzierenden Schafe. ${ }^{228}$ Die immer wieder gestellte Frage lautet, warum die D-Serien zusätzlich zu den „Hirtennamen“ Personennamen im Genitiv enthalten. L. R. PALMER sah in diesen Personen, diejenigen, die vom Palast die Tiere als Besitz erhielten. ${ }^{229}$ Diese Definition wurde von J. T. KILLEN aufgegriffen und er nannte die Männer „owners“. ${ }^{230}$ Der Palast gab also einen Teil seiner Herden an „Besitzer“ ab. Doch hatte er damit immer noch Interesse an dem erwarteten Wollertrag, also auch an den Schafherden. Wäre es nicht denkbar, dass der Palast von Knossos seine Herden, nicht direkt in den Besitz, sondern in die Verantwortung von Einzelpersonen gegeben hatte? ${ }^{231}$ Die Tafeln zeigen, dass nur ein Teil der palatialen Herden an „Besitzer“ vergeben war. ${ }^{232}$ Der Palast hatte jedoch weiterhin großes Interesse an diesen Schafen, was daran zu erkennen ist, dass über die Tiere detaillierte Aufzeichnungen geführt wurden. ${ }^{233}$

Die „Besitzer“ waren sowohl für die Schafhaltung als auch die Wollproduktion verantwortlich. Ein ähnliches System findet sich im Alten Orient, das

\footnotetext{
225 Vgl. Rougemont 2001, 132; Olivier 1967, 84; Bennet 1992, 96;

${ }^{226}$ Ilievski 1992, 323.

${ }^{227}$ Siehe S. 49.

${ }^{228}$ Dies konnte von J. T. Killen ausführlich dargelegt werden, s. Killen 1964, 1ff.

${ }^{229}$ L. R. Palmer 1957, 570.

${ }^{230}$ Killen 1976, 118.

231 Siehe dazu Carlier 1992, 160ff., 164.

232 Olivier 1967, 71ff.; Bennet 1992, 85ff.

233 Vgl. Killen 1995, 213; Carlier 1992, $160 f$.
} 
sogenannte Palastgeschäft. ${ }^{234}$ So schrieb J. RENGER jüngst in seinem Artikel Das Palastgeschäft in der Altbabylonischen Zeit sich selbst zitierend: „... economically viable activities of institutional households tended to be granted to members of the élite or upper classes as franchises, a form of economic management termed by F. R. Kraus as ,Palastgeschäft'. The overriding purpose was to relieve institutional households of the high cost of maintaining a large laborforce that was in many cases in fact only needed for seasonal work“. 235 Der Palast übertrug sogenannten Entrepreneuren („Mittelsmännern“), die zur höheren Gesellschaftschicht gehörten, verschiedene wirtschaftliche Aktivitäten und die damit verbundenen Risken, wobei sich hauptsächlich drei Formen des Palastgeschäftes unterscheiden lassen:

„-- Palastgeschäfte, die auf landwirtschaftlicher Produktion oder Viehhaltung beruhen,

- Palastgeschäfte, in denen es um das Ausbeuten natürlicher Ressourcen geht, und schließlich

- Palastgeschäfte, deren Aufgabe im Bereich der Dienstleistungen liegen, die teilweise mit den beiden zuvor genannten Formen von Palastgeschäften verbunden sein können“ “ ${ }^{236}$

Bei der Kleintierhaltung stand dabei die Wollproduktion im Vordergrund, und erst in zweiter Linie ging es um die Bereitstellung von Schlachtvieh, während bei der Rinderhaltung die Versorgung mit Arbeitstieren ausschlaggebend war. ${ }^{237}$ Für die Kleinviehwirtschaft hieß dies, dass der Palast größere Schafherden an „Oberhirten“ vergab. ${ }^{238}$ Die jeweiligen Herden wurden in kleineren Einheiten an „Hirten“ vergeben, und davon wiederum kleinere Teilherden an „Unterhirten“. Diese „Unterhirten“ waren für das tatsächliche Hüten der Schafe verantwortlich. Doch hatten auch die „Oberhirten“ und „Hirten“ Herden, die offiziell direkt von ihnen gehütet werden sollten. ${ }^{239}$ In der Praxis wurden dafür jedoch ebenfalls einfache Hirten eingesetzt. ${ }^{240}$ Das eben dargelegte System veranschaulicht die nachfolgende Tabelle (Tab. 9).

Inwieweit lässt sich das mesopotamische System des Palastgeschäftes mit den Aufzeichnungen der knossischen D-Serien vergleichen? Die folgenden Ausführungen $^{241}$ sollen einzelne Aspekte der Linear B-Aufzeichnungen darlegen, die durchaus Hinweise auf Organisationsformen in der Tierhaltung liefern können, die Ähnlichkeiten zum orientalischen Palastgeschäft aufweisen. ${ }^{242}$

Zunächst einmal standen beim orientalischen Palastgeschäft in Bezug auf die Schafe die Viehzucht und die damit verbundene Wollgewinnung im Vordergrund. ${ }^{243}$ Die Bedeutung der Wollgewinnung dürfen wir nach den Texten aus Knossos, auf denen genau das Geschlecht, das Alter, das Fehlen von Schafen und der Wollertrag verzeichnet wurden, für diesen Palast ebenfalls annehmen. ${ }^{244}$

${ }^{234}$ Zum orientalischen Palastgeschäft s. Renger 2000, 153ff., zur Viehhaltung bes. S. 163f.; s. auch Postgate 2001, 181ff., insbesondere 187ff.

${ }^{235}$ Renger 2000, 154 u. Fn. 6.

${ }^{236}$ Renger 2000, $154 \mathrm{f}$.

${ }^{237}$ Renger 2000, 155.

${ }^{238}$ Renger 2000, 163; Postgate 2001, 189 verwendete stattdessen den Begriff „herding-contractor“; s. ferner van de Mieroop 1993, 168; van Driel 1993, 219.

239 Eine anschauliche graphische Darstellung des Ablaufes des Palastgeschäftes in Bezug auf die Viehhaltung findet sich bei Renger 1998, 193.

${ }^{240}$ Renger 2000, 163; van Driel 1993, 221, 225.

${ }^{241}$ Siehe auch weiter unten, S. 59ff.

242 Vgl. Carlier 1992, $161 f$.

${ }^{243}$ Renger 2000, 163.

${ }^{244}$ Killen 1964, 5, 8. 


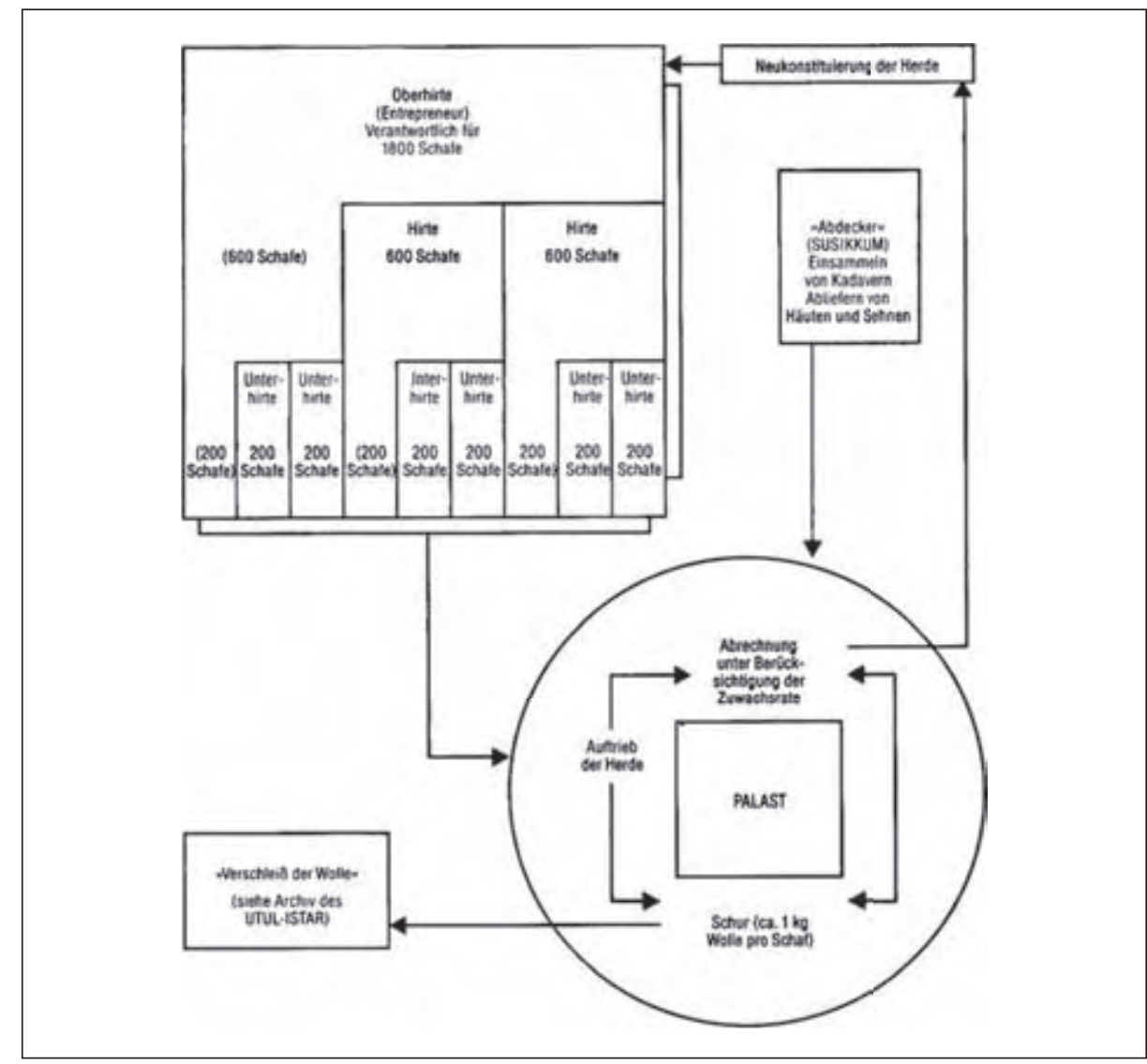

Tab. 9: Orientalisches Palastgeschäft in Bezug auf die Tierhaltung (Abb. nach Renger 1998, 193).

Nach den KN D-Serien zu urteilen, gab es Tiere, die an einzelne Personen (die sogenannten „Besitzer“) vergeben waren. ${ }^{245}$ Dies wird dadurch begründet, dass jeder einzelne Name mit mehreren Herden verbunden werden kann, die an verschiedenen Orten und bei unterschiedlichen „Hirten“ waren. Die knossischen „Besitzer“ sind demzufolge als die Hauptverantwortlichen für diese Schafherden zu sehen. Gleichzeitig sind die Namen dreier dieser Männer auch als „Hirtennamen“ verzeichnet - a-no-qo-ta (KN Da 1289.B), ka-to-ro (KN Dv 5287, Dv 1169.B), wi-jo-qo-ta (KN Db 1305.B). ${ }^{246}$ Sollte es sich in diesen Fällen tatsächlich um dieselben Personen handeln, ließen sich die „Besitzer“ der knossischen D-Serien durchaus mit den „Oberhirten“ des mesopotamischen Palastgeschäftes vergleichen. ${ }^{247}$ Sie hätten also die Verantwortung für einzelne Schafherden vom Palast übertragen bekommen, die sie entweder unter die Aufsicht von „Hirten“ stellten (a-na-qo-ta auf KN Da 1323.a, D 45.a, D 7334.a; ka-to-ro auf KN Do 1054.b, Do 7613.b, Do 7740.b, Dq 438.b, Dq 686.b; wi-joqo-ta auf Dq 1026.a, Dq 7852.a) oder theoretisch selbst betreuten (Da 1289.B, Db 1305.B). Im Falle von ka-to-ro könnte dieser zusätzlich Herden von einem anderen „Besitzer“ (KN Dv 1169.B von we-we-si-jo) übernommen haben.

Einige der „Besitzer“-Namen finden sich des Weiteren auf knossischen Texten, die anderen Wirtschaftszweigen zuzuordnen sind (Tab. 10). ${ }^{248}$ So sind

\footnotetext{
${ }^{245}$ Siehe S. 53.

${ }^{246}$ Ilievski 1992, 322.

247 Vgl. Carlier 1992, 164.

248 Vgl. van Driel 1993, 222, 225 (für Uruk). Bei den „Hirten“ aus Pylos lässt sich feststellen, dass es sich um Personen mit unterschiedlichen Berufen handelte, die zusätzlich die Ver-
} 
manche Namen zusätzlich in Aufzeichnungen unterschiedlicher Inhalte, vor allem jedoch jenen der Textilproduktion, anzutreffen. ${ }^{249}$ Natürlich müssen nicht alle gleichlautenden Namen tatsächlich denselben Personen eigen gewesen sein, doch kann das Vorkommen von Personennamen aus Texten der Schafzucht beziehungsweise der Wollproduktion in solchen anderer Tätigkeitsfelder darauf hindeuten, dass sich zumindest einige der „Besitzer“ nicht nur auf die Schafzucht beschränkten. Eine Auflistung des Vorkommens der „Besitzer“ auf Tafeln der knossischen A-, L- und sonstiger Serien bietet J.-P. OLIVIER in seinem Artikel Les „collecteurs“: leur distribution spatiale et temporelle. ${ }^{250}$ Aus diesem sind die folgenden Angaben (Tab. 10) in Bezug auf einige Namen der oben genannten Besitzer ${ }^{251}$ übernommen, soweit sie sich anderen Bereichen der mykenischen Wirtschaft und bestimmten „Aktivitäten“ zuordnen lassen.

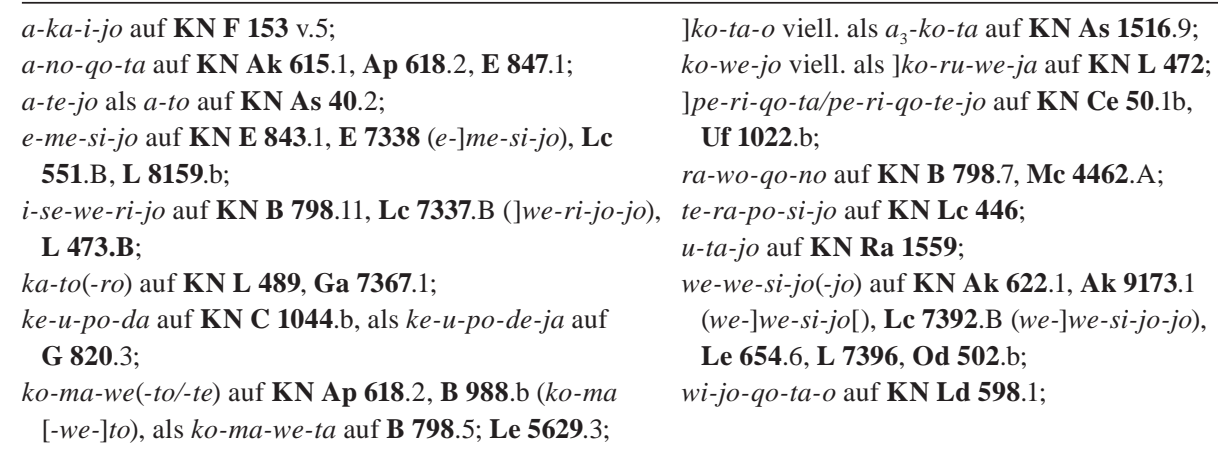

Tab. 10: Das Vorkommen der Namen knossischer „Besitzer“ in weiteren Texten aus Knossos.

Die „Hirten“ der knossischen D-, Da-Dv-Serien standen offensichtlich im Dienste des Palastes von Knossos (Herden ohne „Besitzernamen“), finden sich jedoch auch in Aufzeichnungen zusammen mit „Besitzern“. Somit muss es sich bei diesen „Hirten“ um Personen handeln, die in der Herdenverwaltung eine Stufe niedriger standen als die „Besitzer“, da sie sonst wohl kaum die Verantwortung über deren Herden übernommen haben würden. Ich spreche hier nicht vom Hüten der Schafe, da ich denke, dass sich diese Männer mit den „Hirten“ des orientalischen Palastgeschäftes vergleichen lassen. Nach P. Hr. ILIEvSKI sind die „Hirten“ der knossischen Texte Personen, die der gesellschaftlichen Mittelschicht zuzurechnen sind: „While the names of the «collectors», men belonging to the ruling class, with the exception of a few of them (about 5 out of 25) are easily recognizable as Greek, the case with the names of the "shepherds", people who belonged to a social class of middle rank, is the opposite. According to an approximate calculation more than one half of them (200, i. e. about $57 \%$ ) are without identification. A large number of them are very likely of non-Greek origin, born of the inhabitants of the pre-Greek population which continued to dwell in Crete together with the conquerors, the Mycenaean Greeks“. ${ }^{252}$ Die mesopotamischen „Hirten“ jedenfalls waren für die ihnen anvertrauten Schafe zwar verantwortlich, theoretisch hatten sie die Schafe selbst zu betreuen, gehütet wurden die Tiere jedoch von den „Unterhirten“. ${ }^{253}$ Inwie-

antwortung für palatiale Herden übernahmen. Siehe S. 63ff u. Tab. 12. Siehe ferner Carlier 1992, 163f.

${ }^{249}$ Killen 1995, 213.

${ }^{250}$ Olivier 2001, $142 \mathrm{ff}$.

251 Siehe S. 51ff.

252 Ilievski 1992, 323.

253 Siehe Tab. 9; Renger 1998, 193; van Driel 1993, 225. 
weit die „Hirten“ der knossischen Serien tatsächlich mit den Schafen auf die Weide zogen oder diese möglicherweise an „Unterhirten“ vergleichbar zum orientalischen System weitergaben, lässt sich anhand der knossischen Aufzeichnungen nur indirekt erschließen. ${ }^{254}$ Zumindest ist augenfällig, dass die den „Hirten“ übergebenen Tiere für verschiedene Lokalitäten verzeichnet sind und es ist kaum anzunehmen, dass ein einziger Mann, also der „Hirte“, die Schafe an mehreren Orten gleichzeitig selbst hüten konnte. Eine Aufteilung und Weitergabe der Schafherden an Personen („Unterhirten“), die tatsächlich das Hüten übernahmen, ist wahrscheinlich. Dies würde den „Hirten“ auch genügend Freiraum lassen, sich anderen Tätigkeiten zu widmen. Manche dieser „Hirten“ waren vielleicht - gleich den „Besitzer“ - in andere Wirtschaftszweige des knossischen Palastes involviert. Zumindest finden sich einige der „Hirtennamen“ auch in anderen Serien aus Knossos verzeichnet (Tab. 11). ${ }^{255}$ Erfasst wurden in dieser Auflistung nur diejenigen Namen, die eindeutig einem anderen Tätigkeitsbereich als der Schafhaltung zugeordnet werden können. Auch wenn dies noch kein Beweis dafür ist, dass es sich um dieselben Männer handeln muss, könnten aber doch einzelne Personen unterschiedlicher Professionen zusätzlich die Verantwortung über palatiale Herden übernommen haben. ${ }^{256}$

a-ka-to von Dv 5256 auf Sc 256;

$a-k e-u$ von De 7096, Dv 1133 auf Ap 628;

$a-m e-a$ von Da 1189 auf F 153.3, Od 765;

$a-r a-k o$ von Db 1236, De 1307 auf As 607;

$a-r a-s i-j o$ von Df 1229 auf Fh 369;

$a-r e-t a_{2}$ von Df 1325 auf X 7556;

a-te-mo von Dc 1298, De 1648 auf As 1520.3;

a-*56-no von Dv 5232 auf As 1520.13;

]da-nwa-re von Db 1302 auf Sc 5058;

di-ka-ta-ro von Dd 2010 auf As 566.1;

di-ka-ta-so von Dl 916 auf Ga 427.1;

di-wo von Dv 1503 auf E 842.1;

di-za-so von Dv 1505 auf Pp 493;

du-ni-jo[ von Dv 5690 auf As 1516.21;

du-to von Dc 8354 auf As 1516.14;

e-ke-me-de von Dd 659 auf U 4478.18;

e-ru-to-ro von Dk 1074 auf As 1517.7;

e-u-na-wo von Dv 1206 auf As 1520.9;

$i-n e-u$ von Da 1379 auf As 607.5;

]ka-mo von Db 1263, Dq 440 viell. auf As 604;

ko-ri-jo von Db 1267 auf Nc 8146;

ko-so von Dv 8383 auf As 40.5, Ap 637.2;

ki-ta-ne-to von Da 1108 auf Am 821;

ma-di von Db 1168 auf KN As 603.2;

ma-di-qo von Db 1460, Dl 930 auf B 806.4;

mi-ru-ro von Da 1127 auf As 1516.5;

no-da-ro von Dc 1228 auf As 609.3; $o-k u$ von Da 1170, Dl 792 auf As 8161;

pa-]qo-si-jo von Dq 441 auf B 988;

$p a-t i$ von Dd 1281 auf As 1516.17;

]pu-to-ro von Da 1333 auf As 1516.9;

]qa-ra-su-ti-jo von Dd 1150, Dv 8193 auf Nc 4489; qe-da-do-ro von De 1294 auf Uf 121;

qe-ro von Db 1204 auf As 602.3;

qo-te-ro von Da 1495 auf As 1516.8;

re-me-to von Dd 1106 auf L 771.2, Pp 495;

ri-wi-so von Da 1114 auf Ga 419.2, Uf 111;

sa-ma-ri-jo von Da 1147 auf Np 857;

sa-ma-ri-wa-ta von Dv 1188 auf As 645.2;

sa-zo von Dv 5301 auf As 1520.10;

si-da-jo von Dl 947 auf Nc 4490;

si-ra-ko von Db 8352 auf As 5976.1;

ta-so von Dv 5200 auf As 608.2;

ta-za-ro von Db 1097 auf Ch 896;

te-jo von Dv 7617 auf L 565.1;

]te-ru-ro von Da 1380 auf Mc 4464.A;

te-wa-jo von Dl 7503 auf Ce 156.2;

to-u-na-ta von Dv 1479, Dm 1182 auf B 803.1;

u-ra-mo-no von Da 1315 auf As 1516.6;

we-wa-do-ro von Dv 1601 auf Sc 252;

wi-ja-ma-ro von Da 1378 auf As 1516.21;

wi-je-mo von Dv 1266 auf As 609.2;

wi-ra-ne-to von Dv 1205, Dv 1457 auf As 1516.22; ze-ro von Da 5218 auf As 4493.3;

Tab. 11: Das Vorkommen der Namen der knossischen „Hirten“ in weiteren Texten aus Knossos.

Die verschiedenen Berufe schließen „privaten“ Besitz von eigenen Tieren nicht aus, wenn man bedenkt, dass der Viehbesitz zum Beispiel in der klassischen Zeit durchaus ein Zeichen von Reichtum war. Eines ist jedoch ersichtlich, dass

${ }^{254}$ Vgl. dazu S. 65ff. über die pylische Ae-Serie.

${ }^{255}$ Siehe Ilievski 1992, 339ff.

${ }^{256}$ Vgl. dazu im Folgenden die „Hirten“ der pylischen Cn-Serie, S. 59ff. 
nämlich der Palast von Knossos nicht alle Schafherden an „Besitzer“ („Oberhirten“) vergab, sondern den größten Teil sogar direkt an „Hirten“. 257

Die „Hirten“ der KN D-Serien waren Angehörige der Mittelschicht der knossischen Gesellschaft, die für die ihnen anvertrauten Schafe zwar verantwortlich waren, das Hüten aber wohl sogenannten „Unterhirten“ überließen. Dies lässt sich daran erkennen, dass die den „Hirten“ übergebenen Tiere sich an verschiedenen Lokalitäten befanden, an denen sich der „Hirte“ nicht gleichzeitig befunden haben kann. Er musste also für die Betreuung der Tiere andere Personen einstellen.

Als letztes sei hier angefügt, dass auch Texte erhalten sind, die den Gott Hermes (KN D 411) und die Göttin Potnia (po-ti-ni-ja-we/-(i-)jo „zur Potnia gehörig“258 in der KN Dl-Serie) als „Besitzer“ verzeichnen. ${ }^{259}$ Sollte dies heißen, dass der Palast auch an Gottheiten respektive deren Heiligtümer beziehungsweise Kultpersonal Schafherden, vergleichbar zum orientalischen Palastgeschäft, vergab? Im Falle des Textes KN D $4 \mathbf{1 1}$ ist nicht zu erkennen, inwieweit wir diesen Text mit jenen aus Knossos vergleichen dürfen, die Tiere verzeichnen, welche ähnlich zum Palastgeschäft an „Besitzer“ vergeben wurden, oder ob es sich um einen Text handelt, der eher mit der pylischen Cn-Serie in Einklang zu bringen ist. ${ }^{260}$ Wir müssen wohl damit rechnen, dass die Tiere an die Gottheit Hermes beziehungsweise dessen Heiligtum gehen, also vom Palast abgegeben werden. Wenn wir uns allerdings die Texte betrachten, die po-ti-ni-ja-we/-(i-)jo verzeichnen, zeigt sich das Interesse des Palastes an der Wollproduktion der darin aufgeschriebenen Schafe. Haben wir es nun mit einer Vergabe von Tieren an die Göttin Potnia beziehungsweise deren Heiligtum zu tun? Nach ST. HiLleR ist es wahrscheinlich, dass Wolle in tempeleigenen Werkstätten („Tempelwirtschaft“) verarbeitet wurde. ${ }^{261}$ Doch warum hatte dann der Palast Interesse an der Aufzeichnung des Wollertrages der zur knossischen Potnia gehörigen Schafe? ST. HILlER sah die mykenische Tempelwirtschaft als vom zentralen Palastzentrum kontrolliert und in dessen Wirtschaftssystem integriert an. ${ }^{262}$ Dies scheint eine einleuchtende Erklärung, weshalb die Paläste von Pylos und Knossos nicht nur die Abgabe von Tieren an bestimmte Gottheiten verzeichneten sondern, wie im Falle der Potnia der knossischen Dl-Serie auch an der Wollproduktion interessiert waren.

\subsubsection{4. „Hirten“ und Schafe, Ziegen, Schweine in Pylos}

Neben den Aufzeichnungen von Tieren, die entweder zu alt oder zu färbig für die Wollproduktion waren, und jenen, die an „Collectors“ verteilt wurden, finden sich in den folgenden Texten der Cn-Serie auch Schafe, Ziegen und Schweine(!), die ausschließlich mit einem Ortsnamen und einem „Hirten“Namen verzeichnet sind: Cn 45.7.13 lat.inf., Cn 437.2.4, Cn 599.5.7.8, Cn 600.1.2.3.4.9.10.12.13.14.15, Cn 643.2, Cn 655.3.4, Cn 925.1.2.3.

\footnotetext{
257 Olivier 1967, 75, 77ff., 82f.; Olivier 1972, 23ff.; Bennet 1992, 85f. Tab. 5: nach den Da-Dg und Dn-, Dv-Serien zu schließen gingen 29 \% der Schafe an „Besitzer“ und 71 \% an „Hirten“.

${ }^{258}$ Zur Interpretation von po-ti-ni-ja-we-jo s. Aura Jorro 1993, s.v.

259 Vgl. dazu Nosch 2000, 211ff.

260 Zur PY Cn-Serie s. S. 41ff.

${ }^{261}$ Hiller 1982, 97.

${ }^{262}$ Hiller 1982, 99.
} 
PY

Cn 45

.0

.1

pu-ro , rạ-wạ-rạ-ți-jọ[ pa-ro

supra mutila

$\mathrm{S} 4-\mathrm{H} 21$

pu-ro , ra-wa-ra-ti-jo , pa-ro ko-so-ne , we-da-ne-wo ovis ${ }^{t} 80[$

.3 a

\{

pu-ro , ra-wa-ra-ti-jo , pa-ro , e-ko-to-ri-jo , we-da-ne- ovis' 30[

.4

u-po-ra-ki-ri-ja , pa-ro, de-mo-qe, we-da-ne-wo

ovis' $^{\prime} 70$

.5

$.6 j a$

u-po-ra-ki-ri-ja , pa-ro, pe-qe-we , a-ke-o-jo

ovis $^{t} 65$

u-po-ra-ki-ri-ja , pa-ro , do-ro-jo-jo , a-ko-so-ta-

CAP $^{t} 35$

.7

u-po-ra-ki-ri-ja , pa-ro , po-ko-ro

CAP $^{t} 20$

8 a

pu-ro ra-wa-ra-ti-jo , pa-ro , a-ko-to-wo , a-ke-o-

$\mathrm{CAP}^{t} 50$

pu-ro ra-wa-ra-ti-jo, pa-ro , o-qe-qa , a-ko-so-ta-o

CAP $^{t} 40$

10

pa-na-pi , e-ne-ti-jo , a-ko-so-ta-o

u-po-ra-ki-ri-ja , wi-ja-te-we , a-ke-o-jo

CAP $^{t} 3,4$

CAP' $^{\prime} 15$

pu-ro , ra-wa-ra-ti-jo , ka-ra-wa-ni-ta , a-ke-o-jo $\quad$ CAP $^{\mathrm{m}} 100$

.12

po-ma-ko sus ${ }^{i} 16$

po-te-u sus' 7

45 lat.inf.

PY

Cn 437.0

.1

.2

3

.4

5

PY

Cn 599

.1 a
.2
.3
.4
.5
.6
.7
.8

PY

Cn 600

.1
.2
.3
.4
$.5 / a$
.6
.7
.8
.9
.10
.11
.12
.13
.14
.15

qe-ta-ko sus 11

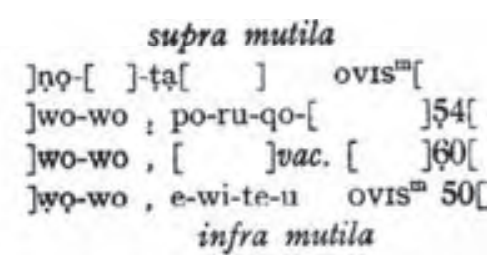

pa-ro

wa-no-jo , wo-wo , ne-ti-ja-no a-ke-o-jo

$a_{2}$-ne-u-te , pa-ro , ka-so , a-ko-so-ta-o

CAP $^{m} 100$

S719- H1

a-pa-tu-wo-te , pa-ro, a-ke-ra-wo, a-ke-o-jo cAP ${ }^{m} \mathbf{9 0}$

$a_{2}$-pa-tu-wo-te , pa-ro , ru-we-ta , a-ke-o-jo CAP $^{t} 40$

$a_{2}$-pa-tu-wo-te, pa-ro , a-wo-i-jo ,

CAP $^{t} 50$

wa-no-jo , wo-wo , pa-ro , ke-re-no , a-ke-o-jo CAP' 80

$a_{2}$-pa-tu-wo-te , pa-ro , e-zo-wo

$\operatorname{sus}^{t} 30$

e-ko-me-no , pa-ro, ti-ri-po-di-ko

$\operatorname{sus}^{t} 57$

$$
\begin{aligned}
& \text { o-re-e-wo, wo-wo, pa-ro , ke-we-no } \\
& \text { o-re-e-wo, wo-wo, de-ko-to } \\
& \text { o-re-e-wo, wo-wo, ke-ro-u-te-u } \\
& \text { o-re-e-wo, wo-wo, e-te-re-ro }
\end{aligned}
$$

$\mathrm{S} 4-\mathrm{H} 21$

- jo

o[-re-e-]wọ , wo-wo , mi-ka-ri-jo , a-ke-o-

re-qa-se-wo, wo-wo, wa-ra-wo-po, A $^{a-k e-p-j o}$

ti-mi-to , a-ke-e, te-[

ti-mi-to, a-ke-e, a-[ ]-u, we-da-ne-wo

re-qa-se-wo, wo-wo, ka-wi-ta

re-qa-se-wo, wo-wo, wi-ja-te-we

ovis $^{\text {tw }} \mathbf{5 0}$

ovis $^{\mathrm{m}} 100$

ovis $^{\text {II }} 90$

ovis $^{\text {m }} 90$

ovis $^{\text {ma }} 92$

ovis $^{\text {tit }} 70$

]pyis 80

ovis $^{t} 60$

ovis $^{t} 70$

ovis $^{t} 80$

ti-mi-to , a-ke-e , o-pe-se-to , a-ke-o-jo ovis ${ }^{t} 60$

ti-mi-to , a-ke-e, e-te-wa-jọ ,

ti-mi-to , a-ke-e, a-no-ze-we

CAP $^{t} 30$

CAP $^{\mathrm{t}} 36$

ti-mi-to , a-ke-e , qe-ta-ko

sus $^{\text {min }} 30$

ti-mi-to , a-ke-e , ${ }^{*} 82$-de[ ]

sus $^{1} 12$

reliqua pars sine regulis 
PY

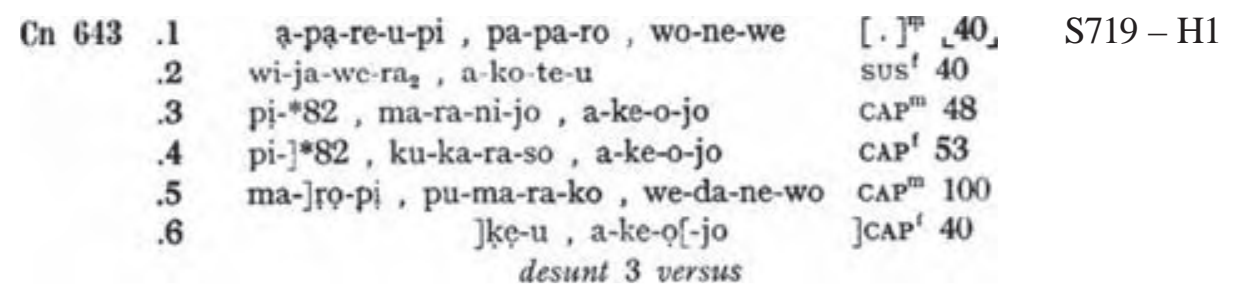

PY

S4 - H21

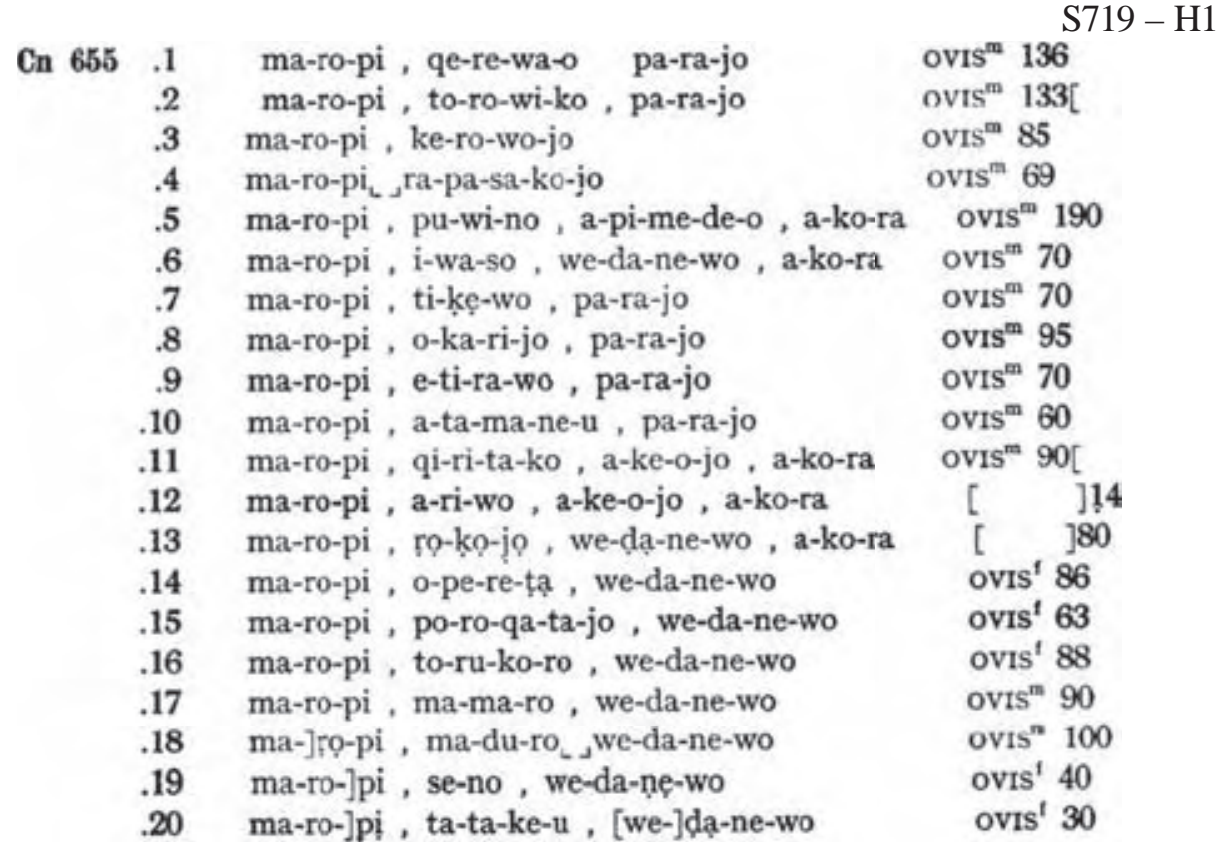

PY

Cn 925 .
.2
da-we-u-pi , pa-ro , ko-ma-we-te $\operatorname{sus}^{m} 16$
S925 - H1 $\begin{array}{ll}\text { da-we-u-pi , pa-ro , e-te-we } & \text { sus }^{t} 16 \\ \text { da-we-u-pi , pa-ro , e-do-mo-ne-we } & \operatorname{sus}^{t} 28\end{array}$

Für die Aufzeichnung dieser „Hirten“-Namen gibt es kein einheitliches Muster. Sie wurden mit der Präposition pa-ro verbunden und stehen im Dativ (Cn 45.7 pa-ro po-ko-ro, Cn 599.5 pa-ro a-wo-i-jo, Cn 599.7 pa-ro e-zo-wo, Cn 599.8 pa-ro ti-ri-po-di-ko, Cn 600.1 pa-ro ke-wo-no, Cn 925.1 pa-ro ko-ma-we-te, Cn 925.2 pa-ro e-te-we, Cn 925.3 pa-ro e-do-mo-ne-we), sind ohne pa-ro im Nominativ (Cn 45.13 po-ma-ko, Cn 45.13 po-te-u, Cn 45 lat.inf. qe-ta-ko, Cn 600.2 de-ko-to, Cn 600.3 ke-ro-u-te-u, Cn 600.4 e-te-re-ro, Cn 600.9 ka-wita, Cn 600.10 wi-ja-te-we, Cn 600.12 e-te-wa-jo, Cn 600.13 a-no-ze-we, Cn 600.14 e-wi-te-u, Cn 643.2 a-ko-te-u) oder im Genetiv (Cn 655.3 ke-ro-wojo, Cn 655.4 ra-pa-sa-ko-jo) vorhanden. Die möglichen Übersetzungen lauten also „von NN“, „,bei NN“, „des NN“ und „der NN“. Wodurch mag diese uneinheitliche Schreibweise der „Hirten“-Namen verursacht sein? Sie ist umso auffälliger als die Namen der „Collectors“, bis auf wenige Ausnahmen, immer im Genetiv erfasst wurden. Könnte es sich bei den nur mit „Hirten“-Namen verzeichneten Schafen, Ziegen und Schweine um Tiere gehandelt haben, die vom pylischen Palast den „Hirten“ als deren Besitz überlassen wurden? Im System des orientalischen Palastgeschäftes war es möglich, dass ein Hirte Tiere, die er als „Überschuss“ erwirtschaftete, seinem eigenen Herdenbestand einverleib- 
te. ${ }^{263}$ Wenn wir annehmen, dass jene Schafe, Ziegen und Schweine der oben angeführten Texte von den pylischen „Hirten“ über das vom Palast verlangte Soll gezüchtet und ihnen dafür überantwortet wurden, ließe sich auch die Konfusion der Namensschreibungen erklären: die Tiere waren demnach bei den „Hirten“, von den „Hirten“ und für die „Hirten“. Diese Mehrdeutigkeit schlug sich offensichtlich auch in der schriftlichen Abfassung der Bestandsaufnahme nieder. ${ }^{264}$

Ein solcher „Hirte“ aus Pylos war vermutlich ke-ro-wo, dessen Name sich auf den Tafeln PY Ae 134, Cn 4.9, Cn 131.7 und Cn 655.3 findet. ${ }^{265}$ Dieser kero-wo wird auf Ae 134 als po-me („Hirte“) bezeichnet und war für „Vierfüßler“ des „Besitzers“ ta-ra-ma-ta verantwortlich. Der Name ke-ro-wo(-jo)/ke-ro-we findet sich auch auf den Tafeln PY Cn 131.7 und Cn 655.3 als „Hirte“ von Schafen in ma-ro-pi sowie auf PY Cn 4.9 mit fehlenden Schafen (OVIS + TA) in der Stallung (ta-to-mo) von a-si-ja-ti-ja verzeichnet. Nachdem er demzufolge für Tierherden des Palastes (Cn-Serie) verantwortlich war und nach Ae 134 einen Mann zur Bewachung der Herden des Thalamātās abgestellt hatte, dürfen wir in ke-ro-wo wohl einen „Hirten“ analog zu jenen der knossischen D-Serien sehen. Doch ist für ihn kein Nachweis zu erbringen, dass er auch außerhalb der Schafhaltung Tätigkeiten ausübte.

Auf PY Ae 264 ist ein $a_{3}$-ki-pa-ta (,Ziegenhirte“) namens pi-ra-jo verzeichnet. Derselbe Name findet sich auch auf der Tafel PY Un 249, hier als a-re-pazo[-o („Salbenkoch“) bezeichnet. Könnte es sich hier um dieselbe Person handeln, die je nach der Erfassung ihrer Tätigkeit als Ziegenhirte oder Salbenkoch bezeichnet wurde? Oder sollte es sich bei pi-ra-jo um einen Salbenkoch handeln, der auch die Verantwortung über palatiale Ziegenherden übernahm? ${ }^{266}$ Obwohl pi-ra-jo als Ziegenhirte bezeichnet wurde, findet sich sein Name jedoch nicht in der pylischen $\mathbf{C n}$-Serie, weshalb kein sicherer Hinweis vorhanden ist, dass er auch die Verantwortung über palasteigene Herden innehatte.

Doch sind in der pylischen Cn-Serie ebenfalls Namen verzeichnet, die sich auch in anderen Serien finden (Tab. 12). Sicher müssen bei gleichlautenden Namen nicht dieselben Personen angenommen werden. Das Vorkommen derselben Personennamen auf Texten, die Schafe, Ziegen sowie Schweine verzeichnen, und auf solchen anderer Tätigkeitsfelder, kann jedoch nicht als pure Namensgleichheit abgetan werden. ${ }^{267}$

Von den 49 Namen, die nicht nur in der PY Cn-Serie vorkommen, finden sich insgesamt 23 in der pylischen Jn-Serie. ${ }^{268}$ Diese befasst sich mit Zuteilungen von Bronze an verschiedene Schmiede ( $k a-k e-w e)$.

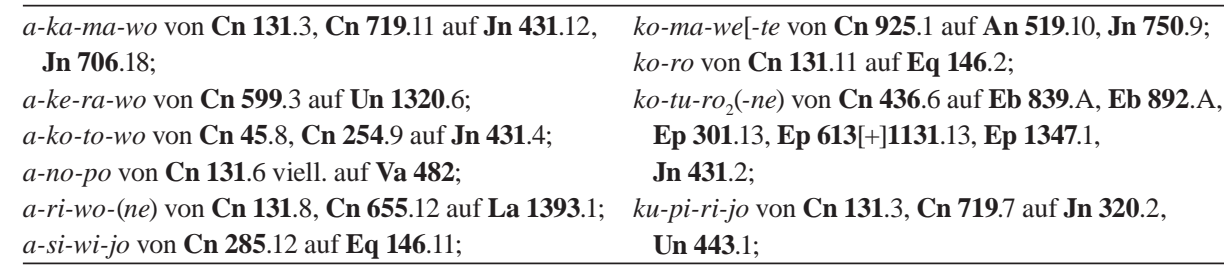

263 Renger 2000, 164.

264 Über die Auswirkungen auf die zu vermutende Gesamtzahl der pylischen Schafe s. S. 73ff. und Fn. 294.

265 Siehe auch S. 66ff.

${ }^{266}$ Vgl. dazu die „Hirten“, welche nach der pylischen Jn-Serie Schmiede waren, s. S. $64 f$.

267 Siehe zum Thema der Namensgleichheit und der möglichen Bestimmung als dieselben Personen Deger-Jalkotzy 2001, 69; s. ferner Lindgren 1973 I, 10; Lang 1988, $190 \mathrm{ff}$.

${ }^{268}$ Vgl. Deger-Jalkotzy 2001, 70ff. 


\section{An 661.3;}

ka-do-wo von Cn 719.1 auf Aq 64.3;

ka-ra-u-ko von Cn 285.4 auf Jn 706.8, Jn 832.5;

$k a$-ta-wa von Cn 40.13 auf Jn 605.4;

$k e-r o-u-t e[-u$ von Cn 600.3 auf Fn 324.16;

ke-sa-me-no von Cn 131.13 auf Fn 324v.1;

ke-we-no von Cn 600.1 auf Jn 431.23;

Tab. 12: Die Namen der „Hirten“ aus der pylischen Cn-Serie und ihr Vorkommen in weiteren Texten.

Wenn es sich bei den „Hirten“ und den Schmieden um dieselben Personen handelte, könnte dies heißen, dass einzelne Schmiede gleichzeitig die Verantwortung über palatiale Herden übernahmen. War einer darunter vielleicht sogar ein te-o-jo do-e-ro („Gottesdiener“) mit Namen e-do-me-ne-u, der somit eine kultische Funktion hatte? C. GILLIS hielt es für möglich, dass die Schmiede zeitweise als Metallhandwerker arbeiteten und zeitweise als Hirten tätig waren. ${ }^{269}$ Vielleicht sollten wir jedoch davon ausgehen, dass einzelne Schmiede zusätzlich zu ihrem Beruf die Verantwortung über palatiale Herden übernommen hatten. ${ }^{270}$ Wenn wir von der Interpretation C. GiLLIS ausgehen, dass „the smith was not a palace employee“, könnte die Vergabe von Palastherden an verschiedene Schmiede eine Möglichkeit gewesen sein, diese an den Palast zu binden.

Die Bindung an das zentralistische Palastzentrum durch für den Palast ausgeführte verschiedene Arbeiten wurde von S. DEgER-JALKOTZY in ihrem Artikel Working for the Palace: PY An 261 vorgestellt. ${ }^{271}$ „These conditions must have led to a considerable mobility of the general working force and consequently may well have formed the background of prosopographical overlappings between texts relating to different occupations, as well as to a variety of place-names“. ${ }^{272}$ Die Schmiede selbst könnten sich durch die Tierhaltung jedoch auch ein zusätzliches „Einkommen“ verschafft haben. So konnten sie sich wahrscheinlich einen Überschuss an gezüchteten Tieren behalten und erhielten möglicherweise dunkles, nicht für die Wollproduktion geeignetes Vieh. ${ }^{273}$

269 Gillis 1997, 512.

270 Vgl. Deger-Jalkotzy 2001, 70.

${ }^{271}$ Deger-Jalkotzy 2001, 70.

272 Deger-Jalkotzy 2001, 69f.

273 Siehe dazu S. $48 f$ u. Fn. 212; Orth 1921, 388; Kroll 1924, 596; Postgate 1992, 160. 
Eines ist ebenfalls festzuhalten, auch die „Hirten“ der pylischen Cn-Serie gehörten offensichtlich der gesellschaftlichen Mittelschicht an, wenn man bedenkt, dass sie in verschiedenen Wirtschaftssektoren, zum Beispiel dem Schmiedehandwerk tätig waren, aber auch zur Ablieferung landwirtschaftlicher Produkte (PY Ma- und Na-Serie) an den Palast verpflichtet waren. ${ }^{274}$ Ein weiteres Indiz für die gehobene Stellung liefert der Name i-ma-di-jo (Cn 436.5), dessen Träger nach PY Ea 29 und Ea 827 sogar in der Lage war, Grundbesitz an andere Personen zu vergeben, wie dies auch die als po-me, su-qo-ta und qo-qo-ta bezeichneten Männer derselben Serie konnten. Könnte es sich bei diesem i-ma-di-jo vielleicht sogar um einen mit diesen vergleichbaren „Besitzer" gehandelt haben? Aus den knossischen D-Serien wissen wir, dass manche der „Besitzer“ auch zur Kategorie der „Hirten“ gehörten. ${ }^{275}$ Doch lässt sich i-ma-di-jo aufgrund seiner Eintragung auf Ea 29 und Ea 827 nicht eindeutig als „Besitzer“ festlegen, ${ }^{276}$ da wir damit rechnen müssen, dass auch ein „Hirte“ Grundbesitz haben konnte.

Ein weiteres Indiz für die Vergabe der Tiere an die „Hirten“ bieten die Gründe für die Textaufzeichnungen selbst. Alle bis jetzt besprochenen Schafe und Ziegen (pa-ra-jo, wo-ne-we, „Collector“-Besitz) standen dem pylischen Palast nicht für die Wollproduktion zur Verfügung. Wenn wir die in den noch zu besprechenden we-re-ke-Texten erfassten Schafe und Ziegen beiseite lassen, würde das Verhältnis von „Collector“-Herden und Palastherden für letzteren durchaus mager ausfallen. Aber warum sollten in denselben Texten einige wenige Schafe, Ziegen, aber auch Schweine registiert worden sein, die für die palatialen Herden bestimmt waren? Wäre es nicht sinnvoller gewesen dafür eigene Aufzeichnungen/Tafeln anzufertigen? Vielleicht sollten wir tatsächlich davon ausgehen, dass das ausschließlich mit „Hirten“-Namen versehene Vieh vom Palast als Überschuss an die „Hirten“ abgegeben wurde und die Schafe somit dem Palast ebenfalls nicht als Wolllieferanten dienten. ${ }^{277}$

Über die unterste Stufe von Beschäftigten, die mit den orientalischen „Unterhirten“ vergleichbaren Männer, erfahren wir aus den Linear B-Texten der knossischen D-Serien nichts. ${ }^{278}$ Die Palastverwaltung verzeichnete nur die für sie relevanteren Personen, die dem Palast gegenüber verantwortlich waren. Doch scheint es mir in den pylischen Aufzeichnungen Hinweise auf solche „Unterhirten“ zu geben. Diese finden sich nicht in der PY Cn-Serie sondern in der PY Ae-Serie. Zu nennen sind dabei die Texte der Tafeln PY Ae 27, Ae 108, Ae 134 und Ae 489.

274 Deger-Jalkotzy 2001, 70.

275 Siehe S. 55.

276 Siehe S. 67.

277 Bislang wurden alle Tiere ohne „Besitzer“ als Palastbesitz gedeutet, s. L. R. Palmer 1957, 570; Olivier 1967, 79; Olivier 1972, 23f., 27.

278 Auch in den mesopotamischen Texten finden sich keine Namen der untersten Schicht der Hirten; s. dazu van de Mieroop 1992, 86; van Driel 1993, 220: „The documentation reflects, as always, the interest of the owners, or their top-level managers“; Postgate 1992, 160. 
Verzeichnet sind in diesen Texten jeweils drei Personen. Betrachten wir dies am Beispiel Ae 134. Zunächst wird ein Mann namens ke-ro-wo angeführt, der als po-me („Hirte“) bezeichnet wird. Derselbe Name findet sich auf der Tafel PY Cn 131.7 als „Hirte“ von Schafen in ma-ro-pi und auf PY Cn 4.9 mit fehlenden Schafen (OvIS + TA) in der Stallung (ta-to-mo) von a-si-ja-ti-ja. Wir können ke-ro-wo als „Hirten“ analog zu jenen der knossischen D-Serien betrachten, da ke-ro-wo und seine Kollegen der pylischen Cn-Serie offensichtlich für die palatialen Herden verantwortlich waren. ${ }^{279}$ Außerdem scheint sein Name im Genetiv ke-ro-wo-jo auf PY Cn 655.3 auf. Dies ist eine der wenigen Eintragungen der pylischen $\mathbf{C n}$-Serie (außer den we-re-ke-Texten) in der auf den Hirtennamen weder die Wörter pa-ra-jo beziehungsweise wo-ne-we noch der Name eines „Collector“ folgt. Könnte es sich bei Cn 655.3 um Tiere handeln, die ke-ro-wo aus einem erwirtschafteten Überschuss der im anvertrauten Schafe erhielt? Dies ist jedenfalls im orientalischen Palastgeschäft durchaus möglich. 280

Auf PY Ae 134 folgt auf die Nennung des Hirten das Toponym a-si-ja-ti-ja. Wenn wir die Eintragungen der KN D-Serien betrachten, ist dort der Ortsname in den Texten an gleicher Stelle geschrieben. Es ist folglich anzunehmen, dass sich a-si-ja-ti-ja auf die ebenfalls in Ae 134 genannten qe-to-ro-po-pi (,Vierfüßler") bezieht, die sich an diesem Ort befanden beziehungsweise administrativ dorthin gehörten. ${ }^{281} \mathrm{Im}$ Anschluss an den Ortsnamen folgen die Worte $o$-pi qe-to-ro-po-pi ta-ra-ma-ta-o. Diese Angabe bezieht sich auf die im Folgenden genannten „Vierfüßler“ und lautet somit „über die Vierfüßler des ta-ra-ma-ta“. Zum Schluss des Textes folgen die Eintragungen VIR 1 und o-ro-me-no. Das Wort o-ro-me-no wird mit dem klassisch-griechischen öpo $\mu \alpha 1$ („,bewachen“) ${ }^{282}$ verbunden. Der mit VIR 1 erfasste Mann wird wohl derjenige gewesen sein, der für die Bewachung, also das Hüten der Tiere zuständig war. Ich denke nicht, dass es sich hierbei um den zu Beginn genannten ke-ro-wo gehandelt haben wird. In diesem Falle hätte die bloße Angabe ke-ro-wo po-me ausgereicht. Vielmehr scheint es so, dass die Tafel PY Ae 134 die Aufzeichnung darüber enthält, dass ,vom Hirten ke-ro-wo in a-si-ja-ti-ja über die Vierfüßler des tara-ma-ta ein Mann [,Unterhirte“] wachend“ abgestellt beziehungsweise angestellt wurde. Das Interesse des Palastes an der Erfassung der „Unterhirten“ in der Ae-Serie dürfte wohl damit begründet sein, dass die Palastverwaltung über alle Tätigkeiten seiner Untergebenen - und wahrscheinlich auch im Dienste des Palastes stehenden Personen - informiert sein wollte.

Wie bereits erwähnt findet sich der Name ke-ro-wo auch in der pylischen Cn-Serie (Cn 131.7, Cn 4.9; Cn 655.3). Nachdem er den Texten der Cn-Serie zufolge für Tierherden des Palastes verantwortlich war und nach Ae 134 einen Mann zur Bewachung der Herden des Thalamātās abgestellt/angestellt hatte,

\footnotetext{
279 Siehe S. 59ff.

280 Renger 2000, 164.

281 Siehe dazu S. 68ff.

282 Bartoněk 2003, 320.
} 
dürfen wir in ke-ro-wo wohl einen „Hirten“ analog zu jenen der knossischen D-Serien sehen. Dementsprechend müsste es sich bei ta-ra-ma-ta um einen „Besitzer“ („Oberhirten“) gehandelt haben. Dieser ta-ra-ma-ta hatte nicht nur verschiedenste Herden an „Hirten“ vergeben, die Männer zur Bewachung abstellten (PY Ae 108, Ae 134, Ae 489) sondern nach Ausweis der pylischen Ea-Serie Landbesitz der unterschiedlichsten Kategorien ${ }^{283}$ : Ea 336 ko[-to-na unbekannter Form, Ea 821 ko-to-na ki-ti-me-na, Ea 825 o-na-to pa-ro ko-do po-me-ne, Ea 778 o-na-to pa-ro da-mo. In derselben Serie finden sich auch Personen, die als su-qo-ta-o (PY Ea 59.3, Ea 109, Ea 132, Ea 480, Ea 481.2, Ea 776, Ea 822), qo-qo-ta-o (PY Ea 270, Ea 305, Ea 757, Ea 802) und po-me (-ne) (PY Ea 71, Ea 439, Ea 782, Ea 800, Ea 824, Ea 825, Ea 817) verzeichnet sind. Darunter sind zumindest folgende namentlich belegt: ein pe-re-qo-no qoqo-ta (Ea 270), ein mo-ro-qo-ro po-me (Ea 439, Ea 782, Ea 800, Ea 817) und ein ko-do po-me-ne (Ea 71, Ea 824, Ea 825); die Eintragungen von qo-qo-ta und su-qo-ta ohne Personennamen könnten sich auf jeweils ein und dieselben Personen beziehen oder aber auf mehrere, deren Namen nicht genannt wurden. Doch warum wurden sie als „(Schaf)-Hirte“ (po-me/-ne), „Sauhirte“ (su-qo-ta) und „Rinderhirte“ (qo-qo-ta) bezeichnet, obwohl sie in keinem Text zu finden sind, der sich mit der Tierhaltung beschäftigt? Ich denke, dass sie trotzdem als „Besitzer“ („Oberhirten“) zu sehen sind, und wohl in Zusammenhang mit ihren Verantwortungsbereichen als Schaf-, Rinder- und Schweinehirten bezeichnet wurden. ${ }^{284}$ Weiters lässt sich vermutlich auch ta-ra-ma-ta in diese Gruppe von Männern einordnen. Er wird in der Ea-Serie zwar nicht als po-me oder dergleichen genannt, doch hat er nach Ausweis der Ae-Serie eindeutig Viehherden in seinem Besitz. Dass er gleichzeitig bei ko-do, einem seiner Kollegen, Landbesitz hatte, stellt dabei kein Problem dar. Betrachten wir nur die vielfältigen wirtschaftlichen Belange, in die die „Besitzer“ der knossischen D-Serien involviert waren. ${ }^{285}$

Damit lassen sich die Personen aus Pylos, welche als po-me, su-qo-ta und qo-qo-ta bezeichnet wurden und ta-ra-ma-ta mit den knossischen „Besitzern“ der $\mathbf{D}$-Serien vergleichen, nicht jedoch mit den „Collectors“ der pylischen $\mathbf{C n}$ Serie. Weshalb die pylischen „Besitzer“ („Oberhirten“) nicht mit Tierherden verzeichnet sind, die ähnlich zu jenen der knossischen D-Serien erfasst wurden, liegt entweder an unterschiedlichen Zeitpunkten der Aufzeichnungen, oder es sind uns aus Pylos und Knossos einfach andere Serien erhalten geblieben.

Als Schlussbetrachtung ließe sich sagen, dass die mykenischen Paläste in der Herdenhaltung durchaus ein System betrieben haben könnten, das vergleichbar zu den Palastgeschäften des Alten Orients war. Dennoch kann nicht festgestellt werden, ob es sich um absolut dasselbe System gehandelt hat. Gleichzeitig wäre es möglich, dass es sich bei den „Besitzern“ der knossischen D-Serien um Personen handelte, die mit ta-ra-ma-ta der pylischen Ae-Serie und den als su-qo-ta, qo-qo-ta und po-me/-ne bezeichneten Männern der PY Ea-Serie vergleichbar sind. Die „Besitzer“ der knossischen D-Serien und der pylischen Ae-Serie unterscheiden sich von den „Collectors“ der PY Cn-Serie dadurch, dass sie die Verwaltung palatialer Herden übernahmen, während die

${ }^{283}$ Zur Interpretation des ta-ra-ma-ta auf den Texten der PY Ae-Serie und der PY Ea-Serie s. Lindgren 1973 I, 114.

${ }^{284}$ Vgl. dazu Deger-Jalkotzy 1988, 119.

${ }^{285}$ Vgl. dazu den knossischen „Besitzer“ ka-to-ro, der ebenfalls eine Schafherde eines anderen „Besitzers“ übernahm; s. S. 55. 
„Collectors“ die Herden vom Palast als Versorgungsgüter erhielten. ${ }^{286}$ Es ist an dieser Stelle ausdrücklich festzuhalten, dass der Unterschied zwischen den Personen - den „Collectors“ in den pylischen und den „Besitzern“ in den knossischen Texten - auf der rein formalen Unterscheidung der Textinhalte beruht. Auf der einen Seite handelt es sich bei der PY Cn-Serie um Aufzeichnungen über die Abgabe verschiedener Tiere unter anderem als Versorgungsgüter an vier Männer der höheren pylischen Gesellschaftsschicht. Dies sagt nichts über etwaige Aktivitäten der als „Collectors“ definierten Personen aus. Zum anderen verzeichnen die knossischen Texte die Vergabe von Tieren (Schafen) an unterschiedliche Männer, wobei die Verantwortung über die Haltung der palatialen Herden in deren Hände gelegt wird. In diesen Fällen wird das Tätigkeitsfeld dieser Personen - die Schafhaltung - ersichtlich. Ob we-u-da-ne-u, $a-k o-s o-t a, a-k e-o$ und a-pi-me-de ebenfalls Tierherden des pylischen Palastes in Form des Palastgeschäftes übernahmen, lässt sich aufgrund der nicht vorhandenen Texte, welche mit den knossischen $\mathbf{D}$-Serien vergleichbar wären, nicht sagen. Des Weiteren kann auch für die „Besitzer“ der KN D-Serien und der PY Ae-Serie keinesfalls ausgeschlossen werden, dass sie von den jeweiligen Palastzentren ebenfalls mit Tieren als „Versorgungsgüter“ ausgestattet wurden.

\subsubsection{PY Cn 4 und Cn 595}

PY

\begin{tabular}{|c|c|c|c|}
\hline Cn 4 & $\begin{array}{l}.1 \\
.2 \\
.3 \\
.4 \\
.5 \\
.6 \\
.7 \\
.8 \\
.9 \\
.10 \\
.11\end{array}$ & 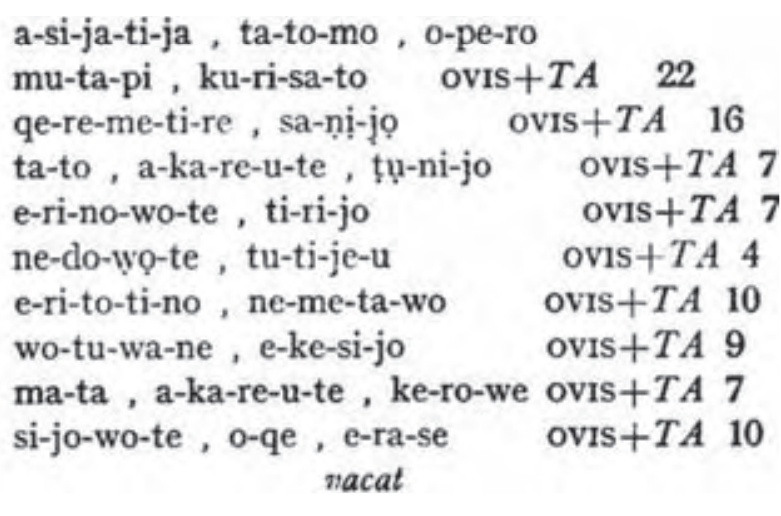 & $\mathrm{S} 4-\mathrm{H} 21$ \\
\hline & & & \\
\hline Cn 595 & $\begin{array}{l}.1 \\
.2 \\
.3 \\
.4 \\
.5 \\
.6 \\
.7 \\
.8\end{array}$ & 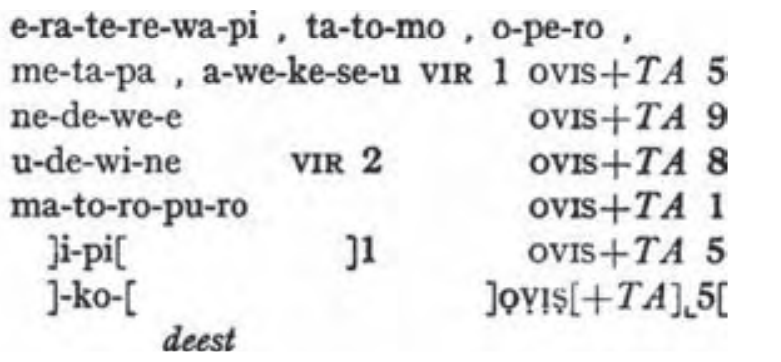 & $\begin{array}{r}\mathrm{S} 4-\mathrm{H} 21 \\
\mathrm{H} 1\end{array}$ \\
\hline
\end{tabular}

Auf die Interpretation der Angabe ovIs + TA als Definition für männliche Zuchttiere wurde bereits im Abschnitt Das Ideogramm ovIS + TA eingegangen. ${ }^{287}$ Die Schafe der Texte Cn 4 und Cn 595 sind als fehlende (o-pe-ro) Tiere

${ }^{286}$ Damit sollten die knossischen „Besitzer“ nicht als „Collectors“ definiert werden. Bereits Godart 1992, 362 wies darauf hin, dass der Ausdruck a-ko-ra der pylischen Cn-Serie nicht auf alle Namen im Genetiv zu übertragen ist.

${ }^{287}$ Siehe S. 36ff. 
in den Stallungen (ta-to-mo) der beiden Ortschaften a-si-ja-ti-ja und e-ra-tere-wa registriert. Es handelte sich also um Widder, die nicht für die Zucht zur Verfügung standen. Sie wurden anscheinend von den in den Texten ebenfalls angeführten Personen und Gemeinden nicht zu den Zuchtstationen gesandt. ${ }^{288}$ Während die Tafel $\mathbf{C n} \mathbf{4}$ verschiedene für die nicht erfolgte Lieferung verantwortliche Personen aus unterschiedlichen Ortschaften auflistet, ist auf Cn 595 nur in der Zeile .2 ein Personenname ( $a$-we-ke-se- $u$ ) erfasst. Die weiteren Zeilen nennen, soweit ersichtlich, ausschließlich Ortsnamen. Auffällig ist allerdings die Angabe der Anzahl von Männern ohne Namen - vIR 1 in Zeile .2, vIR 2 in Zeile .4 und vermutlich VIR 1 in Zeile .6. Wäre es nicht möglich, dass aufgrund der Nennung von $a$-we-ke-se-u gleich zu Beginn der Auflistung, dieser als „Hirte“ für die fehlenden Tierlieferungen aus den unterschiedlichen Orten verantwortlich war? Aus den weiteren Texten der pylischen Cn-Serie wissen wir, dass ein „Hirte“ durchaus für Tierherden in verschiedenen Ortschaften zuständig gewesen sein konnte. Könnten die zumindest für drei Orte ebenfalls angeführten Männer (VIR) nicht als „Unterhirten“ analog zur PY Ae-Serie gesehen werden? Diese wären dann die dem $a$-we-ke-se-u unterstellten tatsächlichen Hirten gewesen. Doch verantwortlich gegenüber dem Palast für die nicht erfolgten Lieferungen von Schafen war a-we-ke-se-u.

Zusammenfassend lässt sich sagen, dass alle bis jetzt besprochenen Texte der Cn-Serie Tiere verzeichnen, welche der pylische Palast der vorhergehenden Interpretation zufolge anscheinend nicht zur Wollproduktion einsetzen konnte.

2.1.3. we-re-ke, Schafe und Ziegen

PY

\section{Cn 131}

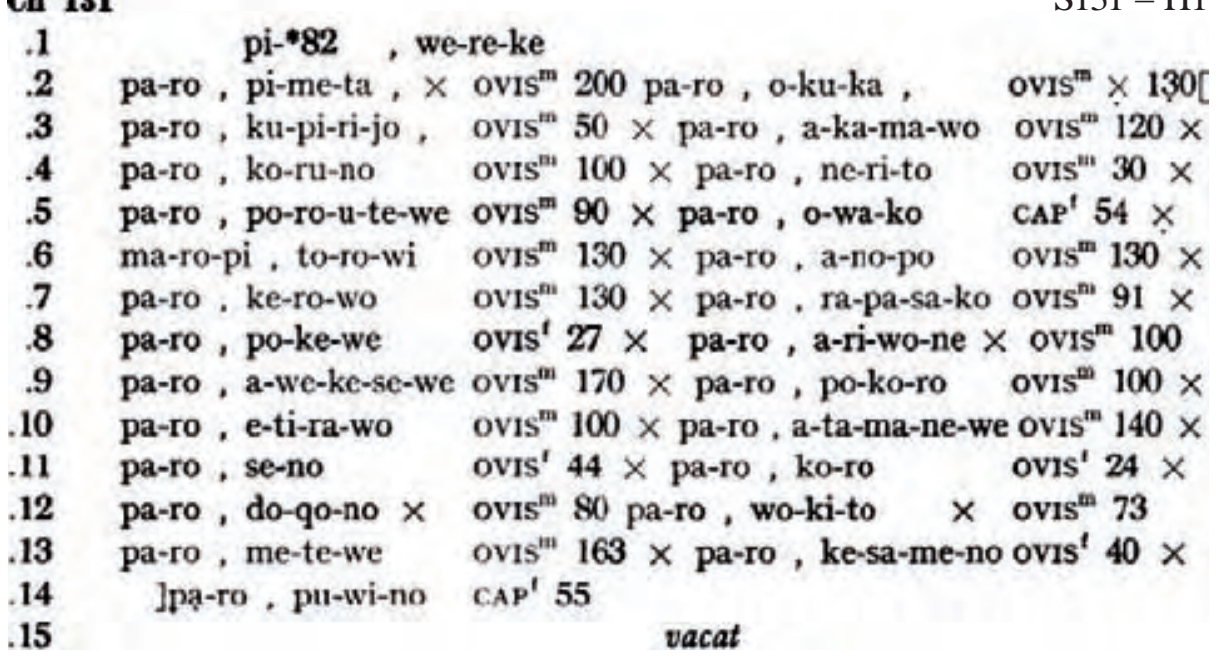

PY

Cn 202

.1
.2
.3
.4
.5

]a-ke-re-wa , we-re-ke

]me-wi-jo , o-ki-ri-so ovis 80[

Jka-sa ovis ${ }^{\mathrm{m}} 102$

] ovIS $^{\text {m }} 74$ CAP $^{\mathrm{m}} 1[$

]4

infra mutila

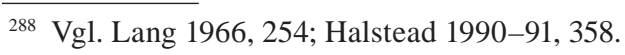


PY

\begin{tabular}{|c|c|c|c|}
\hline Cn 285 & .1 & ro-u-so $\quad[$ & \\
\hline & .2 & $a_{3}$-ta-ro-we & [ \\
\hline & .3 & re-ta-mo & $\mathrm{CAP}^{\mathrm{m}}[$ \\
\hline & .4 & ka-ra-u-ko & CAP'P [ \\
\hline & .5 & a-we-ke-se-u & ovis $^{\mathrm{m}} \mathbf{5 0}$ \\
\hline & .6 & a-we-ke-se-u & $\mathrm{CAP}^{\mathrm{m}} 30[$ \\
\hline & .7 & wa-da-ko & $\mathrm{CAP}^{\mathrm{m}} 86$ \\
\hline & .8 & si-no-u-ro & $\mathrm{CAP}^{\mathrm{tm}} 60[$ \\
\hline & .9 & ra-ma-jo & $\operatorname{CAP}^{t} 20[1$ \\
\hline & .10 & pa-wa-wo & {$[\cdot]^{\prime}[$} \\
\hline & .11 & e-ke-da-mo & ovis $^{\mathrm{m}} 100$ \\
\hline & .12 & a-si-wi-jo & ovis $^{\mathrm{m}} 100$ \\
\hline & .13 & o-ki-ra & ovis $^{\mathrm{m}} 116$ \\
\hline & .14 & o-ti-na-wo & ovis $^{\text {m }} 100$ \\
\hline
\end{tabular}

PY

\begin{tabular}{|c|c|c|c|c|}
\hline \multirow[t]{16}{*}{ Cn 328} & .1 & ro-u-so, & we-re-ke , & S131 - H1 \\
\hline & .2 & a-ka-na-jo, & ma-ro ovis $200 \times$ & \\
\hline & .3 & a-ka-na-jo , & ko-wa-to $\mathrm{CAP}^{\mathrm{m}} 50 \times$ & \\
\hline & .4 & a-ka-na-jo, & ra-mi-ni-jo $\mathrm{CAP}^{1} 40 \times$ & \\
\hline & .5 & a & ma-ra-te-u ovis ${ }^{t} 10 \times$ & \\
\hline & .6 & & da-to-re-u CAP $^{m} 30$ & \\
\hline & .7 & & wo-ki-ro $\operatorname{CAP}^{i} 20 \times$ & \\
\hline & .8 & & ovis $^{t} 50 \times$ & \\
\hline & .9 & & CAP 40 & \\
\hline & .10 & & ovis $^{\mathrm{m}} 40 \times$ & \\
\hline & .11 & & ovis $^{\mathrm{m}} 170 \times$ & \\
\hline & .12 & & wi-sa[ ]ovis $^{t} 60 \times$ & \\
\hline & .13 & & a[ ] ] & \\
\hline & .14 & & pu-za-ko $\quad$ CAP $^{m} \times 40$ & \\
\hline & .15 & & $a_{3}$-ta-ro-we ovis ${ }^{t} 70 \times$ & \\
\hline & .16 & & vacat & \\
\hline
\end{tabular}

Betrachten wir die letzte Kategorie von Texten der PY Cn-Serie, die we-reke-Tafeln. Auf diesen Tafeln sind verschiedene Herden von Schafen und Ziegen (keine Schweine) mit den für sie verantwortlichen „Hirten“ verzeichnet. Zum Unterschied zur vorhergehenden Kategorie der Cn-Texte ist der Ortsname für alle zu einer Lokalität gehörigen Herden und „Hirten“ jeweils nur einmal angegeben. ${ }^{289}$ Auffällig an den we-re-ke-Texten ist zusätzlich die Kennzeichnung einiger Herden mit den sogenannten „Checkmarks“, „Verifikationszeichen in der Form eines Kreuzes, durch das die Registrierungsposten zusammengezählt, verifiziert, bzw. kontrolliert wurden“. ${ }^{290}$ Warum sind auf diesen Tafeln die Schaf- und Ziegenherden als kontrolliert abgehakt worden? Vielleicht gibt uns das Wort we-re-ke die Antwort auf diese Frage. Dieser Begriff wird als „Schafhürde“, „Ställe“, „Pferche“ interpretiert. ${ }^{291}$ Wenn die Tiere, die sich in den Pferchen befanden, verzeichnet sind und deren Anwesenheit zusätzlich nochmals kontrolliert wurde, muss es sich um Tiere handeln, die sich

\footnotetext{
${ }^{289}$ Eine Ausnahme bildet die Tafel PY Cn 436, auf der kein Ortsname und zu finden ist und auch die Angabe we-re-ke fehlt. Aufgrund der Schreiberhand S 131 - H 1 und den „Checkmarks" dürfte sie jedoch ebenfalls zu den we-re-ke-Texten gehören.

290 Bartoněk 2003, 97.

${ }^{291}$ Halstead 1990-91, 354; L. R. Palmer 1963, 168; Ventris-Chadwick 1973, 433; Bartoněk 2003, 232, 381 (vgl. $F \varepsilon \rho \gamma$-,einschließen“, F́́ $\rho \gamma \nu \rho \alpha$ „Hof, Gehege“); Ilievski 1968, $626 \mathrm{f}$.
} 
aus einem besonderen Grunde dort aufhielten. Wenn die Schafe, Ziegen und Schweine der sonstigen Texte der pylischen Cn-Serie als „Abgang“ verbucht wurden, muss es dementsprechend auch Tafeln gegeben haben auf denen „Zugänge“ festgehalten waren. Diese wiederum scheinen die we-re-ke-Texte zu verzeichnen. So sind auf ihnen mit überwiegender Mehrheit Schafe angeführt, von denen der Großteil wiederum männliche kastrierte Tiere sind. Eine solche Zusammenstellung - vorwiegend Hammel - passt ausschließlich auf Tiere, die der Wollproduktion zugeführt werden sollten.

Bei den Ortsnamen, die auf den we-re-ke-Texten zu erkennen sind, handelt es sich durchwegs um solche der größeren Bezirke der Diesseitigen Provinz: pi-*82 (Cn 131.1.), a-ke-re-wa (Cn 202.1), ro-u-so (Cn 285.1). Wir dürfen wohl annehmen, dass die Schafe und Ziegen, welche Wolle liefern sollten, in den Pferchen dieser Bezirke registriert wurden. Daneben scheint es noch „Abteilungen“ in Orten gegeben zu haben, die in den Einzugsbereich solcher Bezirke fielen - in ma-ro(-pi) (Cn 131.6) bei pi-*82 und in ro-u-so a-ka-na-jo (Cn 328.1), welches wohl bei ro- $u$-so anzusetzen ist. Die oben aufgelisteten Gemeinden werden jedoch nicht die einzigen gewesen sein, an denen man Tiere zusammengezogen hat. Ich vermute, dass es entsprechende we-re-ke-Aufzeichnungen für jeden Bezirk/Ort der Diesseitigen und der Jenseitigen Provinz gegeben haben muss. So findet sich auf der Tafel PY Cn 595 der Bezirk e-rate-re-wa (Jenseitige Provinz) verzeichnet, an dem sich eine Stallung (ta-to-mo) befunden hatte. Dies entspricht der Angabe von a-si-ja-ti-ja ta-to-mo auf PY Cn 4 (ebenfalls Jenseitige Provinz). Sollten wir nicht annehmen, dass jeder Bezirk sowohl Tiere registrierte, die für die Wollproduktion gedacht waren (we-re-ke-Texte), und dass sie ebenfalls Stallungen (ta-to-mo) hatten, in denen die männlichen Tiere für die Zucht (OVIS $+T A$ ) gehalten wurden? Dies würde zu den Aufzeichnungen von PY Cn 608.3-.11 (Mast von Schweinen), Vn 20.3-.11 (Weindistribution) und Jn 829.4-.19 (Bronzelieferungen) passen, in denen neben anderen dieselben Ortsnamen verzeichnet sind: pi-*82, me-ta-pa, pe-to-no, pa-ki-ja-si, a-pu -we, $^{a}$-ke-re-wa, ro-u-so/e-ra-to, ka-ra-do-ro, ri-jo in der Diesseitigen Provinz; ti-mi-to-a-ke-e, ra-wa-ra-ta $a_{2}$, sa-ma-ra, a-si-jati-ja, e-ra-te-re-wa, za-ma-e-wi-ja, *e-ro in der Jenseitigen Provinz. ${ }^{292}$ Für die pylische Cn-Serie ist zu sagen, dass die Registrierung von wollproduzierenden Schafen und Ziegen auch in Bezirken der Jenseitigen Provinz stattgefunden haben müsste. So lässt die Aufzeichnung von Tieren in ti-mi-to a-ke-e (PY Cn 600.7.8.11-.15) vermuten, dass es für diesen Bezirk ebenfalls eine we-re-ke-Tafel gegeben haben könnte. ${ }^{293}$ Vielleicht ist davon auszugehen, dass die Angaben über die Tierhaltung in den einzelnen Gemeinden der Jenseitigen Provinz nur deshalb so spärlich ausfallen, weil bei der Zerstörung des Palastes noch nicht alle Informationen eingelangt waren.

Wenn die we-re-ke-Tafeln Aufzeichnungen von wollproduzierenden Schafen und Ziegen des pylischen Reiches sind, stand dem Palast jedoch nur eine geringe Anzahl von Tieren und damit verbunden wenig Wolle für die Textilwerkstätten zur Verfügung (Tab. 13).

Auch wenn wir die lückenhaften Angaben mancher Texte berücksichtigen und annehmen, dass nicht alle Aufzeichnungen im Stile der we-re-ke-Tafeln vorhanden und die erhaltenen Aufzeichnungen oft lückenhaft sind, bleibt die

${ }^{292}$ Vgl. Ilievski 1968, 631.

${ }^{293}$ Ist ein Zusammenhang mit $r a-w a-r a-t a_{2}$ gegeben, einem Bezirk der Jenseitigen Provinz? Tatsächlich könnte dieser Bezug geben sein (Bennet 1998, 124f.) und wir hätten damit einen indirekten Beleg für Aufzeichnungen von Tieren im Einzugsgebiet von ra-wa-ra- $\mathrm{ta}_{2}$. 
Gesamtzahl der wollproduzierenden Tiere gering. Auf den Umstand, dass für Pylos weit weniger Tiere (12500) verzeichnet sind als für Knossos (100000) wies bereits ST. HILLER hin. ${ }^{294}$

Zunächst ist, wie aus den vorhergehenden Ausführungen ersichtlich, ${ }^{295}$ anzumerken, dass nicht alle Schafe und Ziegen der pylischen Cn-Serie dem Palast für die Wollproduktion zur Verfügung standen, sondern nur jene, die in den we-re-ke-Texten erfasst wurden. Dies verringert natürlich die Anzahl der wollproduzierenden Tiere zusätzlich. Nachdem für Knossos ca. 100000 Schafe (ohne Ziegen!) als Wolllieferanten geschätzt sind, ${ }^{296}$ kann es sich bei den Schafen und Ziegen der we-re-ke-Tafeln nicht um den gesamten Bestand an Woll- und Haarlieferanten des Reiches von Pylos gehandelt haben. Es muss also eine andere Lösung gefunden werden.

\begin{tabular}{|c|c|c|c|c|}
\hline Ort & OVIS $^{\mathrm{m}}$ & ovis $^{\mathrm{f}}$ & $\mathrm{CAP}^{\mathrm{m}}$ & $\mathrm{CAP}^{\mathrm{f}}$ \\
\hline $\begin{array}{l}p i-* 82 \\
\text { (Cn 131) }\end{array}$ & 720 & & & 54 \\
\hline $\begin{array}{l}\text { ma-ro-pi } \\
\text { (Cn 131) }\end{array}$ & 1137 & 135 & & 55 \\
\hline $\begin{array}{l}\text { ro-u-so } \\
\text { (Cn 285) }\end{array}$ & 366 & & 206 & 20 \\
\hline $\begin{array}{l}\text { ro-u-so a-ka-na-jo } \\
\text { (Cn 328) }\end{array}$ & 450 & 190 & 160 & 60 \\
\hline $\begin{array}{l}? \\
(\text { Cn 436) }\end{array}$ & 100 & 150 & 38 & \\
\hline $\begin{array}{l}? \\
(\operatorname{Cn} 441+\operatorname{Cn} 1069)\end{array}$ & & & & 10 \\
\hline $\begin{array}{l}? \\
(\mathrm{Cn} 491+\mathrm{Xn} 1445)\end{array}$ & 60 & 50 & 12 (CAP) & 26 \\
\hline Summe & 2833 & 525 & 416 & 225 \\
\hline
\end{tabular}

Tab. 13: Anzahl der we-re-ke-Schafe in der pylischen Cn-Serie.

Die Tiere der Texte PY Cn 131, Cn 202, Cn 285, Cn 328, Cn 436, Cn 441, Cn 491 und Cn 1197 wurden in den Pferchen (we-re-ke) der jeweiligen Bezirke registriert. Eine solche Registratur wurde in klassischer Zeit im Frühjahr durchgeführt, als die Schafe geschoren wurden. ${ }^{297}$ Gleichzeitig fand eine Trennung in „Herren- und Knechtvieh“ statt, bevor die Tiere aus den Ställen auf die Weiden entlassen wurden. ${ }^{298}$ Des Gleichen wissen wir, dass Jungtiere nach der Geburt zu deren Schutz zunächst in eigenen Ställen oder Pferchen gehalten wurden, und erst ab einem späteren Zeitpunkt (nach einem Jahr) den Herden

${ }^{294}$ Hiller-Panagl 1976, 139f: „Die Anzahl der auf Kreta registrierten Schafe liegt bei ca. 100000 - die der pylischen Kleintiere bei insgesamt ca. 12500. Davon entfallen - nach Ilievski - auf männliche Schafe ca. 8200, auf weibliche etwa 1500, auf männliche Ziegen ca. 1000 und weibliche Ziegen etwa 750. - Allerdings wissen wir nicht, wieviel vom ursprünglichen Tafelmaterial uns heute verloren ist; dafür, dass der Verlust vielleicht höher, als gewöhnlich angenommen wird, anzusetzen ist, könnten die folgenden Überlegungen von L. R. Palmer sprechen: L. R. Palmer zeigt, dass von den 16 pylischen Bezirken der von pi-82, welcher uns am ausführlichsten überliefert ist, folgenden Tierbestand aufweist: Unter insgesamt 5500 Tieren befinden sich ca. 4500 Schafe, wobei es durch die geringe Anzahl der weiblichen Schafe von nur 475 wahrscheinlich wird, dass auch hier nicht der gesamte Viehbestand erfaßt ist“.

295 Zu den nicht zur Verfügung stehenden Tieren s. S. 68f., 42ff., 47ff.

296 Killen 1993, 209; Hiller-Panagl 1976, 130; Halstead 1990-91, 343.

${ }^{297}$ Ilievski 1968, 621 u. Fn. 29; Orth 1921, 387.

298 Magerstedt 1859, 134. 
eingegliedert wurden. ${ }^{299}$ Dies erfolgte also zu dem Zeitpunkt als sie bereits kräftig genug waren mit den älteren Tieren in den Herden gehalten zu werden und es möglich war sie für die Wollproduktion heranzuziehen. ${ }^{300}$

Wäre es nicht vorstellbar in den Schafen und Ziegen der we-re-ke-Texte solche Jungtiere zu sehen? Es könnte sich demnach um Schafe und Ziegen gehandelt haben, die zum Zeitpunkt der Aufzeichnung der Texte das Alter erreicht hatten, um aus den Pferchen ausgegliedert und den wollproduzierenden Herden zugeführt zu werden. ${ }^{301}$ Wenn von einem 1:1 Verhältnis der Geburt von weiblichen und männlichen Jungtieren ausgegangen wird, ${ }^{302}$ fehlen allerdings viele weibliche Schafe in den Aufzeichnungen. Doch waren diese wahrscheinlich nicht alleine für die Wollproduktion, sondern auch für die Zucht vorgesehen, und wurden deshalb womöglich in anderen (nicht erhaltenen) Aufzeichnungen erfasst. $^{303}$ Die we-re-ke-Tafeln verzeichnen also nur die Schafe und Ziegen, die als Jungtiere neu in die Herden eingegliedert wurden und Wolle und Haare liefern konnten. ${ }^{304}$ Dabei wurden anscheinend auch einige weibliche Tiere erfasst, die wohl als nicht für die Zucht geeignet erachtet wurden und somit ausschließlich als Wolllieferanten dienten. Wenn wir von dieser Interpretation ausgehen, lassen sich für Schafe einzelner „Hirten“ Hochrechnungen anstellen, die auf die tatsächlichen Größen der Herden rückschließen lassen. PY Cn 131.2 verzeichnet für den „Hirten“ pi-me-ta 200 männliche Schafe. Geht man von einem 1:1 Verhältnis an männlichen und weiblichen Geburten aus, so müssen wir mit insgesamt 400 Jungtieren rechnen. Bei einer angenommenen Zuwachsrate von $80 \%$, sind dafür 500 Mutterschafe vorauszusetzten. ${ }^{305}$ Somit kommen wir auf eine Anzahl von 900 Schafen in der Verantwortung des pi-me-ta. Hinzuzu-

299 Varro rust.2,2,15; Orth 1921, 385.

300 Vgl. Ryder 1993, 17.

301 Vgl. Killen 1993, 214f.; Halstead 1990-91, 356.

${ }^{302}$ Halstead 1990-91, 345.

${ }^{303}$ Weibliche Schafe, die trächtig waren oder säugten, wurden getrennt von den sonstigen Herdentieren gehalten; s. Orth 1921, 385. Die KN Da-Dg-Serie verzeichnet männliche und weibliche Schafe, für die nach Ausweis der KN Dk(2)-Serie dieselbe Wollleistung vorausgesetzt wird. Demgegenüber steht die KN Dl-Serie, die Muttertiere verzeichnet, die weniger Wolle lieferten (Halstead 1990-91, 345ff.). Könnten die weiblichen Schafe der PY CnSerie, diejenigen weiblichen Tiere repräsentieren, die in Aufzeichnungen vergleichbar zu den knossischen Da-Dg-Serien erfasst wurden? Finden sich demgegenüber die Muttertiere der knossischen Dl- und Do-Serien nicht in den Texten der pylischen Cn-Serie?

${ }^{304}$ Sie sind wohl als die Tiere zu sehen, die in den knossischen D-Serien als ki und ne ki bezeichnet werden. Es mussten also Tiere gewesen sein, die älter als ein Jahr waren; vgl. van de Mieroop 1993, 163: „The aš-ur 4 - animals therefore must have been more than a year old, probably some 16-17 months, when they were ready for their first plucking. Their fleece must have been longer than that of animals that had been plucked the previous year, which probably accounts for the fact that they are mentioned separately in the texts". Da die pylische Cn-Serie nicht den Wollertrag wiedergibt, sondern den Zuwachs an Schafen und Ziegen, war in dieser Serie auch die Kennzeichnung der Jungtiere als ki oder ne ki nicht nötig. In der klassischen Zeit wurden vor allem in Kleinasien die Haare von Ziegen zur Filzfabrikation verwendet (Keller 1909, 302). So sollte das Auftauchen von Ziegen in der pylischen Cn-Serie nicht verwundern, denn möglicherweise nutzten bereits die Mykener Ziegen als Haarlieferanten.

305 Die angenommene Zuwachsrate von 80 \% bezieht sich auf Angaben zur Tierzucht in Mesopotamien (Renger 2000, 163f.; van de Mieroop 1993, 163); ein Verhältnis von 1:1 an Muttertieren zu Jungtieren, wie in den knossischen Da-Dg-Serien angegeben, scheint unwahrscheinlich zu sein; dazu Halstead 1990-91, 345: „the average lambing rate is unlikely to have been as high as $100 \%$; a significant number of lambs are likely to have died before transfer to the wool flocks; and a significant number of wool sheep may have died or been culled before 5-6 years of age.“ 
rechnen sind dann weiters die weiblichen Tiere, welche keine Jungen warfen, die älteren Hammel und die Eber. Rein hypothetisch sind die nun folgenden Berechnungen zu betrachten, da uns keine Zahlenangaben zur Verfügung stehen, die einen schlüssigen Anhaltspunkt liefern könnten. Bliebe das Verhältnis von weiblichen zu männlichen Tieren relativ konstant bei $1: 1$ so kämen zu den Mutterschafen noch ca. 500 ältere männliche Schafe (Hammel und Eber) hinzu, womit pi-me-ta für eine Gesamtzahl von ca. 1400 Schafen (500 Muttertiere, 500 Hammel und Eber, 400 Jungtiere) verantwortlich gewesen wäre. Allerdings müssten dann wieder diejenigen Tiere abgezogen werden, die für die Wollproduktion zu alt ( pa-ra-jo) wurden und ausgesondert gehörten. Nehmen wir an, dass genausoviele Jungtiere hinzukamen, wie alte Schafe ausgesondert wurden, müssten wir 400 Stück abziehen. Somit würde sich eine Gesamtzahl von 1000 Tieren in der Verantwortung des pi-me-ta ergeben. Doch wie gesagt, sollten diese Berechnungen als rein hypothetisch betrachtet werden, da viele Faktoren unsicher sind: in welchem Verhältnis wurden männliche und weibliche Tiere gehalten, wieviele Tiere wurden frühzeitig geschlachtet, wurden mehr oder weniger Jungtiere als Ersatz für die alten Schafe eingegliedert, wie hoch war die Zuwachsrate tatsächlich, welcher Prozentsatz wurde an die „Collectors“ abgegeben? Als gesichert kann nur gelten, dass wir mit einer weit höheren Anzahl an Schafen und Ziegen als Woll- und Haarlieferanten rechnen dürfen als die erhaltenen we-re-ke-Texte verzeichnen.

Auch die Schafe und Ziegen der we-re-ke-Texte waren der Verantwortung von „Hirten“ übergeben. Doch während die knossischen D-Serien die wollproduzierenden Schafe und deren Wollertrag verzeichneten, registrierten die were-ke-Texte der pylischen Cn-Serie Jungtiere von Schafen und Ziegen, die in die Herden eingegliedert wurden. Dabei stand nicht der Wollertrag im Vordergrund - dieser ist in den Texten auch niemals aufgelistet - sondern die Anzahl der Jungtiere, die ab diesem Moment Wolle liefern konnten. Die Aufzucht von jungen Schafen und Ziegen in den Bezirken verwundert nicht, wenn man bedenkt, dass in denselben Orten - nachweislich in zwei, $a$-si-ja-ti-ja und e-rate-re-wa-pi, - auch Schafe gehalten wurden, die für die Zucht gedacht waren (ovIS + TA). Desgleichen finden sich in der Cn-Serie Texte, die Schafe nennen, die aus dem Produktionprozess ausgegliedert wurden, da sie für die Wollproduktion zu alt waren ( $p a-r a-j o$ ovIS $\left.{ }^{\mathrm{m}}\right)$. Daneben sind solche Schafe eingetragen, die vielleicht als Wolllieferanten nicht in Frage kamen, da deren Fell dunkel gefärbt war (wo-ne-we ovIS ${ }^{\mathrm{m}}$ ) und deshalb als Jungtiere ausgesondert wurden. Auf denselben Tafeln sind Aufzeichnungen vorhanden, die belegen, dass Schafe, Ziegen und Schweine an „Collectors“ und „Hirten“ vergeben wurden, sei es als Versorgungsgüter oder aufgrund eines Überschusses an Nachwuchs. Auffällig ist, dass vor allem die „Collectors“ eine beträchtliche Anzahl an weiblichen Schafen und Ziegen erhielten. ${ }^{306}$ Möglicherweise fiel es dem Palast leichter, sich von diesen zu trennen als von den Hammeln, die bessere Wolle lieferten. Weibliche Tiere geben jedoch Milch und konnten für die Zucht verwendet werden, was wiederum von den „Collectors“ gewünscht sein konnte. Nicht zu sagen ist, ob es sich bei diesen Tieren ebenfalls um Jungtiere gehandelt hat, oder ob ältere Schafe und Ziegen vergeben wurden. Ich würde jedoch zur Vergabe von Jungtieren tendieren, da sich der Palast wohl kaum von bereits in der Wollproduktion und Zucht stehenden Tieren getrennt haben wird. Die pylische $\mathbf{C n}$-Serie wäre also eine Bestandsaufnahme des Neuzuganges und des Abganges von Tieren in die palatialen Herden beziehungsweise aus diesen heraus.

${ }^{306}$ Vgl. Halstead 1990-91, 358 u. Tab. 6. 
Die Schafe, Ziegen und Schweine der PY Cn-Serie waren allesamt unter der Verantwortung von „Hirten“. Die Aufteilung der Herden an verschiedenen Orten stellt kein Problem dar, wenn man bedenkt, dass die Tiere nicht von diesen „Hirten“ gehütet wurden, sondern von Hirten im eigentlichen Sinne. ${ }^{307}$ Die Ortsangaben der we-re-ke-Texte beziehen sich anscheinend auf die Aufzuchtorte der jungen Schafe und Ziegen. Die Toponyme der weiteren Cn-Tafeln (außer Cn 4 und Cn 595) könnten den jeweiligen „Registrierungsort“ der Tiere angeben, ohne dass diese auch tatsächlich das ganze Jahr über dort gewesen sein mussten. ${ }^{308}$ Wenn man bedenkt, dass die Verteilung von Herden jeweils im Frühjahr erfolgte, ${ }^{309}$ könnten in den genannten Orten die Winterquartiere der Tiere gewesen sein. Dies würde jedoch für die Vergabe von älteren Schafen und Ziegen an die „Collectors“ sprechen, da die Texte, auf denen solche Herden erfasst wurden, nicht unter die Kategorie der we-re-ke-Texte fallen. Doch wären demzufolge auch ältere Tiere den „Hirten“ überlassen worden. Vielleicht sollten wir davon ausgehen, dass die Schafe, Ziegen und Schweine an den Orten gezüchtet wurden. Dann müssen die auf den we-re-ke-Texten erfassten Schafe und Ziegen Jungtiere sein, die in die Schafhürden/Pferche kamen, wobei man ihre Anzahl registrierte. Es wären dann immer noch junge Schafe und Ziegen, die den palatialen Herden zugeführt wurden, jedoch nicht aus den, sondern in die Pferche. Ein Pferch wäre demnach der erste Anlaufpunkt von Jungtieren gewesen, der Ort der Registierung und damit wohl der Platz der Schafschur und vielleicht das Winterquartier aller palatialen Schaf- und Ziegenherden. ${ }^{310}$ Damit müssen wir nicht nur für die großen Bezirke we-reke-Texte annehmen, sondern auch für weitere (kleinere) Gemeinden. ${ }^{311}$ Darauf könnten die Tafeln mit den beiden Ortsnamen ma-ro-pi (Cn 131.6) und ro-u-so $a-k a-n a-j o$ (Cn 328) hindeuten. Der Palast von Pylos hatte also Winterlager (we-re-ke = palatiale Pferche) für seine Herden, die über das ganze pylische Reich verteilt waren. Gleichzeitig waren diese auch die Registrierungsorte für die Wollproduktion neu hinzukommender Jungtiere. ${ }^{312}$ Diese könnten aus den Zuchtstallungen (ta-to-mo) bei Erreichen des für die Wollproduktion geeigneten Alters an die palatialen Pferche gesandt worden sein. Tiere, die der Palast an die „Collectors“ und „Hirten“ vergab, stammten vermutlich ebenfalls aus denselben Zuchtstallungen. All diese Schafe, Ziegen und Schweine wurden an die jeweiligen Registierungsorte, wo sich die palatialen Pferche befanden, gesandt; da sie der Palast jedoch an die „Collectors“ und „Hirten“ abgab, wurden auf den Texten zwar dieselben Ortsnamen verzeichnet wie auf den we-re-keTexten, doch ohne dem Vermerk we-re-ke, der ausdrückt, dass die Tiere den palasteigenen Herden in den palatialen Pferchen eingegliedert wurden. ${ }^{313}$

307 Siehe S. 54, 65f.

308 Zur Transhumanz s. Ilievski 1968, 627f., Doria 1958, 59; ferner s. Deger-Jalkotzy 1988, 119.

309 Vgl. Orth 1921, 391 für die klassische Zeit; Ryder 1993, 15 und Postgate 1992, 160 für Mesopotamien.

310 Bereits M. Doria vermutete unter we-re-ke Winterlager für die Schafe und Ziegen (località di pianura?); s. Doria 1958, 59. Ilievski 1968, 627f., der jedoch die Pferche (we-re-ke) als „summer enclosures“ betrachtete; s. ferner Deger-Jalkotzy 1988, 119.

311 Vgl. Killen 1993, 215.

312 Auch in Mesopotamien wurden die Jungtiere anscheinend erst bei der ersten Schur erfasst; s. van de Mieroop 1993, 163.

313 Weibliche Schafe und Ziegen, die nicht nur für die Wollproduktion sondern auch in der Zucht eingesetzt wurden, müssen anderswo verzeichnet worden sein. Auch in den Dokumenten aus Uruk wurden hauptsächlich männliche Jungtiere als Neuzugänge erfasst; s. van Driel 1993, 234f. 
PY
Ce 660
f.a a-ke-o, a-ke-re
me-ta-pa, pa-ro ka-ra-su-no CAP $^{\mathrm{m}} 30$

$\mathrm{S} 4-\mathrm{H} 21$

PY

Ce 665

ne-wo-pe-o , po-ti-ni-ja ovis ${ }^{\mathrm{m}} 100$ sus 190

$\mathrm{S} 4-\mathrm{H} 21$

PY

1285 ma-se-de, ovis ${ }^{m}$ 6[

$\mathrm{S} 4-\mathrm{H} 21$

Die pylische Cc-Serie enthält nur sechs Texte, wobei drei (PY Cc 1258, Cc 1283, Cc 1284) fragmentarisch erhalten sind und sich somit jeder eindeutigen Interpretation entziehen. Die Tafel PY Cc 660 lässt sich dagegen mit den Texten der pylischen Cn-Serie vergleichen, welche den Ausdruck a-ko-ra aufweisen. Der einzige Unterschied besteht darin, dass auf Cc $\mathbf{6 6 0}$ anstelle von $a-k o-r a$ das Verb $a$-ke-re Verwendung fand. Trotzdem dürfen wir annehmen, dass die 30 angeführten Ziegen vom pylischen Palast an $a-k e-o$ abgegeben wurden.

Auch im Falle der zwei weiteren vollständiger erhaltenen Texte der pylischen Cc-Serie kann davon ausgegangen werden, dass die Tiere vom Palast vergeben wurden. So sind die 100 Schafe und die 190 Schweine der Tafel PY Cc 665 offensichtlich an die Göttin Potnia respektive deren Heiligtum in ne-wo-pe-o gesandt worden. Der enge Kontakt zwischen dem Palast als Herdenbesitzer und Gottheiten (Hermes und Potnia) als Empfänger von Tieren zeigt sich auch in den knossischen Texten, wo ebenfalls Tiere aus dem Palastbestand an Hermes (KN D 411) beziehungsweise Potnia (KN Dl-Serie) überstellt wurden. ${ }^{314}$ Allerdings könnten die Aufzeichnungen der Texte von PY Cc 665 und KN D 411 dahingehend gedeutet werden, dass die Tiere vom Palast an die Gottheiten abgegeben wurden und somit nicht als Wolllieferanten dienten. Die Herden der Dl-Serie, welche zur Göttin Potnia gehörten, dagegen scheinen weiterhin vom knossischen Palast verwaltet worden zu sein, was durch die Aufzeichnung des Wollertrages zu erkennen ist. ${ }^{315}$

Auch die Tafel PY Cc 1285 kann dahingehend gedeutet werden, dass die (mindestens) sechs männlichen Schafe vom Palast abgegeben wurden. Dies aufgrund der Tatsache, dass das Wort ma-se-de entweder den Allativ eines Toponyms ma-se (nach $\mathrm{ma}^{-}{ }^{\circ}$ ) angibt oder aber einen Personennamen im Dativ (für $\left.m a-^{\circ}\right) .{ }^{316}$

\section{Literaturverzeichnis}

Acta Mycenaea II: Communications (Minos XII). Proceedings of the fifth International Colloquium on Mycenaean Studies, held in Salamanca, 30 March-3 April 1970. Actes du cinquieme colloque internationale des études myceniénnes, tenu à Salamanque 30 Mars3 Avril 1970 (Hrsg. Martín Ruipérez). Salamanca 1972.

Administrative Documents in the Aegean and Their Near Eastern Counterparts. Proceedings of the International Colloquium, Naples, February 29-March 2, 1996 (Hrsg. Massimo Perna). Turin 2000.

314 Siehe S. 59; vgl. Weilhartner 2002, 150; Nosch 2000, 211ff.

315 Siehe S. 59f.

${ }^{316}$ Vgl. Weilhartner 2002, 151, der für ma-se ein Heiligtum annimmt, zu dem die Lieferung der Schafe gelangen sollte. Shelmerdine 1985, 76 dagegen spricht sich für einen Personennamen aus. 
A-na-qo-ta (= Minos 33-34 [1998-1999]) presented to John Killen (Hrsg. John Bennet). Salamanca 2001.

Aegean Seals, Sealings and Administration (= Aegaeum 5). Proceedings of the NEH-Dickson Conference of the Program in Aegean Scripts and Prehistory of the Department of Classics, University of Texas at Austin, January 11-13, 1989 (Hrsg. Thomas G. Palaima). Liège 1990.

Atti e memorie del $1^{\circ}$ congresso internazionale di Micenologia. 1 Archeologia, 2 Epigrafia Micenea, 3 Storia. Roma 27 Settembre-30 Ottobre 1967 (Hrsg. Carlo Gallavotti). Rom 1968.

Atti e memorie del secondo congresso internazionale di Micenologia. Vol. I Filologia, Vol. II Storia, Vol. III Archaeologia. Roma-Napoli, 14-20 ottobre 1991 (Hrsg. Ernesto De Miro, Louis Godart, Anna Sacconi). Rom 1996.

Colloquium Mycenaeum. Actes du sixième colloque international sur les textes Mycéniens et Égéens tenu à Chaumont sur Neuchâtel du 7 au 13 Septembre 1975 (Hrsg. Ernst Risch und Hugo Müllenstein). Neuchâtel 1979.

Die Indogermanen und das Pferd. Akten des Internationalen interdisziplinären Kolloquiums Freie Universität Berlin, 1.-3. Juli 1992. (Hrsg. B. Hänsel und St. Zimmer). Festschrift für Bernfried Schlerath. Budapest 1994.

Domestic Animals of Mesopotamia. Part I (= BSA. Bulletin on Sumerian Agriculture 7. Hrsg. J. Nicholas Postgate und Marvin A. Powell). Cambridge 1993.

Economy and Politics in the Mycenaean Palace States. Proceedings of a Conference held on 1-3 July 1999 in the Faculty of Classics, Cambridge (Hrsg. Sofia Voutsaki and John Killen). Cambridge 2001.

Floreant Studia Mycenaea I und II. Akten des X. Internationalen Mykenologischen Colloquiums in Salzburg vom 1.-5. Mai 1995 (Hrsg. Sigrid Deger-Jalkotzy, Stefan Hiller, Oswald Panagl), Bd. 1 und Bd. 2. Wien 1999.

Interdependency of Institutions and Private Entrepreneurs (MOS Studies 2). Proccedings of the second MOS Symposium (Leiden 1998) (Hrsg. A.C.V.M. Bongenaar). Leiden 2000.

Mykenaïka (= BCH Suppl. 25). Actes du IXe Colloque international sur les textes mycéniens et égéens organisé par le Centre de l’Antiquité Grecque et Romaine de la Fondation Hellénique des recherches Scientifiques et l'Ècole francais d'Athénes, 2-6 octobre 1990 (Hrsg. Jean-Pierre Olivier). Paris 1992.

Österreichische Forschungen zur ägäischen Bronzezeit. Akten der Tagung am Institut für Klassische Archäologie der Universität Wien, 2.-3. Mai 1998 (Hrsg. Fritz Blakolmer). Wien 2000.

Politeia I (= Aegaeum 12). Society and State in the Aegean Bronze Age. Proceedings of the 5th International Aegean Conference/5e Rencontre égéenne internationale. University of Heidelberg, Archäologisches Institut 10-13 April 1994 (Hrsg. Robert Laffineur und WolfDietrich Niemeier). Liège 1995.

Proceedings of the Cambridge Colloquium on Mycenaean Studies (Hrsg. Leonard R. Palmer und John Chadwick). Cambridge 1966.

Sandy Pylos. An Archaeological History from Nestor to Navarino (Hrsg. Jack L. Davis). Austin 1998.

Society and Economy in the eastern Mediterranean (c. 1500-1000 B.C.). Proceedings of the International Syposium held at the University of Haifa from the $28^{\text {th }}$ of April to the $2^{\text {nd }}$ of May 1985 (Hrsg. Michael Heltzer und Edward Lipinski). Leuven 1988.

Studia Mycenaea 1988 (= ŽA. Monographies No 7). Skopje 1989.

Studies in Greek, Italic, and Indo-European Linguistics. Offered to Leonard R. Palmer on the Occasion of His Seventieth Birthday. Innsbrucker Beiträge zur Sprachwissenschaft 16 (Hrsg. Anna Morpurgo-Davies und Wolfgang Meid). Innsbruck 1976.

TEXNH I und II (= Aegaeum 16). Craftsmen, Craftswomen and Craftsmanship in the Aegean Bronze Age. Proceedings of the 6th International Aegean Conference/6e Rencontre égéenne internationale. Philadelphia, Temple University, 18-21 April 1996. (Hrsg. Robert Laffineur und Philip P. Betancourt). Liège 1997.

Texts, Tablets and Scribes (= Suplementos a Minos Núm. 10). Studies in Mycenaean Epigraphy and Economy. Offered to Emmett L. Bennett Jr. (Hrsg. J.-P. Olivier und Th. G. Palaima). Salamanca 1988. 
The Role of the Ruler (= Aegaeum 11). Proceedings of a Panel Discussion presented at the Annual Meeting of the Archaeological Institute of America. New Orleans, Louisiana, 28 December 1992 (Hrsg. Paul Rehak). Liège 1995.

Tractata Mycenaea. Proceedings of the Eighth International Colloquium on Mycenaean Studies, held in Ohrid, 15-20 September 1985 (Hrsg. Petar Hr. Ilievski und Ljiljana Crepajac). Skopje 1987.

\section{Aravantinos, Vassilis:}

1987 The Mycenaean Inscribed Sealings from Thebes: Preliminary Notes. In: Tractata Mycenaea. Skopje 1987, 13-27.

1990 The Mycenaean Inscribed Sealings from Thebes: Problems of Content and Function. In: Aegean Seals, Sealings and Administration. Liège 1990, 149-174.

Aura Jorro, Franciscos:

1985 Diccionario Griego - Espanol I. Dicionario Micénico I. Madrid 1985.

1993 Diccionario Griego - Espanol II. Dicionario Micénico II. Madrid 1993.

Barth, Rudolf (Hrsg.):

1953 Brehms Tierleben. Salzburg 1953.

Bartoněk, Antonin:

2003 Handbuch des mykenischen Griechisch. Heidelberg 2003.

Benecke, Norbert:

1994 Der Mensch und seine Haustiere. Die Geschichte einer jahrtausendealten Beziehung. Stuttgart 1994.

Bennet, John:

1992 «Collectors» or «owners»? An examination of their possible functions within the palatial economy of LM III Crete. In: Mykenaïka. Paris 1992, 65-101.

1998 The Linear B Archives and the Kingdom of Nestor. In: Sandy Pylos. Austin 1998, 111-133.

1999 The Mycenaean Conceptualization of Space or Pylian Geography (... yet again!). In: Floreant Studia Mycenaea I. Wien 1999, 131-157.

Bennett-Olivier $=$ Bennett, Emmett L. und Olivier, Jean-Pierre (Hrsg.):

1973 The Pylos Tablets Transcribed. Part I: Texts and Notes. Rom 1973.

1976 The Pylos Tablets Transcribed. Part II: Hands, concordances, Indices. Rom 1976.

\section{Bertelsmann Lexikon:}

1973 s.v. Mast. In: Bertelsmann Lexikon Bd. 6 (1973 [1983]), 299.

Carlier, Pierre:

1992 Les collecteurs sont-ils des fermiers? In: Mykenaïka. Paris 1992, 159-166.

Chadwick, John:

1957 Potnia. In: Minos 5 (1957), 117-129.

1976 The Mycenaean World. Cambridge 1976 (Nachdruck 1994).

1987 The Muster of the Pylian Fleet. In: Tractata Mycenaea. Skopje 1987, 75-84.

Chadwick-Godart-Killen u.a. = Chadwick, John, Godart, Louis, Killen, John T., Olivier, Jean-Pierre, Sacconi, Anna, Sakellarakis, I. A.:

1986 Corpus of Mycenaean Inscriptions from Knossos. Vol. I (1-1063). Rom 1986.

1990 Corpus of Mycenaean Inscriptions from Knossos. Vol. II (1064-4495). Rom 1990.

1997 Corpus of Mycenaean Inscriptions from Knossos. Vol. III (5000-7999). Pisa, Rom 1997.

1998 Corpus of Mycenaean Inscriptions from Knossos. Vol. IV (8000-9947) and Index to Volumes I-IV. Pisa, Rom 1998.

Chantraine, Pierre:

1984 Dictionnaire étymologique de la langue grecque 1-2, 3-4. Histoire des mots. Paris 1984

Deger-Jalkotzy, Sigrid:

1978 E-QE-TA. Zur Rolle des Gefolgschaftswesens in der Sozialstruktur mykenischer Reiche. Wien 1978.

1988 Noch einmal zur Ea-Serie von Pylos. In: Texts, Tablets and Scribes. Salamanca 1988, 97-122.

2001 Working for the Palace: Some Observations on PY An 261. In: A-na-qo-ta, 65-81. 


\section{Dickinson, Oliver:}

1994 The Aegean Bronze Age. Cambridge 1994.

Doria, Mario:

1958 Interpretazioni di testi micenei II, Le inscrizioni della classe Cn di Pilo. Triest 1958.

Driessen, Jan:

1992 «Collector’s items». Observations sur l'élite mycénienne de Cnossos. In: Mykenaïka. Paris 1992,197-214.

van Driel, Govert:

1993 Neo-Babylonian Sheep and Goats. In: Domestic Animals of Mesopotamia. Part I. Cambridge 1993, 219-258.

Frisk, Hjalmar:

1991 Griechisches Etymologisches Wörterbuch. Bd. I: A-KO (1973), Bd. II: K $\rho-\omega$ (1991).

Gillis, Carole:

1997 The Smith in the Late Bronze Age - State Employee, Independent Artisan, or Both? In: TEXNH II. Liège 1997, 505-513.

\section{Godart, Louis:}

1971a Les tablettes de la série Co de Cnossos. In: Acta Mycenaea II (1971), 418-424.

1971b Valeur des idéogrammes dans les tablettes de Cnossos. In: KrChron 23, 1 (1971), 89-94.

1992 Les collecteurs dans le monde égéen. In: Mykenaïka. Paris 1992, 257-283.

1999 Les sacrifices d'animaux dans les textes mycéniens. In: Floreant Studia Mycenaea I. Wien 1999, 249-256.

Gruppe, Otto:

1906 Griechische Mythologie und Religionsgeschichte. In: HbAW 5, 2, I (1906).

\section{Halstead, Paul:}

1990-91 Lost Sheep? On the Linear B Evidence for breeding Flocks at Mycenaean Knossos and Pylos. In: Minos 25-26 (1990-1991), 343-365.

Hiller, Stefan:

1982 Tempelwirtschaft im mykenischen Griechenland. In: AfO Beiheft 19 (1982), 94-103.

Hiller-Panagl = Hiller, Stefan und Panagl, Oswald:

1976 Die frühgriechischen Texte aus mykenischer Zeit. Zur Erforschung der Linear BTafeln. Darmstadt 1976.

\section{Hörning, Bernhard:}

1992 Artgemäße Schweinehaltung. Grundlagen und Beispiele aus der Praxis. In: Schriftenreihe der Stiftung Ökologie und Landbau. Karlsruhe 1992.

Hutton, William F.:

1990-91 The meaning of qe-te-o in Linear B. In: Minos 25-26 (1990-1991), 105-131.

Ilievski, Petar Hr.:

1968 A Re-examination of the PY Cn Tablets. In: Atti e memorie. Bd. 2. Rom 1968, 616-632.

1992 Observations on the Personal Names from the Knossos D Tablets. In: Mykenaïka. Paris 1992, 321-349.

1999 Interpretation of some Mycenaean Personal Names: Nomina theophora. In: Floreant Studia Mycenaea I. Wien 1999, 299-311.

Keller, Otto:

1909 Die antike Tierwelt. 1. Bd.: Säugetiere. Leipzig 1909 (Nachdruck Hildesheim 1963).

Killen, John T.:

1964 The Wool Industry of Crete in the Late Bronze Age. In: BSA 59 (1964), 1-15.

1968 Minoan woolgathering: a reply (Part I). In: Kadmos 7 (1968), 105-123.

1969 Minoan woolgathering: a reply (Part II). In: Kadmos 8 (1969), 23-38.

1976 Linear B a-ko-ra-ja/-jo. In: Studies in Greek, Italic, and Indo-European Linguistics. Innsbruck 1976, 117-125.

1979 The Knossos Ld(1) Tablets. In: Colloquium Mycenaeum. Neuchâtel 1979, 151-181. 
1992 Observations on the Thebes Sealings. In: Mykenaïka. Paris 1992, 366-380.

1992-93 The Oxen's Names on the Knossos Ch Tablets. In: Minos 27-28 (1992-1993), 101-107.

1993 Records of Sheep and Goats at Mycenaean Knossos and Pylos. In: Domestic Animals of Mesopotamia. Part I. Cambridge 1993, 209-218.

1995 Some Further Thoughts on 'Collectors’. In: Politeia I. Liège 1995, 213-226.

1996 Thebes Sealings and Knossos Tablets. In: Atti e memorie. Vol. I. Rom 1996, 71-82.

1999 Mycenaean o-pa. In: Floreant Studia Mycenaea II. Wien 1999, 325-341.

New Readings and Interpretations in the Pylos Tablets. In: Floreant Studia Mycenaea II. Wien 1999, 343-353.

Killen-Oliver $=$ Killen, John T. und Oliver, Jean-Pierre:

1966388 raccords de fragments dans les tablettes de Cnossos. In: Proceedings. Cambridge 1966, 47-92.

1968155 raccords de fragments dans les tablettes de Cnossos. In: BCH 92 (1968), $115-141$.

1989 The Knossos Tablets. Fifth Edition. Suplementos a Minos Núm. 11. Salamanca 1989.

Kraemer, Hans:

1940 s.v. Rind. In: RE Suppl. 7 (1940 [1958]), 1155-1185.

Kroll, Wilhelm:

1924 s.v. lana. In: RE 12, 1 (1924 [1963]), 594-617.

Lang, Mabel L.:

1966 Cn-flocks. In: Proceedings. Cambridge 1966, 250-259.

1988 Pylian Place-Names. In: Texts, Tablets and Scribes. Salamanca 1988, 185-212.

Leukart, Alex:

1994 Die frühgriechischen Nomina auf $-t \bar{a} s$ und $-\bar{a} s$. Untersuchungen zu ihrer Herkunft und Ausbreitung (unter Vergleich mit den Nomina auf -eús). In: Mykenische Studien Bd. 12. Wien 1994.

Lejeune, Michel:

1957 Mycénien dama/duma «intendant». In: Minos 5 (1957), 130-148.

1973 Le dossier sa-ra-pe-da du scribe 24 de Pylos. In: Minos 14, 1973, 60-76.

Liddell-Scott $=$ Liddell, Henry George und Scott, Robert:

1996 A Greek - English Lexicon. Oxford $1996^{9}$ (new Supplement added).

Lindgren, Margareta:

1973 I The People of Pylos. Prosopographical and Methodological studies in the Pylos Archives. Part I: A Prosopographical Catalogue of Individuals and Groups. Uppsala 1973.

1973 II The People of Pylos. Prosopographical and Methodological studies in the Pylos Archives. Part II: The Use of Personal Designations and Their Interpretation. Uppsala 1973.

Magerstedt, Adolph Friedrich:

1859 Die Viehzucht der Römer. Erste Abtheilung: Das Rind, das Schaf, die Ziege, der Hund. Sonders-hausen 1859 (Nachdruck Vaduz 1984).

Melena, José L.:

1975 Studies on some Mycenaean Inscriptions from Knossos dealing with Textiles (= Suplementos a Minos Núm. 5), Salamanca 1975.

1994-95 28 Joins and Quasi-joins of Fragments in the Linear B Tablets from Pylos. In: Minos 29-30 (1994-1995), 95-100.

133 Joins and Quasi-joins of Fragments in the Linear B Tablets from Pylos. In: Minos 29-30 (1994-1995), 271-288.

1996-1997 13 Joins and Quasi-joins of Fragments in the Linear B Tablets from Pylos. In: Minos 31-32 (1996-1997), 171-178.

van de Mieroop, Marc:

1993 Sheep and goat herding according to the Old Babylonien texts from Ur. In: Domestic Animals of Mesopotamia. Part I. Cambridge 1993, 161-182.

Nosch, Marie-Louise:

2000 Schafherden unter dem Namenspatronat von Potnia und Hermes in Knossos. In: Österreichische Forschungen zur Ägäischen Bronzezeit. Wien 2000, 211-215. 
Olck, Franz:

1907 s.v. Esel. In: RE 6, 1 (1907 [1958]), 626-676.

Olivier, Jean-Pierre:

1960 A propos d'une «liste» de desservants de sanctuaire dans les documents en Lineaire B de Pylos. Brüssel 1960.

1967 La série Dn de Cnossos. In: SMEA 2 (1967), 71-93.

1972 La série Dn de Cnossos reconsideree. In: Minos 13, 1 (1972), 22-28.

2001 Les “collecteurs”: leur distribution spatiale et temporelle. In: Economy and Politics in the Mycenaean Palace States. Cambridge 2001, 139-160.

Orth, Ferdinand:

1921 s.v. Schaf. In: RE 2 A, 1 (1921 [1964]), 373-399.

s.v. Schwein. In: RE 2 A, 1 (1921 [1964]), 801-815.

Panagiotopoulos, Diamantis:

2002 Untersuchungen zur mykenischen Siegelpraxis. 1. Textteil. Habil.-Schr. Salzburg 2002.

Palaima, Thomas G.:

1988 The scribes of Pylos. Rom 1988.

1989 Perspectives on the Pylos Oxen Tablets: Textual (and Archaeological) Evidence for the Use and Management of Oxen in Late Bronze Age Messenia (and Crete). In: Studia Mycenaea. Skopje 1989, 85-124.

1992 The Knossos Oxen Dossier: The Use of Oxen in Mycenaean Crete. Part I: General Background and Scribe 107. In: Mykenaïka. Paris 1992, 463-474.

1995 The Nature of the Mycenaean Wanax: Non-Indo-European Origins and Priestly Functions. In: The Role of the Ruler. Liège 1995, 119-139 u. Taf. 41-42.

2000 The Transactional Vocabulary of Mycenaean Sealings and the Mycenaean Administrative Process. In: Administrative Documents in the Aegean and Their Near Eastern Counterparts. Turin 2000, 261-273.

L. R. Palmer = Palmer, Leonard R.:

1954 M. Ventris and J. Chadwick: Evidence for Greek dialect in the Mycenaean archives. In: Gnomon 26 (1954), 65-67.

1956 Military Arrangements for the Defence of Pylos. In: Minos 4, 2 (1956), 120-145.

1957 1. The Pylos Tablets ed. Bennett. 2. The Knossos Tablets edd. Bennett, Chadwick and Ventris. In: Gnomon 29 (1957), 113-117.

Ventris and Chadwick, Documents in Mycenaean Greek. In: Gnomon 29 (1957), $561-581$.

1963 The Interpretations of Mycenaean Greek Texts. Oxford 1963.

1966 Some points for discussion. In: Proceedings. Cambridge 1966, 275-284.

1981 Forum. In: Nestor 8:1, 1501-1502.

R. Palmer = Palmer, Ruth:

1994 Wine in the Mycenaean Palace Economy. In: Aegaeum 10. Liège 1994.

1999 Perishable Goods in Mycenaean Texts. In: Floreant Studia Mycenaea II. Wien 1999, 463-485.

Panagl, Oswald:

1979 Textual Features in the Linear B Tablets. In: Colloquium Mycenaeum. Neuchâtel 1979, 313-322.

Peters, Joris:

1998 Römische Tierhaltung und Tierzucht. Eine Synthese aus archäozoologischer Untersuchung und schriftlich-bildlicher Überlieferung. Passauer Universitätsschriften zur Archäologie. Bd. 5. Rahden/Westf. 1998.

Plath, Robert:

1994 Pferd und Wagen im Mykenischen und bei Homer. In: Die Indogermanen und das Pferd. Budapest 1994, 103-114.

Piteros-Olivier-Melena $=$ Piteros, Christos, Olivier, Jean-Pierre und Melena, José L.:

1990 Les inscriptions en Linéaire B des nodules de Thèbes (1982): La fouille, les documents, les possibilités d'interprétation. In: BCH 114 (1990), 103-184.

Postgate, J. Nicholas:

1992 Early Mesopotamia. Society and economy at the dawn of history. London und New York 1992. 
System and Style in three Near Eastern Bureaucracies. In: Economy and Politics in the Mycenaean Palace States. Cambridge 2001, 181-194.

Renger, Johannes:

1998 Wirtschaft und Gesellschaft. In: Der Alte Orient. Geschichte und Kultur des alten Vorderasien (Hrsg. Barthel Hrouda). München 1998, 187-215.

2000 Das Palastgeschäft in der altbabylonischen Zeit. In: Interdependency of Institutions and Private Entrepreneurs. Leiden 2000, 154-183.

Richter, Will:

1972 s.v. Ziege. In RE 10 A (1972), 398-433.

Rougemont, Françoise:

2001 Some Thoughts on the Identification of the "Collectors" in the Linear B Tablets. In: Economy and Politics in the Mycenaean Palace States. Cambridge 2001, 129-138.

Ruijgh, Cornelis J.:

1967 Études sur la grammaire et le vocabulaire du grec mycénien. Amsterdam 1967.

1999 Fóv $\alpha \xi$ et ses dérivés dans les textes mycéniens. In: Floreant Studia Mycenaea II. Wien 1999, 521-535.

Ryder, M. L.

1993 Sheep and goat husbandry with particular reference to textile fibre and milk production. In: Domestic Animals of Mesopotamia. Part I. Cambridge 1993, 9-32.

Shelmerdine, C.:

1985 The Perfume Industry of Mycenaean Pylos. Göteborg 1985.

Steier, August:

1938 s.v. Pferd. In: RE 19, 2 (1938 [1964]), 1430-1444.

Tzedakis - Martlew = Tzedakis, Yannis und Martlew, Holley (Hrsg.):

1999 Minoans and Mycenaeans. Flavours of their Time. National Archaeological Museum, 12 July-27 November 1999. Athen 1999.

Uchitel, Alexander:

1984 On the 'military’ character of the o-ka tablets. In: Kadmos 23, 2 (1984), 136-163.

Weilhartner, Jörg:

2002 Die Linear B-Opfertexte der mykenischen Paläste auf Kreta und dem griechischen Festland. Diss. Salzburg 2002

Ventris - Chadwick $=$ Ventris, Michael und Chadwick, John:

1973 Documents in Mycenaean Greek. Cambridge 1973².

Yamagata, Naoko:

1995 Ritual offerings in Homer and in Linear B. In: SMEA 35 (1995), 57-68.

Ziehen, Ludwig:

1939 s.v. Opfer. In: RE 18, 1 (1939 [1959]), 579-627.

Colum. Lucius Junius Moderatus Columella: De re rustica libri duodecim (Zwölf Bücher über die Landwirtschaft). Hrsg. u. übersetzt von Will Richter. Bd. II. München 1982

Hom. Il Homer: Ilias. Übers. von Johann Heinrich Voss, hrsg. von Eduard Schwartz. Darmstadt 1994.

Hom. Od. Homer: Odyssee. Übers. von Johann Heinrich Voss, hrsg. von Eduard Schwartz. Darmstadt 1994.

Plin. nat. C. Plinius Secundus d. Ä.: Naturalis historia (Naturkunde).

Buch 8. Zoologie Landtiere. Hrsg. u. übers. von Rodrich König in Zusammenarbeit mit Gerhard Winkler. Darmstadt 1976.

Varro rust. Marcus Terentius Varro: Rerum rusticarum (Gespräche über die Landwirtschaft). Buch 1. hrsg. u. übers. von Dieter Flach. (Texte zur Forschung Bd. 66). Darmstatt 1996.

Buch 2. hrsg. u. übers. von Dieter Flach. (Texte zur Forschung Bd. 66). Darmstatt 1997.

Vorgelegt von w. M. Sigrid JalkotZy-Deger

in der Sitzung am 22. Juni 2007 
